\title{
Pseudocontact shifts in biomolecular NMR using paramagnetic metal tags
}

Christoph Nitsche and Gottfried Otting*

* Corresponding author

Address: Australian National University, Research School of Chemistry, Canberra ACT 2601, Australia

Tel.: +61261256507

E-mail addresses: christoph.nitsche@anu.edu.au (C. Nitsche), gottfried.otting@anu.edu.au (G. Otting)

URL: http:rsc.anu.edu.au/ go/index.html

\section{Keywords:}

Pseudocontact shifts

Paramagnetic metal tags

Lanthanides

Protein structure

Protein dynamics 


\section{Contents}

1. Scope of this review

1.1. Paramagnetic NMR of biological macromolecules

1.2. Radicals, transition metals, lanthanides

1.3. Diamagnetic reference

1.4. Paramagnetic tags

1.5. Advantages of pseudocontact shifts over PREs

1.5.1. High sensitivity

1.5.2. Suppression of intermolecular effects

1.5.3. Tolerance towards incomplete tagging yields

1.5.4. Superior distance range

1.6. Recent reviews on paramagnetic NMR

2. Pseudocontact shifts

2.1. $\chi$ tensor and $\Delta \chi$ tensor

2.2. Unique $\Delta \chi$ tensor representation

3. Paramagnetic properties of lanthanides

3.1. $\Delta \chi$ tensors associated with lanthanide ions

3.2. Paramagnetic relaxation enhancements by lanthanide ions

4. Determining $\Delta \chi$ tensors

4.1. Principles

4.2. Quality factors

4.3. Effective $\Delta \chi$ tensors arising from flexible metal tags

4.4. PCS measurements and assignment of paramagnetic NMR spectra

4.4.1. Resonance assignment using $\Delta \chi$-tensor fits

4.4.2. Resonance assignment by chemical exchange of metal ions

4.4.3. Using PCSs to assign diamagnetic NMR spectra

4.4.4. Additional aids for the assignment of paramagnetic NMR spectra

4.5. Paramagnetically induced residual dipolar couplings

4.6. Using RDCs to assess tag mobility

4.7. Software using PCSs

4.7.1. Programs for $\Delta \chi$-tensor determinations

4.7.2. Programs using PCSs for NMR resonance assignments of proteins

4.7.3. Programs using PCSs for protein structure computation 
5. Lanthanide tags for the generation of pseudocontact shifts

5.1. Lanthanide binding proteins

5.2. Lanthanide binding peptides

5.3. Synthetic tags

5.3.1. Chemical synthesis of ligands with metal tags

5.3.2. Covalent protein tags

5.3.2.1. EDTA tags and peak doubling due to chiral metal complexation

5.3.2.2. DTPA tag

5.3.2.3. TAHA tag

5.3.2.4. Cyclen tags

5.3.2.4.1. Double-arm cyclen tags

5.3.2.4.2. Single-arm cyclen tags binding to cysteine

5.3.2.4.3. Single-arm cyclen tags binding to $p$-azido-L-phenylalanine

5.3.2.5. Tags based on dipicolinic acid

5.3.2.6. Tags based on NTA

5.3.2.7. Tags based on IDA

5.3.2.8. Prediction of assisted complexation by protein carboxylates

5.3.2.9. NTA and IDA tags for ligation by click chemistry

5.3.2.10. Commercial availability

5.3.3. Non-covalently binding tags

5.3.3.1. Non-covalently binding synthetic tags

5.3.3.2. Non-covalently binding protein tags

5.4. Metal-binding unnatural amino acids

6. Generating PCSs with $\mathrm{Co}^{2+}$

6.1. $\mathrm{Co}^{2+}$ binding proteins

6.2. $\mathrm{Co}^{2+}$ complexes generating sizeable PCSs

6.3. Creating a $\mathrm{Co}^{2+}$ or $\mathrm{Mn}^{2+}$ binding site with an unnatural amino acid

7. Macromolecular structure from pseudocontact shifts generated by lanthanide and cobalt tags

7.1. 3D protein structure refinements using PCSs from lanthanides

7.2. 3D protein structure determinations using PCSs generated by lanthanide tags

7.3. Structure determinations of protein-protein complexes using PCSs from lanthanides

7.4. Lanthanide-generated PCSs for studies of dynamic proteins and protein-protein complexes 
7.4.1. PCSs of random coil peptides

7.4.2. Inter-domain PCSs in multiple-domain proteins

7.4.3. PCS studies of dynamic protein-protein complexes

7.4.4. Relaxation dispersion and CEST experiments on protein signals shifted by PCSs

7.4.5. PCSs for 3D structure determination of short-lived enzyme intermediates

7.5. Protein-small molecule interactions

7.5.1. Studies with lanthanide tags on the protein

7.5.2. Studies with lanthanide or cobalt tag on the ligand

8. Conclusion and prospects 


\section{Introduction}

\section{Scope of this review}

\subsection{Paramagnetic NMR of biological macromolecules}

Unpaired electrons endow their host molecules with paramagnetism, and the detection of associated phenomena in the NMR spectrum is commonly referred to as paramagnetic NMR spectroscopy. The magnetic moment of a free unpaired electron is about 660 times greater than that of a proton. Therefore, single unpaired electrons, which may be associated with either organic radicals or metal ions, generate very significant effects in NMR spectra, which extend over a long distance range compared to any magnetic interactions between nuclear spins. The long-range nature of the paramagnetic effects arising from unpaired electrons renders them a powerful NMR spectroscopic tool for the study of the structure and dynamics of biological macromolecules. This review concentrates on proteins, because NMR studies of paramagnetic macromolecules NMR have mostly been performed with proteins. Nonetheless, the same experiments and conclusions evidently also apply to DNA, RNA and carbohydrates as well as complexes thereof with ligand molecules. The authors are not aware of articles describing experiments to modify DNA or RNA with the intent to generate pseudocontact shifts, which are at the centre of this review.

\subsection{Radicals, transition metals, lanthanides}

Unpaired electrons are found, inter alia, in organic radicals and transition metal ions. Organic radicals that are stable in aqueous solution are rare. Nitroxide radicals are used most frequently. They stand out for their stability and their inertness towards chemical reaction with proteins and DNA. Site-specific labeling with synthetic nitroxide tags presents a powerful tool for obtaining structural restraints from paramagnetic effects, and the literature associated with nitroxide tags is rich and large. This review discusses nitroxides only in passing, focusing instead on paramagnetic metal ions, lanthanides in particular.

Lanthanide ions are of particular interest because of their varied paramagnetic effects. Together with the actinide series, they make up the inner transition metals or f-block of the periodic table. For the purpose of this review, we refer to d-block elements as 'transition metals' and to lanthanide ions as a separate group.

Historically, metallo-proteins containing transition metal ions have played a key role in confirming theoretical predictions of the long-range effects of paramagnetism in NMR spectra. Iron-containing heme proteins such as myoglobin and cytochrome $c$ were studied very early on, but the validity of the point-dipole approximation, which assumes that the 
magnetic dipole moment is located at a single point, is compromised in these systems. Transition metal ions harbour unpaired electrons in d-orbitals that engage in binding interactions with the immediate chemical environment, leading to partial delocalisation of the unpaired electron across the ligand orbitals. In this situation, a quantitative description of the paramagnetic effects necessitates quantum mechanical computations to locate the unpaired spin-density [1, 2]. The conjugated double-bond system of a heme ring is particularly effective in delocalising unpaired electron density.

This review focuses on pseudocontact shifts (PCS) generated by metal tags. Among the transition metal ions, only high-spin $\mathrm{Co}^{2+}$ is well suited for metal tags on proteins, combining fairly large PCSs with small paramagnetic relaxation enhancements (PRE) and stability of its complexes in aqueous solution. The $\mathrm{Mn}^{2+}$ ion generates very large PREs but no PCSs. The $\mathrm{Cr}^{3+}$ ion generates relatively small PCSs [3]. In the case of iron, the ligand field determines whether $\mathrm{Fe}^{2+}$ is paramagnetic or diamagnetic and whether the electron configuration in $\mathrm{Fe}^{3+}$ ions is high-spin or low-spin [4]. Depending on the ligand field, PCSs generated by high-spin $\mathrm{Fe}^{3+}$ can be moderately large [5] or exceedingly small [6]. The $\mathrm{Ru}^{3+}$ ion generates sizeable PCSs [7], but most of its complexes are unstable. For these reasons, chromium, iron and ruthenium ions have not been used in paramagnetic tags for the purpose of generating PCSs. $\mathrm{Ni}^{2+}$ and $\mathrm{Cu}^{2+}$ generate only small PCSs [8,9]. The paramagnetism and specific binding affinities of these two ions make them particularly suitable for the generation of PREs with the help of N-terminal peptide tags $[10,11]$.

Regarding PCSs, lanthanide ions are the only other metal ions that can produce effects as large as $\mathrm{Co}^{2+}$ or larger. The point-dipole approximation works well for lanthanide ions, as they harbour their unpaired electrons in f-orbitals, which do not interact as strongly with ligand orbitals as the d-orbitals of transition metals. Since lanthanides have no known function in biology, proteins with natural specific binding sites for lanthanide ions are rare. As the general chemical properties of lanthanide ions are broadly similar to those of calcium ions, calcium binding sites sometimes accept lanthanides in vitro. For example, calbindin $\mathrm{D}_{9 \mathrm{k}}$, a protein with two calcium binding sites, proved to be an excellent system for evaluating paramagnetic effects induced by lanthanide ions as, fortuitously, the $\mathrm{Ca}^{2+}$ ion of one of the two sites can readily be replaced by a lanthanide ion. Pseudocontact shift studies of this protein in the Bertini laboratory established a reference for determining the principal axes of the magnetic susceptibility tensors associated with paramagnetic lanthanide ions and the magnitude of their anisotropies [12]. 
Different lanthanide ions display different magnitudes of paramagnetic effects. Many lanthanide ions are much more paramagnetic than any transition metal ions and generate correspondingly large effects in NMR spectra. In addition, lanthanide ions are chemically very similar and their oxidation state $(3+)$ is chemically very stable, so that a lanthanide binding site on a protein or in a synthetic lanthanide chelating compound can bind any of the lanthanide ions with similar affinity, although details of lanthanide coordination can vary with the ionic radius.

\subsection{Diamagnetic reference}

An important point is the availability of molecules and metal ions that can serve as a suitable diamagnetic reference, as paramagnetic effects are always measured by comparison with a diamagnetic reference. Even small paramagnetic effects can be measured accurately by comparison with a suitable corresponding diamagnetic sample.

Among the lanthanide ions, $\mathrm{La}^{3+}$ and $\mathrm{Lu}^{3+}$ are diamagnetic, but $\mathrm{Y}^{3+}$ often is an even better diamagnetic reference, as its ionic radius is almost identical to the $\mathrm{Ho}^{3+}$ ion. Strictly speaking, yttrium is not a member of the lanthanide series. Therefore, the group of lanthanides and chemically similar elements are sometimes referred to as lanthanoids. This review does not make this distinction, referring simply to lanthanides throughout.

$\mathrm{Co}^{2+}$ generates fairly large PCSs without very large PREs, similar to the $\mathrm{Yb}^{3+}$ ion. Unfortunately, no diamagnetic transition metal ion with a $2+$ charge exists with exactly the same chemical properties. $\mathrm{Zn}^{2+}$ is commonly used as the diamagnetic reference for the $\mathrm{Co}^{2+}$ ion because of its similar ionic radius.

Similarly, $\mathrm{Mn}^{2+}$ generates very large PREs, but no diamagnetic metal ion can exactly reproduce its chemical properties. $\mathrm{Zn}^{2+}$ or $\mathrm{Mg}^{2+}$ often serve as diamagnetic references.

In the case of nitroxide ions, chemical reduction removes the paramagnetic character by producing a diamagnetic hydroxylamine group. Ascorbate is a commonly used reagent to achieve the required one-electron reduction.

\subsection{Paramagnetic tags}

Diamagnetic proteins have the advantage that they can be site-specifically tagged with a paramagnetic compound at many different sites of choice. The paramagnetic effects observed by NMR are most easily interpreted if a protein can be furnished with a single paramagnetic centre rather than several. Site-specific tagging is open to a wide range of tags and tagging strategies. Among the 20 natural amino acids, cysteine residues offer the most 
facile way of selective chemical modification without affecting any of the other amino acids, as the thiol group is highly nucleophilic and easy to engage in a disulfide bond.

At present, the MTSL tag (Fig. 1) is the paramagnetic tag most frequently used in protein NMR. It contains a chemically activated sulfur atom, which readily reacts with cysteine residues with formation of a covalent disulfide bond. The nitroxide group generates PREs without changing the chemical shifts. Reduction with ascorbate is a convenient way to eliminate the radical character of the tag and produce a diamagnetic reference.

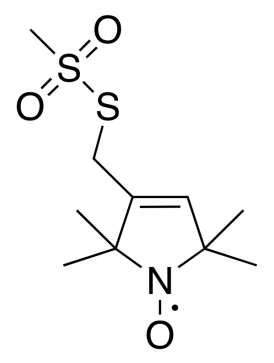

Fig. 1. Chemical structure of MTSL. The tag reacts with the thiol group of cysteine residues with formation of a mixed disulfide bond and the release of methanesulfinic acid $\left(\mathrm{CH}_{3} \mathrm{SO}_{2} \mathrm{H}\right)$.

The obvious drawback of any tag designed to react with a single cysteine residue is the need to work with a protein that contains only a single reactive thiol group. This usually requires mutation of naturally occurring cysteine residues to other amino acids and positioning of a cysteine residue at the site of interest. Often, however, mutations are found to destabilise the protein fold. In other cases, some cysteine residues may be essential for protein function.

Recently, a more general approach has been developed that uses genetically encoded amino acids containing a nitroxide group and therefore does not require the mutation of cysteine residues [13]. So far, however, the system has been established only for E. coli, where the reducing environment of the cytosol leads to chemical reduction of a large fraction of the nitroxide amino acid, resulting in a mixture of paramagnetic and diamagnetic protein.

This review focuses on pseudocontact shifts and therefore is mostly concerned with tagging approaches using paramagnetic metal ions, in particular lanthanide ions. Lanthanide ions are inert with respect to the oxidising or reducing conditions encountered under physiological conditions. All paramagnetic lanthanide ions also generate PREs. $\mathrm{Gd}^{3+}$ and $\mathrm{Mn}^{2+}$ ions are the metal ions best suited for PRE measurements, due to their large and isotropic As chelating compounds bind $\mathrm{Mn}^{2+}$ 
ions more weakly than heavier transition metal ions [14], it is much easier to produce inert complexes with $\mathrm{Gd}^{3+}$ than with $\mathrm{Mn}^{2+}$ ions.

In the case of metalloproteins, labelling with a metal tag bears the risk of exchange between metal ions in the protein and the metal ion in the tag. This situation can be addressed with tags that are kinetically inert with respect to metal exchange, such as the cyclen tags described in Section 5.3.2.4.

\subsection{Advantages of pseudocontact shifts over PRES}

\subsubsection{High sensitivity}

PCSs are simply measured as the chemical shift difference between a sample tagged with a paramagnetic metal ion and the corresponding sample tagged with a diamagnetic ion as a reference. This means that PCSs can be measured even with samples of low concentration, and in shorter time than, for example, NOEs, RDCs, scalar couplings or quantitative PREs.

\subsubsection{Suppression of intermolecular effects}

Sizeable PREs can be observed regardless of whether the geometry between paramagnetic centre and nuclear spin is fixed (as for intramolecular effects) or variable (as for non-specific intermolecular effects). This can make it difficult to distinguish between specific intramolecular and non-specific intermolecular effects. In contrast, intermolecular PCSs easily average to zero, as the PCSs depend not only on the distance of the nuclear spin from the paramagnetic centre, but also on the orientation of the metal complex relative to the coordinate frame of the nuclear spin. Orientational averaging of the paramagnetic centre, as encountered for non-specific intermolecular effects, exposes nuclear spins to both positive and negative PCS effects, which average to zero.

\subsubsection{Tolerance towards incomplete tagging yields}

Classical PRE tags, such as nitroxide tags and $\mathrm{Gd}^{3+}$ or $\mathrm{Mn}^{2+}$ tags, do not change the chemical shift of the nuclear spins compared with the diamagnetic reference. As a consequence, failure to achieve $100 \%$ ligation of the tag leads to NMR spectra in which the peaks of paramagnetic and diamagnetic species overlap. This complicates the analysis of quantitative PRE measurements. In contrast, PCSs lead to resolved cross-peaks for the paramagnetic species irrespective of the yield achieved in the ligation reaction.

\subsubsection{Superior distance range}


PCSs decrease with increasing distance $r$ from the paramagnetic centre with a $r^{-3}$ dependence (see Eq. 1 below). In contrast, PREs decrease with $r^{-6}$. The lesser distance dependence of PCSs is useful in many respects, including structure analyses of large proteins, the possibility of attaching tags at sites remote from the site of interest, and in interpreting data obtained from tags that are linked to the target protein by a flexible tether. If the tether is short, the distance variation between the paramagnetic centre and nuclear spins in the protein can be small compared with the distance itself, but this requires that the paramagnetic effects be observable for remote spins. As a result, excellent correlations between experimental and back-calculated PCSs can be observed even for flexible tags.

A particular advantage associated with the long-range effects of PCSs is for studies of protein-protein complexes in situations, where chemical exchange leads to significant line broadening of the NMR signals from nuclear spins that are located at the protein-protein interface. In this case, PCS effects can be observed for nuclear spins with narrower NMR signals that are located at some distance from the interface itself (e.g. in the nearby interior of the protein) and thus too far to engage in intermolecular NOEs. PCSs induced by the most strongly paramagnetic lanthanide ions can be observed over greater distances than PCSs generated by any d-block transition metal ion.

\subsection{Recent reviews on paramagnetic NMR}

Studying proteins with the help of paramagnetic lanthanide ions is becoming increasingly popular. Notably, such analyses represent only a subset of paramagnetic NMR studies. Some excellent recent reviews on paramagnetic effects in NMR are listed below.

Comprehensive reviews on the physical background of paramagnetic effects in biomolecular NMR have been published by Bertini and co-workers [4, 15-17] and summarized in later reviews [18-20]. Reviews of applications in structural biology have been published with emphasis on metalloproteins [21-25], carbohydrates [26], solid state NMR [27-29], PREs for characterising transient, low-population states [30], protein-protein complexes [31], techniques for measuring PCSs [32], sparse protein labelling [33], paramagnetic tags [34-37] and paramagnetically induced residual dipolar couplings (RDC) and relaxation enhancements [18]. Additional reviews have focused on the use of PCSs and paramagnetically induced RDCs for structure analysis of flexible proteins [38] and comparisons of protein conformations in solution and in single crystals [39]. A number of reviews have specifically discussed the advantages of lanthanide ions for structure determinations by paramagnetic NMR, emphasising in particular the value of the 
pseudocontact shifts generated by lanthanides [19, 31, 40-42]. Additional benefits in structural biology have been noted to be associated with the luminescent properties of $\mathrm{Tb}^{3+}$ and $\mathrm{Eu}^{3+}$ ions [43]. A comprehensive review of the chemical requirements for lanthanide coordination and the properties of lanthanide complexes was published by Parker and coworkers in 2002 [44].

The present review aims to provide a comprehensive overview of lanthanide and $\mathrm{Co}^{2+}$ binding tags and the paramagnetic effects generated by these tags in the NMR spectra of biological macromolecules, together with a compilation of applications.

\section{Pseudocontact shifts}

\section{1. $\chi$ tensor and $\Delta \chi$ tensor}

The pseudocontact shift (PCS) of a nuclear spin is manifested in NMR spectra as the change in chemical shift due to the presence of a paramagnetic centre (Fig. 2). In contrast to the Fermi contact shift, which is mediated through chemical bonds, the PCS is a throughspace effect. For a point dipole in a rigid molecule, it can be described by a simple equation that reflects the location, orientation and degree of anisotropy of the magnetic susceptibility associated with the paramagnetic centre:

$$
\Delta \delta^{\mathrm{PCS}}=1 /\left(12 \pi r^{3}\right)\left[\Delta \chi_{\mathrm{ax}}\left(3 \cos ^{2} \theta-1\right)+1.5 \Delta \chi_{\mathrm{rh}} \sin ^{2} \theta \quad \cos 2 \phi\right]
$$

where $\Delta \delta^{\mathrm{PCS}}$ is the PCS (a dimension-less number usually measured and reported in ppm), $r$ is the distance of the nuclear spin from the metal ion, $\Delta \chi_{\mathrm{ax}}$ and $\Delta \chi_{\mathrm{rh}}$ are the axial and rhombic components of the magnetic susceptibility anisotropy $(\Delta \chi)$ tensor, and $\theta$ and $\phi$ are the polar angles describing the position of the nuclear spin with respect to the principal axes of the $\Delta \chi$ tensor (Fig. 3). 


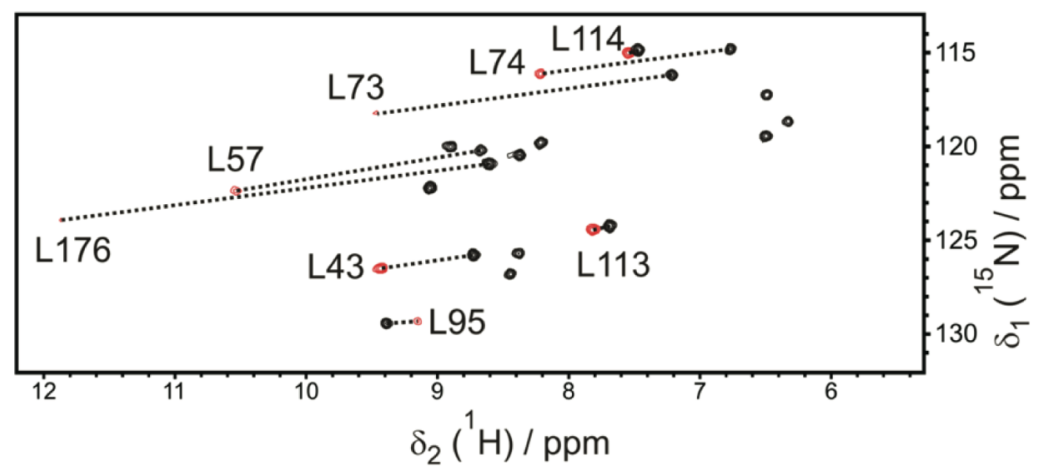

Fig. 2. $\mathrm{PCSs}$ in a $\left[{ }^{15} \mathrm{~N},{ }_{1}^{1} \mathrm{H}\right]-\mathrm{HSQC}$ spectrum. The PCS of a nuclear spin is measured as its chemical shift in the paramagnetic sample, measured in ppm, minus its chemical shift in the diamagnetic sample. The figure shows the superimposition of two spectra, one recorded with paramagnetic $\mathrm{Tb}^{3+}$ (red spectrum), the other with diamagnetic $\mathrm{La}^{3+}$ (black spectrum). The metal ions were bound to the protein $\varepsilon_{186}$ selectively labelled with ${ }^{15} \mathrm{~N}$-leucine. Note that PCSs can be positive (as in the case of, e.g., Leu43) or negative (Leu95). The peaks in the paramagnetic sample are displaced along nearly diagonal lines, because ${ }^{15} \mathrm{~N}$ and ${ }^{1} \mathrm{H}$ nuclei experience very similar PCSs as their separation is small relative to the distance of each from the paramagnetic centre. Cross-peaks of amide protons within about $14 \AA$ of the paramagnetic centre are broadened beyond detection due to PRE. (Reproduced with permission from ref. [42]. Copyright 2007 American Chemical Society.)

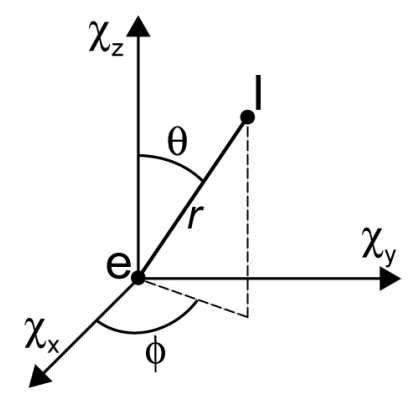

Fig. 3. Geometric parameters defining the PCS of a nuclear spin, I, due to unpaired electrons located in the paramagnetic centre, e.

The magnetic susceptibility tensor, $\chi$, can be regarded as an object with three orthogonal axes, centred about the paramagnetic centre, where the length of each of the axes describes the magnitude of the magnetic moment induced by an external magnetic field, if the axis happens to be aligned with the magnetic field. The $\chi$ tensor is spherical, or isotropic, if all three principal axes have the same length. While the magnitude of the $\chi$ tensor determines 
the paramagnetic relaxation enhancements, PCSs are generated only if the $\chi$ tensor is anisotropic. The $\Delta \chi_{\text {ax }}$ and $\Delta \chi_{\text {rh }}$ components are defined by

$$
\Delta \chi_{\mathrm{ax}}=\chi_{\mathrm{z}}-\left(\chi_{\mathrm{x}}+\chi_{\mathrm{y}}\right) / 2 \text { and } \Delta \chi_{\mathrm{rh}}=\chi_{\mathrm{x}}-\chi_{\mathrm{y}}
$$

where $\chi_{x}, \chi_{y}$ and $\chi_{z}$ denote the susceptibility values along the three principal tensor axes. The $\chi$ and $\Delta \chi$ tensors share the same principal axes and the $\Delta \chi$ tensor vanishes for $\chi_{\mathrm{x}}=\chi_{\mathrm{y}}=\chi_{\mathrm{z}}$.

In an alternative representation, the $\Delta \chi$ tensor can be depicted by isosurfaces that trace the coordinates of a given PCS value as calculated by Eq. 1. It is helpful to distinguish isosurfaces of positive and negative PCSs by different colours (Fig. 4).

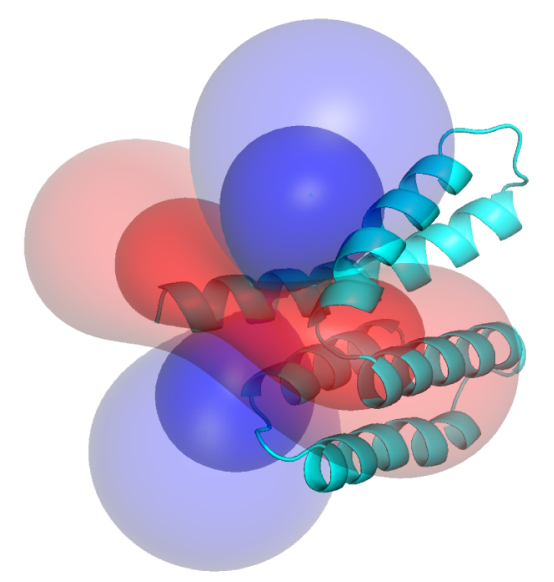

Fig. 4. PCS isosurfaces plotted on the structure of a protein (ERp29 C-terminal domain). The backbone of the protein is drawn in a ribbon representation (cyan). The isosurfaces correspond to PCSs of \pm 0.7 and $\pm 0.2 \mathrm{ppm}$ generated by a $\mathrm{Tb}^{3+}$ ion complex attached to the protein. Positive and negative PCSs are indicated by blue and red isosurfaces, respectively.

\subsection{Unique $\Delta \chi$ tensor representation}

Comparison between different $\Delta \chi$ tensors requires conventions. The unique tensor representation (UTR) established by Schmitz et al. labels the $\chi$ tensor axes such that $\left|\chi_{z}\right|>\left|\chi_{y}\right|$ $>\left|\chi_{\mathrm{x}}\right|$ in analogy to alignment tensor conventions [45]. With this convention, $\Delta \chi_{\mathrm{ax}}$ and $\Delta \chi_{\mathrm{rh}}$ always have the same sign and $\Delta \chi_{\mathrm{rh}}$ is at most $2 / 3$ of $\Delta \chi_{\mathrm{ax}}$. The orientation of the tensor relative to the protein is usually reported in terms of Euler angles $\alpha, \beta$ and $\gamma$ (Fig. 5). The UTR adheres to the "ZYZ" convention for the rotation axes, i.e. the first rotation is by the angle $\alpha$ about the $\mathrm{z}$ axis of the protein frame, the second rotation is by the angle $\beta$ about the new $y^{\prime}$ axis and the final rotation by the angle $\gamma$ is about the new $z^{\prime \prime}$ axis (Fig. 4). By reporting all three Euler angles in the interval $[0, \pi]$ and setting $\gamma=0$ if $\beta=0$, the UTR conventions 
generate a single unambiguous way of reporting a $\Delta \chi$ tensor [46]. It must be kept in mind, however, that these conventions also imply that an increase of $\Delta \chi_{\mathrm{rh}}$ beyond $2 / 3 \Delta \chi_{\mathrm{ax}}$ automatically causes a change in axis definitions and a change in sign of $\Delta \chi_{\text {ax }}$ and $\Delta \chi_{\text {rh. }}$. It is thus quite possible that two lanthanide ions produce PCSs of opposite sign even though their $\Delta \chi_{\text {ax }}$ values are reported to have the same sign.

With the UTR conventions, $\Delta \chi_{\mathrm{ax}}$ is always greater than $\Delta \chi_{\mathrm{rh}}$, so that the magnitudes of two different $\Delta \chi$ tensors can be compared by referring to the axial component $\Delta \chi_{\text {ax }}$ only.
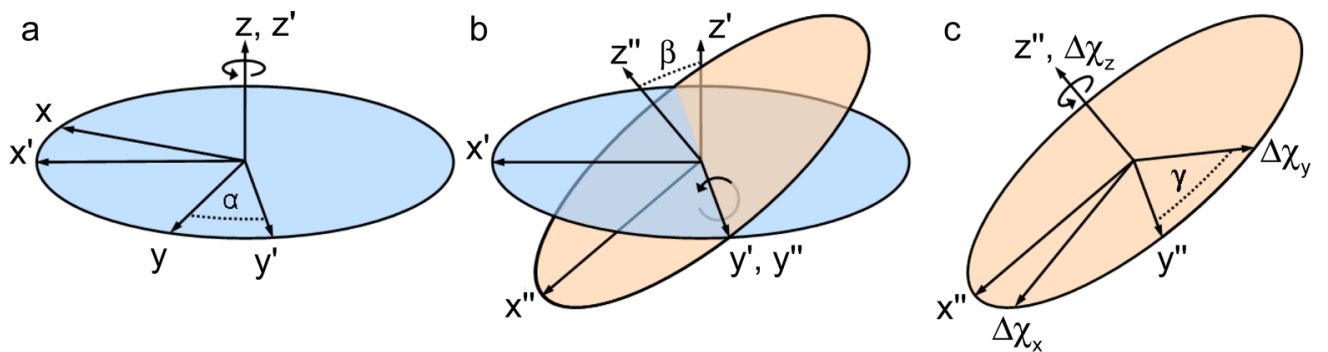

Fig. 5. Euler angle definitions in the ZYZ convention. Euler rotations by the angles $\alpha, \beta$ and $\gamma$ define the relative orientation of the $\Delta \chi$-tensor frame with respect to the protein frame. (a) A right-handed rotation by the angle $\alpha$ around the $\mathrm{z}$ axis is applied to the protein frame xyz to give the frame $x^{\prime} y^{\prime} z^{\prime}$. (b) A second rotation by the angle $\beta$ around the new axis $z^{\prime}$ is applied to the frame $x^{\prime} y^{\prime} z$ ' to give the frame $x^{\prime \prime} y^{\prime \prime} z "$. (c) A final rotation by the angle $\gamma$ around the $z "$ axis maps the protein frame onto the $\Delta \chi$-tensor frame.

\section{Paramagnetic properties of lanthanides}

Fig. 6 provides an overview of the different paramagnetic properties of the paramagnetic lanthanide ions (excluding promethium, which is radioactive). Gadolinium produces exceptionally large PREs, but its $\chi$ tensor is isotropic and, hence, does not generate PCSs. With seven unpaired electrons in the $\mathrm{Gd}^{3+}$ ion, each of the seven f-orbitals can be populated with one electron, explaining the symmetry of the $\chi$ tensor. All other paramagnetic lanthanide ions produce PCSs.

\section{1. $4 \chi$ tensors associated with lanthanide ions}

The largest $\Delta \chi$ tensors are found for lanthanides heavier than $\mathrm{Gd}^{3+}$. In the case of calbindin $\mathrm{D}_{9 \mathrm{k}}$, for which the $\Delta \chi$ tensors are reported in Fig. $6, \mathrm{~Tb}^{3+}$ produced the largest $\Delta \chi$ tensor and $\mathrm{Tm}^{3+}$ generated a $\Delta \chi$ tensor of intermediate magnitude. This ranking, however, depends very much on the ligand field of the lanthanide. For example, in the case of the 
exonuclease domain $\varepsilon_{186}$, the $\Delta \chi$ tensor associated with $\mathrm{Dy}^{3+}$, measured by the magnitude of the $\Delta \chi \quad$ component, is almost $50 \%$ larger than that of $\mathrm{Tb}^{3+}$ [47]. Conversely, in the case of the CLaNP-5 lanthanide tag, the $\Delta \chi$ tensor associated with $\mathrm{Tm}^{3+}$ is $50 \%$ larger than that of $\mathrm{Dy}^{3+}$ [48]. Unfortunately, it is at present not possible to predict $\Delta \chi$ tensors by quantumtheoretical calculations that take the ligand field into account.

Generally conserved features of the $\Delta \chi$ tensors include the relative signs of the largest PCSs produced. Thus, the largest PCSs produced by $\mathrm{Tb}^{3+}$, $\mathrm{Dy}^{3+}$ and $\mathrm{Ho}^{3+}$ are almost always of opposite sign to those produced by $\mathrm{Er}^{3+}, \mathrm{Tm}^{3+}$ and $\mathrm{Yb}^{3+}$. This is not always reflected in the $\Delta \chi$ tensors reported, which can have different signs for the same metal ion (see section 2.2). In the same ligand environment, the directions of the principal axes of the $\Delta \chi$ tensors tend to be similar for different lanthanides, at least for the $\chi_{z}$ component. This feature greatly facilitates the assignment of paramagnetic NMR spectra, by conserving the relative sign and magnitude of PCSs generated by different $\mathrm{Ln}^{3+}$ ions. It also means that PCS measurements of multiple samples that differ only in the type of $\mathrm{Ln}^{3+}$ ion do not deliver entirely independent structural information.

The ideal lanthanide for PCS measurements is characterised by a small isotropic $\chi$ tensor and a large $\Delta \chi$ tensor, to minimise PREs and maximise PCSs. Fig. 6 shows that the magnitudes of the $\Delta \chi \quad \chi$ tensors correlate significantly but not completely. For example, $\mathrm{Dy}^{3+}, \mathrm{Ho}^{3+}$ and $\mathrm{Er}^{3+}$ tend to generate relatively large PRE effects without producing very large PCSs, making them less attractive than, for example, $\mathrm{Tb}^{3+}, \mathrm{Tm}^{3+}$ or $\mathrm{Yb}^{3+}$.

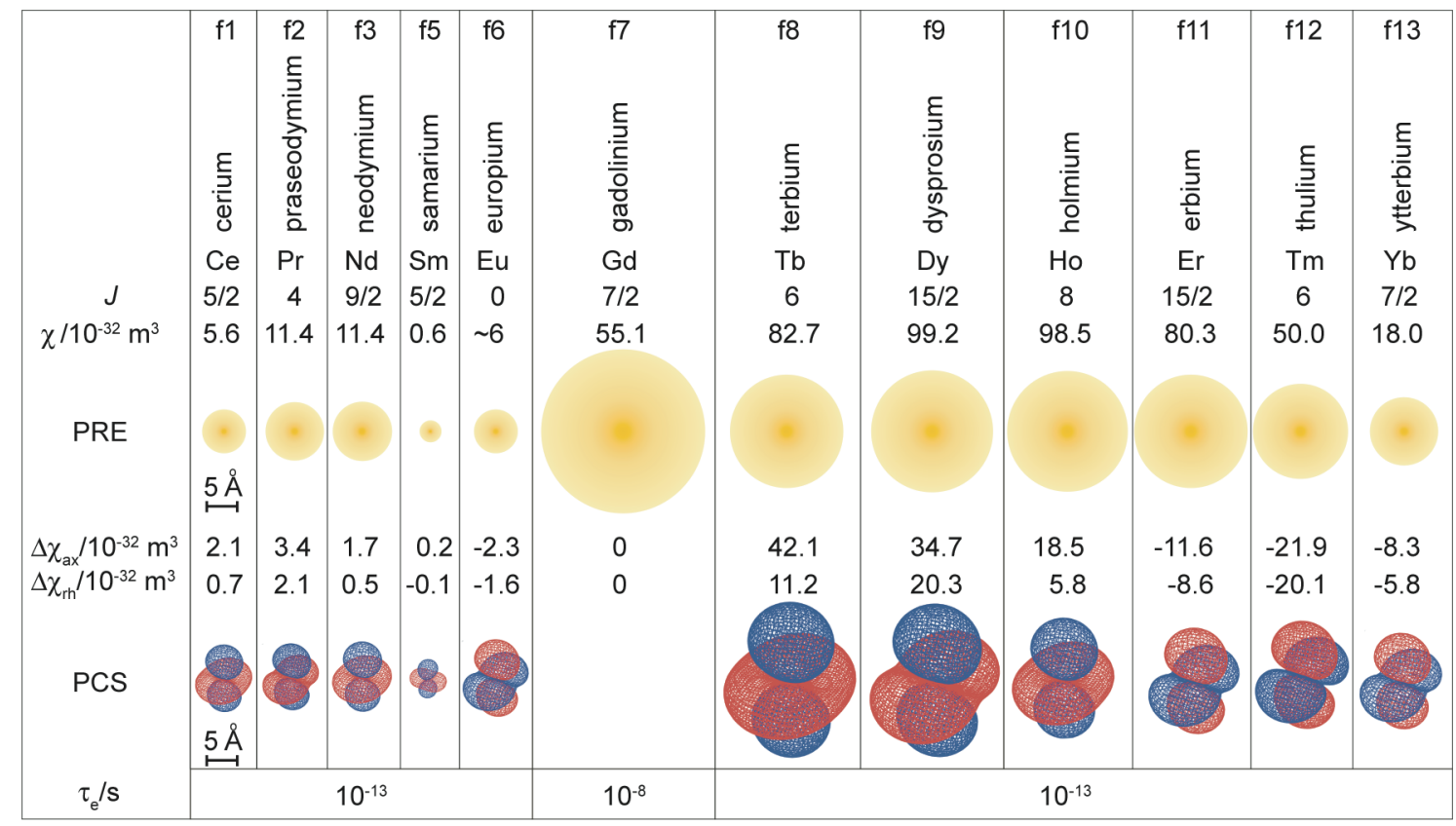


Fig. 6. Properties of paramagnetic lanthanide ions. Promethium was omitted from the figure because it is radioactive. The radii of the yellow spheres indicate the distance from the metal ion where PRE would broaden the ${ }^{1} \mathrm{H}$ NMR signals of a protein with a rotational correlation time of $15 \mathrm{~ns}$ by $80 \mathrm{~Hz}$ on an $800 \mathrm{MHz}$ NMR spectrometer. PCS isosurfaces of $\pm 5 \mathrm{ppm}$ are shown for $\Delta \chi$ tensors reported for calbindin $\mathrm{D}_{9 \mathrm{k}}$ [49]. Electronic relaxation times expected at 18.8 $\mathrm{T}$ are indicated at the bottom [50]. Reproduced with permission from ref. [42]. Copyright 2007 American Chemical Society.

\subsection{Paramagnetic relaxation enhancements by lanthanide ions}

Using lanthanides for PRE measurements is complicated by the extremely short electron relaxation times of all $\mathrm{Ln}^{3+}$ ions apart from $\mathrm{Gd}^{3+}$. Fundamentally, nuclear relaxation is driven by the dipole-dipole interaction between the nuclear spins and unpaired electrons. The precise mechanism by which this dipole-dipole interaction results in nuclear relaxation depends on the electron relaxation time. For slow electron relaxation, as in the case of the $\mathrm{Gd}^{3+}$ ion, $\mathrm{Mn}^{2+}$ ion or a nitroxide radical, the size of the electron magnetic moment does not change as the molecule rotates. This relaxation mechanism has been described mathematically by Solomon in 1955 and in the following is referred to as Solomon relaxation [51]. A different relaxation mechanism, however, applies when the electron relaxation occurs on a time scale much shorter than the rotational correlation time of the molecule, i.e. when the electron magnetic moment continuously changes in size as the molecule rotates. In this situation, the net magnetic moment generated by the unpaired electrons is referred to as Curie spin and the corresponding relaxation mechanism as Curie relaxation [52]. Curie spin relaxation is closely related to relaxation by chemical shift anisotropy (CSA) [53-55].

Lanthanide ions with anisotropic $\chi$ tensors are invariably associated with electron relaxation times in the picosecond range and cause Curie spin relaxation. The anisotropic $\chi$ tensors lead to anisotropic PREs [56] as well as cross-correlation effects between Curie spin relaxation and a range of different relaxation mechanisms [5, 53-55, 57-61]. This makes it difficult to extract accurate distances from PRE measurements with most $\mathrm{Ln}^{3+}$ ions other than $\mathrm{Gd}^{3+}$. Most notably, cross-correlation between CSA and Curie spin relaxation can potentially produce a narrower NMR signal in the presence of a paramagnetic ion than in its absence [53, $62]$.

\section{Determining $\Delta \chi$ tensors}




\subsection{Principles}

$\Delta \chi$ tensors depend on the ligand field of the metal complex and must be determined experimentally for each new metal complex. There are eight parameters to be determined, comprising the $\Delta \chi_{\text {ax }}$ and $\Delta \chi_{\text {rh }}$ values that define the $\chi$ tensor anisotropy, the $\mathrm{x}, \mathrm{y}, \mathrm{z}$ coordinates of the paramagnetic centre, and three rotation angles (e.g. Euler angles) to define the orientation of the $\Delta \chi$ tensor relative to the ligand coordinates. In principle, therefore, measurement of eight pseudocontact shifts of nuclear spins in the complex would be sufficient to determine the $\Delta \chi$-tensor parameters, provided that the three-dimensional (3D) structure of the complex is known. In practice, the NMR resonances of nuclear spins in close proximity to a paramagnetic centre tend to be broadened beyond detection, due to PREs. Moreover, nuclear spins located within a few chemical bonds of the metal ion are subject to Fermi contact shifts, which arise from through-bond interactions with the unpaired electrons, analogous to scalar couplings between nuclear spins [4, 16]. Disentangling PCSs from contact shifts is difficult [63-65]. The problem is particularly stark for lanthanide ions because density functional theory (DFT) calculations, which have been remarkably successful at predicting contact shifts for transition metal ions [1], fail for lanthanide ions due to their large number of electrons. Therefore, determining the $\Delta \chi$ tensors associated with lanthanide ions is most readily achieved by measuring PCSs for a large set of nuclear spins that are located at a sufficiently long distance from the metal ion to avoid contact shifts and PREs, followed by a best fit to available 3D structure coordinates of the protein [49].

\subsection{Quality factors}

Fitting routines for $\Delta \chi$ tensors commonly minimize the sum of the squared deviations between experimental PCSs, PCS exp $_{\text {, and back-calculated PCSs, PCS }}$ calc [46]. As a measure of the quality of the fit, it is customary to report the quality factor

$$
Q=\operatorname{Sqrt}\left[\Sigma\left(\mathrm{PCS}_{\exp }-\mathrm{PCS}_{\text {calc }}\right)^{2} / \Sigma\left(\mathrm{PCS}_{\mathrm{exp}}\right)^{2}\right]
$$

where a smaller number corresponds to a better fit. An alternative definition of the quality factor is also in use $[66,67]$

$$
Q=\operatorname{Sqrt}\left[\Sigma\left(\mathrm{PCS}_{\exp }-\mathrm{PCS}_{\text {calc }}\right)^{2} / \Sigma\left(\left|\mathrm{PCS}_{\exp }\right|+\left|\mathrm{PCS}_{\text {calc }}\right|\right)^{2}\right]
$$

that avoids large contributions from small $\mathrm{PCS}_{\text {calc }}$ values. The definition of Eq. 4 results in smaller $Q$ factors.

\subsection{Effective $\Delta \chi$ tensors arising from flexible metal tags}


When metal tags are used to endow proteins with a paramagnetic centre, almost all tags currently available are attached via somewhat flexible tethers. As a result, the $\Delta \chi$ tensor derived from PCSs measured for the protein represents an 'effective' $\Delta \chi$ tensor that fits the data but is affected by averaging over the different tag orientations and metal positions that arise from the flexibility of the tether. In this situation, the true $\Delta \chi$ tensor of the tag itself remains unknown. For practical applications, the true tensor may actually be of limited interest, as the metal tag is usually deployed to obtain structural restraints in the protein rather than the tag. If a metal complex undergoes rotational motion with respect to the protein without changing the location of the metal ion, the average $\Delta \chi$ tensor is perfectly described by an effective $\Delta \chi$ tensor, which obeys Eq. 1 and can be used to predict the PCSs of all other nuclei in the structure.

As the PCSs depend on the distances of the nuclear spins from the metal ion, a fundamental problem arises when flexibility of the tag allows the metal ion to change its position relative to the protein. In principle, this situation could be simulated by fitting the observed PCSs with multiple $\Delta \chi$ tensors, but fitting so many parameters would require PCSs for a large number of nuclei and still leave doubts about the accuracy of PCS predictions for any other sites in the protein. In practice, it is common to fit the data with a single effective $\Delta \chi$ tensor regardless of the motions of the metal ion. The error associated with this approach has been assessed in a comprehensive theoretical analysis, which determined the accuracy of PCS predictions in regions of the protein that had not been used to fit the effective $\Delta \chi$ tensor [68]. The analysis showed that a surprisingly large amplitude of translational metal ion movement can be accommodated by the effective $\Delta \chi$-tensor approach, in particular, if nuclear spins closer than $10 \AA$ to the metal ion and PCSs greater than $\pm 3 \mathrm{ppm}$ are excluded from the $\Delta \chi$-tensor fit. Obviously, the accuracy with which the PCSs of a group of nuclear spins can be predicted by an effective $\Delta \chi$ tensor deteriorates with their distance from the set of nuclear spins used to fit the $\Delta \chi$ tensor [69]. The predictive value of an effective $\Delta \chi$ tensor can be enhanced by determining the tensor through fitting to a subset of nuclear spins only, which are selected by their proximity to the region of interest $[68,69]$.

\subsection{PCS measurements and assignment of paramagnetic NMR spectra}

In the case of paramagnetic centres with an isotropic $\chi$ tensor $\left(\mathrm{Gd}^{3+}, \mathrm{Mn}^{2+}\right.$, nitroxides), the chemical shifts are unaffected by the paramagnetism. In contrast, the chemical shift changes associated with PCSs require new assignments of the NMR signals in the 
paramagnetic state. While the assignment strategies available for diamagnetic proteins can also be used for the resonance assignments of paramagnetic proteins, PREs present obstacles by broadening many resonances beyond detection. To circumvent these complications, approaches have been developed to transfer resonance assignments from the diamagnetic to the paramagnetic spectra.

\subsubsection{Resonance assignment using $\Delta \chi$-tensor fits}

Transfer of resonance assignments from diamagnetic to paramagnetic spectra is most easily achieved by HSQC spectra, as the ${ }^{1} \mathrm{H}$ spin and the directly bonded heteronucleus experience very similar PCSs owing to their close proximity compared with the distance from the paramagnetic centre. Consequently, the HSQC cross-peaks of paramagnetic and diamagnetic samples are displaced along almost parallel lines with a slope close to one. In the example of amide cross-peaks in a $\left[{ }^{15} \mathrm{~N},{ }^{1} \mathrm{H}\right]-\mathrm{HSQC}$ spectrum, the assignment is facilitated by recording three spectra, two of paramagnetic samples with different lanthanide ions and one of a diamagnetic reference. Superimposition of the three spectra shows three cross-peaks for each amide, which lie on the same line [32]. Using $\mathrm{Tb}^{3+}$ and $\mathrm{Tm}^{3+}$ as the paramagnetic lanthanides, the PCSs tend to be of opposite sign and their magnitude larger for $\mathrm{Tb}^{3+}$ than $\mathrm{Tm}^{3+}$. The resulting peak pattern greatly helps assignment of the paramagnetic cross-peaks. Nonetheless, spectral overlap always leads to ambiguities. To resolve these, an initial $\Delta \chi$ tensor can be fitted using the unambiguous PCSs measured from resolved cross-peaks, which allows prediction of unassigned PCSs and thus the assignment of further paramagnetic peaks. Rounds of $\Delta \chi$-tensor refinements and PCS prediction have been automated, e.g., in the program Echidna [47].

\subsubsection{Resonance assignment by chemical exchange of metal ions}

An alternative assignment strategy is available, if the metal ion exchanges in and out of its binding site. In the fast exchange limit, cross-peaks shift continuously, from the diamagnetic peak position towards the paramagnetic peak position, with increasing concentration of paramagnetic metal ion. If the exchange is slow on the chemical shift time scale but still within the time scale of $T_{1}$ relaxation, a sample prepared with a mixture of paramagnetic and diamagnetic metal ions can generate exchange cross-peaks that link the paramagnetic and diamagnetic auto-peaks. Exchange lifetimes on a suitable time scale have been observed for a protein containing a natural metal binding site [32, 70-72] and for 
ubiquitin tagged with an iminodiacetic acid (IDA) tag to form a lanthanide coordination site together with a carboxyl side chain of the protein [73]. In a different protein, however, the strategy of combining the IDA tag with a protein carboxyl group failed to produce metal exchange rates that were sufficiently fast to generate sizeable exchange cross-peaks [74]. In view of the difficulty of designing metal binding sites with suitable metal exchange rates, other design criteria are usually considered more important, such as location and magnitude of the $\Delta \chi$ tensor as well as thermodynamic stability of the metal complex.

\subsubsection{Using PCSs to assign diamagnetic NMR spectra}

Selective isotope labelling of the target protein presents a straightforward way of spectral simplification that facilitates the assignment of paramagnetic peaks by reducing spectral overlap. With well-resolved cross-peaks in the paramagnetic and diamagnetic HSQC spectra, it becomes possible to measure complete sets of PCSs without having to rely on $\Delta \chi-$ tensor fits and PCS predictions. Combined with the 3D structure of the protein and knowledge of the metal position, the PCSs can then be used to assign not only the paramagnetic but also the diamagnetic cross-peaks, especially if additional paramagnetic information is included, such as PREs, paramagnetically induced RDCs and cross-correlated relaxation effects [75]. In a related approach, it has been shown that, using the $\Delta \chi$ tensor determined from a wellresolved ${ }^{15} \mathrm{~N}-\mathrm{HSQC}$ spectrum to predict PCSs, most side-chain methyl groups of a $21 \mathrm{kDa}$ protein in a $30 \mathrm{kDa}$ complex could be assigned both in the diamagnetic and paramagnetic state by comparison with experimentally measured PCSs [72].

The next step would be to try to assign side-chain methyl resonances without prior knowledge of the $\Delta \chi$ tensor, which would be attractive for high-molecular weight systems where side-chain methyl resonances are much easier to detect than backbone amides. A potential problem associated with this strategy is presented by intrinsic side-chain mobility, which would compromise the quality of the $\Delta \chi$ tensors that can be obtained. The problem associated with side-chain mobility has been highlighted by the difficulty of fitting accurate $\Delta \chi$ tensors to side-chain amides of the $19 \mathrm{kDa}$ protein PpiB using PCSs generated with $\mathrm{Tb}^{3+}$, $\mathrm{Tm}^{3+}$ and $\mathrm{Yb}^{3+}[76]$.

\subsubsection{Additional aids for the assignment of paramagnetic NMR spectra}

Owing to their smaller gyromagnetic ratio, the NMR resonances of heteronuclear spins are less affected by PREs than ${ }^{1} \mathrm{H}$ NMR signals. Many protonless experiments have 
been designed to observe nuclear spins located close to the paramagnetic centre [77]. These experiments are most valuable for probing the electronic properties of the paramagnetic centre and they generate a more complete set of PCSs. Notably, however, resonances with very large PCSs are more difficult to assign and, as $\Delta \chi$-tensor fits minimize the mean square deviation between observed and back-calculated PCSs, very large PCSs have a disproportionate weight in the fit, which tends to degrade the quality of back-calculated PCSs at greater distances from the metal ion. Moreover, close to the paramagnetic centre, the point-dipole approximation underlying Eq. 1 loses its validity even for lanthanide ions [2].

The Curie law implies that magnetic susceptibility increases with the inverse temperature $T^{-1}$, leading to larger PCSs at lower temperature. Plots of chemical shifts versus $T^{-1}$ can thus be used to determine the sign of a PCS and to discriminate between large and small PCSs, but it must be taken into account that the chemical shifts of the diamagnetic reference are also sensitive to temperature $[12,63,78]$.

At very high magnetic fields, the PCSs decrease because of saturation of magnetic moments. The effect is proportional to the square of the magnetic field $B_{0}$ but still very small (of the order of 1\%) at currently available NMR field strengths [79]. It is easily obscured by preferential molecular orientation in the magnetic field, which equally depends on $B_{0}^{2}$ [80] and, for nuclear spins displaying chemical shift anisotropies, generates residual anisotropic chemical shifts (RACS). RACS effects are distance-independent and in paramagnetic molecules can be greater than $0.1 \mathrm{ppm}$ on a $800 \mathrm{MHz}$ NMR spectrometer [81]. As the molecular alignment with the magnetic field originates from the anisotropy of the magnetic susceptibility of the metal complex, a flexibly attached metal tag confers less alignment on the protein it is attached to, downscaling the RACS effects and any other effects resulting from molecular alignment.

\subsection{Paramagnetically induced residual dipolar couplings}

Metal ions with non-vanishing $\Delta \chi$ tensors confer a weak molecular alignment on the molecules they are bound to. The alignment is with respect to the external magnetic field $B_{0}$ and is manifested in the NMR spectra by residual dipolar couplings (RDCs) (in addition to the RACS effects mentioned in Section 4.4.4). The principal axes of the alignment tensor governing the paramagnetically-induced RDCs coincide with the principal axes of the $\Delta \chi$ tensor governing the PCSs. RDCs contain distance-independent information about the orientation of spin pairs with respect to the molecular alignment tensor. For a rigid molecule 
containing a paramagnetic centre with non-vanishing $\Delta \chi$ tensor, the $\mathrm{RDC} D_{\mathrm{AK}}($ in $\mathrm{Hz})$ between the spins $\mathrm{A}$ and $\mathrm{K}$ is given by [15]

$$
D_{\mathrm{AK}}=-\left(h B_{0}^{2} \gamma_{\mathrm{A}} \gamma_{\mathrm{K}}\right) /\left(240 r_{\mathrm{AK}}{ }^{3} k_{\mathrm{B}} T \pi^{3}\right)\left[\Delta \chi_{\mathrm{ax}}\left(3 \cos ^{2} \Theta-1\right)+1.5 \Delta \chi_{\mathrm{rh}} \sin ^{2} \Theta \cos 2 \Phi\right],
$$

where $\gamma_{\mathrm{A}}$ and $\gamma_{\mathrm{K}}$ denote the magnetogyric ratios of the nuclear spins $\mathrm{A}$ and $\mathrm{K}$, respectively, $r_{\mathrm{AK}}$ is the internuclear distance, $h$ is Planck's constant, $k_{\mathrm{B}}$ the Boltzmann constant, $T$ the temperature, and the angles $\Theta$ and $\Phi$ define the orientation of the vector that connects the coupled nuclei $\mathrm{A}$ and $\mathrm{K}$ with respect to the molecular alignment tensor (Fig. 7).

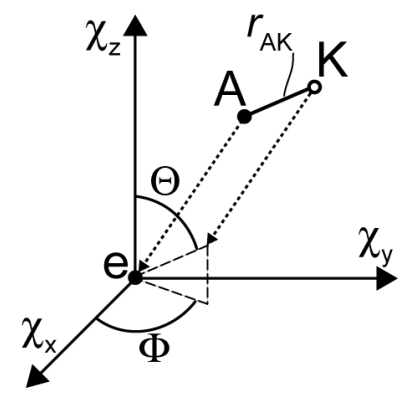

Fig. 7. Geometric parameters defining the RDC between the nuclear spins $\mathrm{A}$ and $\mathrm{K}$, which arises from molecular alignment in the magnetic field owing to an anisotropic $\chi$ tensor with the principal axes $\chi_{\mathrm{x}}, \chi_{\mathrm{y}}$ and $\chi_{\mathrm{z}}$.

\subsection{Using RDCs to assess tag mobility}

For a rigid molecule with a rigidly bound metal tag, the $\Delta \chi$ tensor can be derived from the alignment tensor determined from RDCs using

$$
\Delta \chi_{\mathrm{ax}, \mathrm{rh}}=\left(15 \mu_{0} k T / B_{0}^{2}\right) A_{\mathrm{ax}, \mathrm{rh}}
$$

where $A_{\text {ax }}$ and $A_{\text {rh }}$ are the axial and rhombic components of the alignment tensor, $B_{0}$ is the magnetic field strength, $\mu_{0}$ the magnetic permeability of vacuum, $k$ the Boltzmann constant, and $T$ the temperature [15]. In contrast to a $\Delta \chi$-tensor fit, which is affected by any tag motions that relocate the metal ion relative to the protein, the quality of an alignment tensor fit using RDCs is not compromised by tag mobility, as RDCs do not depend on the distance of the nuclear spins from the paramagnetic centre (eq. 3). Therefore, discrepancies between $\Delta \chi$ tensors derived from PCSs and RDCs can indicate tag mobility. Model calculations indicate that RDCs decrease with increasing amplitude of tag motions, while the effective $\Delta \chi$ tensor 
determined from PCSs tends to increase. The magnitude of both effects, however, is sensitive to the orientation of the original $\Delta \chi$ tensor with respect to the protein, and $\Delta \chi_{\text {ax }}$ values determined from PCSs and RDCs can be the same within $20 \%$ even for tag motions in a cone with an opening angle of $120^{\circ}$ [68]. Therefore, the discrepancy between the tensors derived from RDCs and PCSs is not a good measure of the amplitude of tag motions.

\subsection{Software using PCSs}

\subsubsection{Programs for $\Delta \chi$-tensor determination}

Experimental determination of $\Delta \chi$ tensors from PCSs requires the coordinates of the atoms for which the PCSs have been measured. The required fitting routine has been implemented in many different software packages, such as the recent implementation in the MSpin software by MestreLab Research [82]. Only some of the more frequently used programs are explicitly described here. Fantasian [83, 84] comprises early standalone software for fitting $\Delta \chi$ tensors to proteins, which was designed to fit $\Delta \chi$ tensors for several different metals, provided their coordinates relative to the protein are known. The program Numbat [46] was written later to provide a convenient graphical user interface. It can simultaneously fit $\Delta \chi$ tensors from up to 4 different metal ions and determine the metal position, and includes a routine to take RACS corrections into account. pyParaTools [85] is a set of Python routines to fit $\Delta \chi$ tensors. The program can create libraries of tag conformations and identify the conformation that best fits the experimental PCS data. A very recent implementation is offered by the program FANTEN [86], which fits $\Delta \chi$ tensors and alignment tensors from PCSs and RDCs, using multiple metal ions. The program has a graphical interface, can perform a joint analysis of PCSs and RDCs, and allows rigid body docking of two molecules using $\Delta \chi$ and alignment tensors.

\subsubsection{Programs using PCSs for NMR resonance assignments of proteins}

$\Delta \chi$-tensor fits to protein structures often yield excellent correlations between backcalculated and experimental PCSs. This invites the use of PCSs for NMR resonance assignment of proteins of known 3D structure. As the same PCS value may be observed for different nuclei, PCSs alone are not sufficient to obtain specific resonance assignments. These ambiguities can be resolved in combination with additional paramagnetically generated restraints such as RDCs, PREs and cross-correlation effects between Curie-spin and dipoledipole relaxation. This approach is particularly suitable for the assignment of the ${ }^{15} \mathrm{~N}-\mathrm{HSQC}$ 
cross-peaks of a selectively ${ }^{15} \mathrm{~N}$-labelled protein, as demonstrated for the $30 \mathrm{kDa}$ complex between the $\mathrm{N}$-terminal domain of the $\varepsilon$ subunit, $\varepsilon_{186}$, labelled with ${ }^{15} \mathrm{~N}$-leucine, phenylalanine or valine, and the $\theta$ subunit of Escherichia coli DNA polymerase III [75]. Among the paramagnetic restraints, PCSs and RDCs proved to be particularly valuable for resonance assignment. The algorithm, named PLATYPUS, was programmed in Mathematica (Wolfram Research). It assigns the resonances in both the diamagnetic and paramagnetic states, but needs the metal coordinates as input from the beginning. It is attractive that resonance assignments of the well-resolved ${ }^{15} \mathrm{~N}-\mathrm{HSQC}$ spectrum can be obtained in this manner without the need for expensive ${ }^{15} \mathrm{~N} /{ }^{13} \mathrm{C}$-labelled samples or 3D NMR spectra.

In many cases, the 3D structure and resonance assignments of the diamagnetic protein are known, but the resonance assignments in the paramagnetic state are unknown. To assist with resonance assignments in the paramagnetic state of uniformly ${ }^{15} \mathrm{~N}$ or ${ }^{15} \mathrm{~N} /{ }^{13} \mathrm{C}$-labelled proteins, the program Echidna was devised, which uses the 3D structure of the protein together with peak lists of the ${ }^{15} \mathrm{~N}-\mathrm{HSQC}$ or $3 \mathrm{D}$ HNCO spectra in the paramagnetic and diamagnetic state to determine the PCSs and $\Delta \chi$ tensors at the same time. Using spectra of the $\varepsilon_{186} / \theta$ complex, the program was shown to deliver robust $\Delta \chi$-tensor determinations, including refined metal coordinates [47].

Given the 3D structure of a protein and $\Delta \chi$ tensors determined from PCSs of backbone atoms, PCSs of methyl resonances measured in ${ }^{13} \mathrm{C}-\mathrm{HSQC}$ spectra can be used to obtain sequence-specific resonance assignments in the diamagnetic and paramagnetic states, including stereospecific resonance assignments of the methyl groups of valine and leucine residues. The approach was automated in the program Possum [72] and demonstrated with the ${ }^{15} \mathrm{~N} /{ }^{13} \mathrm{C}$-labelled $\varepsilon_{186} / \theta$ complex. The input to the program requires experimental PCSs and it helps to add information about which methyl groups belong to the same amino acid residue, as evidenced by HCCH-TOCSY cross-peaks.

More recently, the program PARAssign [87, 88] was developed to assign backbone amide and side-chain methyl resonances in proteins, both in the diamagnetic and paramagnetic state, using only PCSs measured in 2D NMR spectra. In contrast to previously published software, the program relies on PCSs measured for multiple samples, each with a paramagnetic metal ion attached at a different site, and on approximate estimates of $\Delta \chi$-tensor magnitude, orientation and position, to obtain initial assignments and $\Delta \chi$-tensor fits. It has been shown earlier that good initial estimates can be obtained with double-arm lanthanide tags such as CLaNP-5 [48]. 
All programs for resonance assignment follow an iterative approach, in which the $\Delta \chi-$ tensor parameters are refined as more and more resonance assignments are being made.

\subsubsection{Programs using PCSs for protein structure computation}

Early on, refinement of protein structures by PCSs was achieved by treating PCSs like other structural restraints, e.g. by including an energy term for PCSs in the program XPLOR [89] or by including PCSs in the target function of DIANA [83] or in programs for energy minimization and molecular dynamics $[90,91]$. In this way, PCSs have been incorporated as structural restraints also in the programs CYANA [92] and Xplor-NIH [93, 94], which currently are the most frequently used programs for $3 \mathrm{D}$ structure determinations of proteins from NMR data. A recent implementation of PCSs and self-orientation RDCs in the crystallography package REFMAC5 [95] demonstrated that protein crystal structures can also be refined using these long-range paramagnetic restraints without compromising the quality of the crystal structure, highlighting the similarity of protein structures in solution and single crystals [96].

The attempt to use PCSs for protein structure determination rather than refinement faces the intrinsic problem that $3 \mathrm{D}$ coordinates of at least a subset of atoms in the protein must be available prior to fitting $\Delta \chi$ tensors. The program PCS-Rosetta achieves this by starting from preliminary structural models (decoys) predicted from the amino acid sequence. To assist the structure predictions, it is helpful to begin with a fragment library that is in agreement with the backbone chemical shifts of the protein, as implemented in the CS-Rosetta [97] structure prediction algorithm. Already at the fragment assembly step, PCS-Rosetta fits $\Delta \chi$-tensors to every decoy structure and the quality of the fit is used to select the best models before further structure refinement [98]. This approach was extended in the program GPSRosetta, which uses PCSs from different protein samples prepared with paramagnetic metal ions at different sites. The program was shown to deliver the accurate $3 \mathrm{D}$ fold of the $\mathrm{C}$ terminal domain of ERp29, using incomplete sets of PCSs of backbone amide protons measured in straightforward ${ }^{15} \mathrm{~N}-\mathrm{HSQC}$ spectra of samples with lanthanide tags at four different sites [99]. In combination with an additional iterative resampling algorithm, the program produces accurate 3D structures even for proteins with over 200 residues, which is difficult to achieve with the original Rosetta algorithm [100]. While the algorithm is generally applicable, it hinges on the availability of suitable metal-ion tagged protein samples.

\section{Lanthanide tags for the generation of pseudocontact shifts}


This section discusses tags that can coordinate lanthanide ions, with a particular emphasis on tags that have been found to generate non-vanishing $\Delta \chi$ tensors in target proteins. Lanthanide tags designed to generate PCSs can also be used to generate PREs, which may sometimes present more informative structural restraints than the PCSs. All lanthanide tags can be used with $\mathrm{Gd}^{3+}$ to generate PREs.

\subsection{Lanthanide binding proteins}

Lanthanides play no obvious role in biology and no lanthanide-specific structural motifs are known in proteins. In vitro, however, $\mathrm{Ln}^{3+}$ ions can sometimes replace naturally occurring metal ions such as $\mathrm{Ca}^{2+}, \mathrm{Mg}^{2+}$ or $\mathrm{Mn}^{2+}$. In this way, a natural metal binding site can be used to label the protein with a paramagnetic $\mathrm{Ln}^{3+}$ ion. Substitutions for a lanthanide have been particularly successful in the case of $\mathrm{Ca}^{2+}$-binding metalloproteins containing an EFhand motif as, among all the biological metal ions, $\mathrm{Ca}^{2+}$ is most similar to $\mathrm{Ln}^{3+}$ ions in its ionic radius and complexation chemistry [101, 102]. Natural EF-hand motifs are usually paired to comprise two calcium binding sites, but the greater charge of $\mathrm{Ln}^{3+}$ versus $\mathrm{Ca}^{2+}$ ions hinders the simultaneous incorporation of two $\mathrm{Ln}^{3+}$ ions.

Early studies reported paramagnetic shifts and $\Delta \chi$ tensors for $\mathrm{Yb}^{3+}$ bound to parvalbumin, which naturally binds two $\mathrm{Ca}^{2+}$ ions $[103,104]$. The selectivity of the four $\mathrm{Ca}^{2+}$ binding sites of calmodulin for $\mathrm{Ln}^{3+}$ ions has been studied extensively, showing that lanthanides preferentially bind to the N-terminal domain while a single mutation, N60D, generates a binding site that is selective for lanthanides even in the presence of excess calcium ions [105-107]. Calbindin $\mathrm{D}_{9 \mathrm{k}}$ is another protein that binds two $\mathrm{Ca}^{2+}$ ions in two EFhands, one of which can selectively be replaced by a single lanthanide ion. Calbindin $D_{9 k}$ containing one $\mathrm{Ca}^{2+}$ and one $\mathrm{Ln}^{3+}$ ion has been used extensively by Bertini and co-workers to determine the $\Delta \chi$ tensors of paramagnetic lanthanides [49, 60, 108].

Similar strategies have been successfully applied to metalloproteins with different metal cofactors. For example, the activity of the exonuclease domain $\varepsilon$ of $E$. coli DNA polymerase III (PolIII) depends on two solvent-exposed $\mathrm{Mg}^{2+}$ or $\mathrm{Mn}^{2+}$ ions in the active site, which can be replaced by a single $\mathrm{Ln}^{3+}$ ion. PCSs measured with a truncated $21 \mathrm{kDa}$ form of $\varepsilon, \varepsilon_{186}$, have been used to determine the structure of its $30 \mathrm{kDa}$ complex with another PolIII subdomain, $\theta[75,109]$. Similarly, the two $\mathrm{Mg}^{2+}$ ions in the structurally related E. coli DNA polymerase I and bacteriophage T4 DNA polymerase can be replaced by a single $\mathrm{Eu}^{3+}$ ion $[110,111]$ but, at least in the latter case, the $\mathrm{Mg}^{2+}-\mathrm{Ln}^{3+}$ substitution compromises the 
enzymatic activity [111]. Substitutions of natural metal ions by lanthanides are therefore limited to special cases and may affect the biological activity and structure of the host protein.

\subsection{Lanthanide binding peptides}

Based on the capacity of EF-hands to bind $\mathrm{Ln}^{3+}$ ions, lanthanide-binding peptides (LBP) have been developed for tagging target proteins. In the first application of this approach, N-terminal fusion to a membrane-bound peptide was shown to generate paramagnetically induced $\mathrm{RDCs}$ with $\mathrm{Yb}^{3+}$ and $\mathrm{Dy}^{3+}$ [112]. Subsequently, an extensive peptide screen yielded 17-residue LBPs with greatly improved $\mathrm{Ln}^{3+}$ binding affinity [113, 114], which maintained the EF-hand fold [115]. N-terminal fusion to ubiquitin generated significant paramagnetically induced RDCs with different $\mathrm{Ln}^{3+}$ ions [116]. Concatenation of two LBPs into a 32-residue double-lanthanide-binding tag and $\mathrm{N}$-terminal fusion to ubiquitin produced even larger RDCs and PREs [117], but fitting the PCSs with a $\Delta \chi$ tensor for each metal would be impractical.

If the 3D structure of the target protein is known, a LBP can also be engineered into a loop of the protein structure [118-121]. This approach achieves a well-defined positioning of the lanthanide with respect to the protein but is limited to loop structures of suitable geometry. An LBP was successfully inserted into three different loops of interleukin-1 $\beta$ (IL1 $\beta$ ) [121] and into a loop of a small immunoglobulin $G$ binding protein $[118,119]$. Nanomolar lanthanide binding affinities were achieved. Using IL1 $\beta$, it was also shown that LBPs can be engineered into two loops simultaneously, allowing $\mathrm{Gd}^{3+}-\mathrm{Gd}^{3+}$ distance measurement by pulse electron paramagnetic resonance (EPR) spectroscopy but making it harder to fit $\Delta \chi$ tensors $[120]$.

In contrast to tags attached by chemical reactions, peptide fusions always deliver complete ligation yields, but they do have the intrinsic drawback that fusions limit the tagging sites to the $\mathrm{N}$ - or C-terminus or to specific loop regions of the target protein. In addition, isotope labelling labels the LBP together with the target protein, leading to increased complexity of the protein NMR spectrum, at least in the diamagnetic reference state.

To gain more freedom in positioning the LBP on the protein, LBPs have also been linked to a single cysteine residue via a disulfide bond $[122,123]$. Preparation of both protein and LBP with a cysteine residue poses the challenge of selectively forming specific disulfide bonds between protein and LBP rather than between two protein or LBP molecules. The practical solution to this problem, which is applicable to any tag with a free thiol group, is to 
activate the cysteine residue of the protein first with DTNB to form an activated disulfide bond. The activation reaction can be monitored by the release of yellow thionitrobenzoate and excess DTNB can readily be separated from the activated protein by centrifugal ultrafiltration. Addition of tag once again releases yellow thionitrobenzoate [122]. Notably, a large excess of LBP can lead to disulfide exchange reactions and decreased yields by favouring disulfide bonds between LBPs. As LBPs are strongly acidic, however, the protein-LBP ligation product can readily be isolated by ion exchange chromatography [36].

LBPs can be furnished with a single cysteine residue at different sites, so that the orientation of the $\Delta \chi$ tensor relative to the protein is easily altered by the choice of LBP. To improve the solubility of the LBP, a tryptophan residue present in the original design [113, 114] can be substituted by alanine [122]. 16-residue LBPs were found to be sufficient to produce significant PCSs in the N-terminal DNA-binding domain of the E. coli arginine repressor $(\operatorname{ArgN})$ after attachment to the single cysteine residue present in $\operatorname{ArgN}[122,123]$. This approach offers the general advantage of chemically ligated tags, in that isotope-labelled proteins display the same number of NMR signals with and without tag, at the expense of possibly incomplete ligation yields.

In a hybrid approach, an LBP can also be fused to the N-terminus of a protein and a second linkage established via a disulfide bond. The two-point anchoring of the LBP decreases the tag mobility, thereby reducing the averaging of anisotropic paramagnetic effects and leading to larger PCSs in the protein [124]. The approach was demonstrated with a cysteine mutant of the B1 immunoglobulin-binding domain of protein $G$ (GB1), where the cysteine residue was positioned at a site suitable for formation of a disulfide bond with an Nterminal cysteine residue in the LBP. Formation of the disulfide bond was achieved with DTNB. A drawback of the approach is that the structure of the protein must be known in advance and that the length of the peptide segment connecting the LBP to the protein must be optimized to avoid conformational exchange phenomena and distortion of the protein structure [124-126].

The difficulty of finding the optimal linkage to the target protein is a general challenge associated with LBPs, irrespective of whether they are attached by $\mathrm{N}$ - or C-terminal fusion or by disulfide bonds. Attachment to a highly solvent-exposed cysteine residue can easily lead to a tag that is too mobile to observe useful PCSs, whereas a linkage that is too short can prevent the LBP from folding [36]. To address this challenge, much effort has been devoted over the past decade to developing non-peptidic tags with broader applicability. 


\subsection{Synthetic tags}

Some biological molecules can be made by total chemical synthesis, in which case a metal-binding chelating group can be part of the synthetic design from the beginning. In the special case of carbohydrates, relatively harsh reaction conditions can be used to achieve chemical modification with a metal-binding chelate. For example, the reducing end of an oligosaccharide can be selectively aminated and reacted in an organic solvent with a carboxyl-group containing tag with formation of an amide bond, and protection groups on the tag can be removed with strong acid afterwards [26]. Similarly, DNA molecules can be synthesized to contain reactive groups, such as thiol or amino groups, or the tag can already be part of the protected nucleotide used in the DNA synthesis. Various approaches have been reviewed previously $[26,36]$.

Attaching synthetic tags to proteins is much more challenging, as proteins contain a more diverse range of functional groups and they are prone to irreversible denaturation or degradation in organic solvents, at non-neutral $\mathrm{pH}$ or at elevated temperature. In native proteins, thiol groups stand out for their nucleophilicity and sensitivity towards oxidation reactions, allowing selective alkylation as well as tag ligation via disulfide bonds. More recently, methods for genetic encoding of unnatural amino acids have opened the door to new bioorthogonal chemistries for site-specific reactions with proteins under mild conditions. In some cases, tags can also be directed to specific sites by non-covalent bonding. Challenges beyond selectivity include broad applicability, stability, rigidity and stereochemical purity.

\subsubsection{Chemical synthesis of ligands with metal tags}

Low-molecular weight disaccharides, such as chitobiose or lactose, have been studied with PCSs generated by synthetically attached lanthanide tags that included a mono- or biphenylic linker between the carbohydrate and an EDTA-based lanthanide-binding moiety [26, 127-129]. More recently, a lanthanide-binding chelating group has been linked to a branched nonasaccharide to assess its conformation and flexibility by PCSs [130]. Based on these results, compound 1 (Fig. 8) was synthesized, which combines a disaccharide (lactose) with a lanthanide-binding moiety and can be used as a sensor to detect carbohydrate-protein interactions [82]. Large PCSs were observed with $\mathrm{Dy}^{3+}$ for most carbohydrate protons, indicating a well-balanced linker length that prevented overly strong PREs without compromising PCSs. Following binding to the lactose-binding protein galectin-3, PCSs were observed in the protein as far as $42 \AA$ from the lanthanide ion but the $Q$-factors were fairly large, suggesting movements of the metal relative to the protein. Similarly, the fucose analog 
2 was synthesized via click chemistry and used to probe interactions with a coat protein of human norovirus [131]. Finally, the low molecular weight anaesthetic sevoflurane was chemically modified with a lanthanide-binding DOTA moiety to yield $\mathbf{3}$ to probe binding to two separately expressed domains of calmodulin [132]. Due to weak binding (millimolar), only small PCSs were observed in the protein, limiting the accuracy with which the binding sites could be pinpointed on the protein surface.

These examples illustrate the intrinsic difficulty associated with metal tags attached to ligands, as the tag must not interfere with the protein-ligand interaction, yet needs to be sufficiently immobilised to generate PCSs in the protein that can be interpreted with a single $\Delta \chi$ tensor.
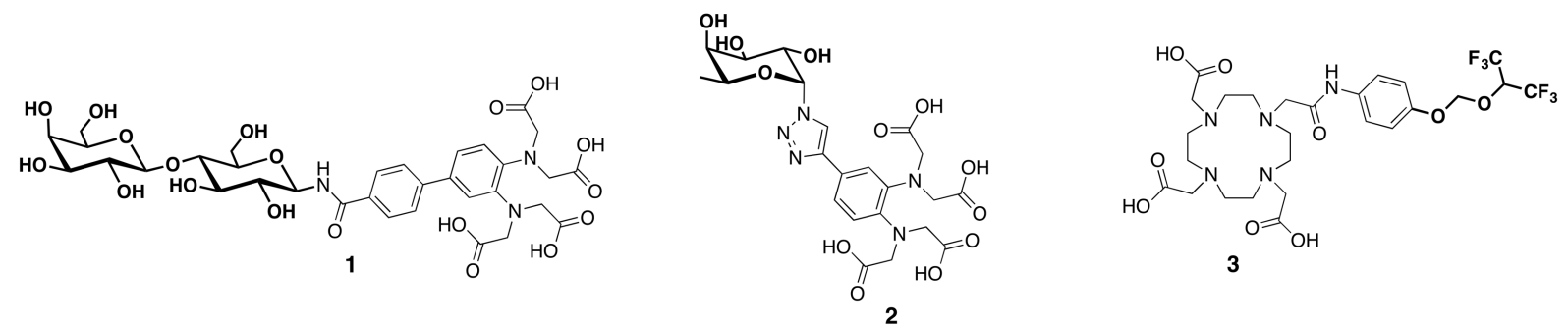

Fig. 8. Ligand molecules fused with lanthanide-binding moieties. The parts comprising the original ligand molecules are highlighted in bold.

\subsubsection{Covalent protein tags}

Various chemical strategies have been reported to achieve selective covalent bond formation between proteins and paramagnetic tags. All examples reported to date either attach the tags to cysteine residues via disulfide or thioether formation, or 4-azidophenylalanine residues are targeted in a "click chemistry" approach (Fig. 9). 


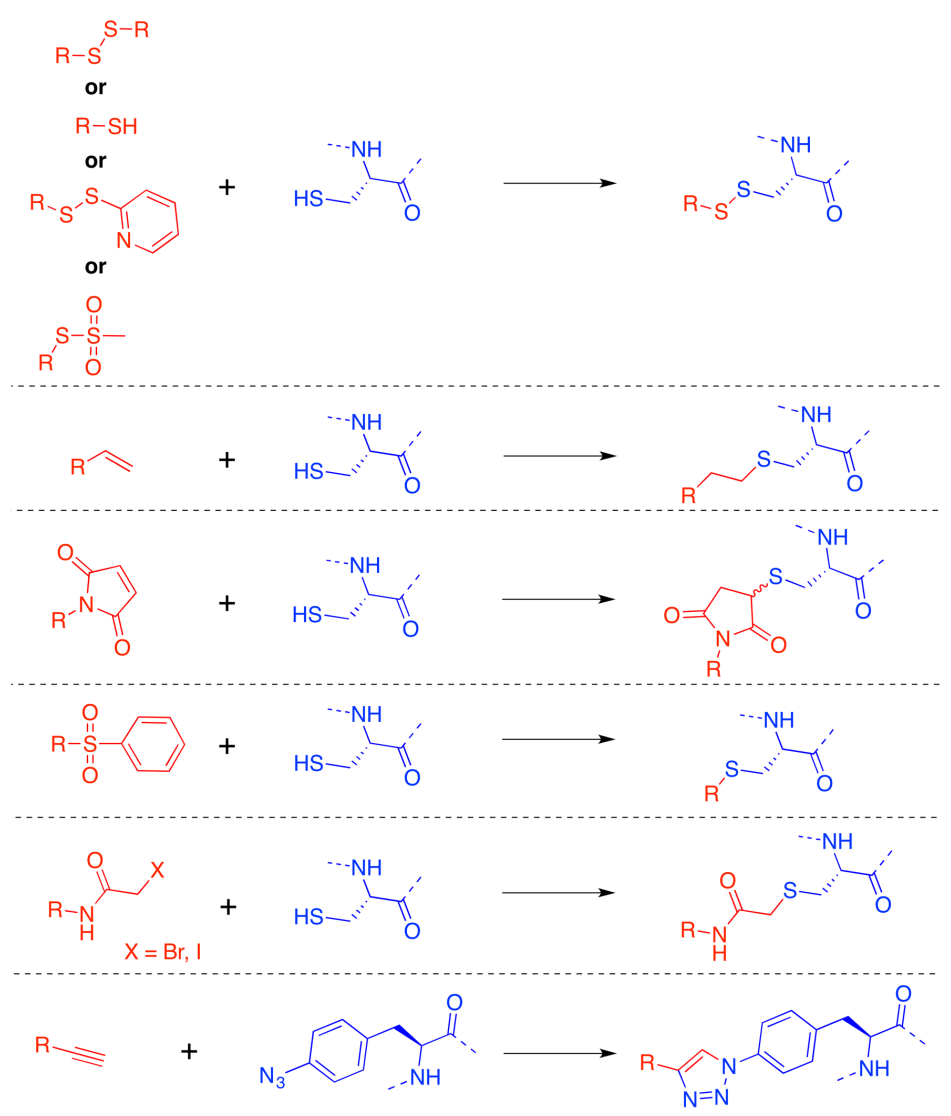

Fig. 9. General reaction schemes for covalently attached tags discussed in this review. The tag and its reactive moiety are shown in red. The targeted amino acid residue is shown in blue. The metal chelating part of the tags is not further specified and is referred to as R. Note that the exact chemical nature of the complete tag (not just its reactive moiety) contributes to the reactivity of a tag. For example, electron-withdrawing groups are often required. The figure does not specify any necessary catalysts or reaction conditions (e.g. pH). Particular care has to be taken with the iodoacetamide group, which can also react with the amino group at the Nterminus of a protein and with histidine or lysine side-chains [133].

\subsubsection{EDTA tags and peak doubling due to chiral metal complexation}

Most reported tags are attached to cysteine via a disulfide bond. The first non-peptidic NMR probes for tagging proteins with metal ions were based on EDTA. This universally used hexadentate chelating agent coordinates metal ions, with high stability constants. Modification with a thiol-reactive group, such as in the tags $S$-(2-pyridylthio)cysteaminylEDTA (4) and $S$-methanesulfonylcysteaminyl-EDTA (5), produces EDTA tags that spontaneously form a disulfide bond with a solvent-exposed cysteine residue. While EDTA itself is achiral, EDTA-metal complexes form two enantiomeric forms (Fig. 10). As proteins are always chiral, ligation with a racemic EDTA-metal complex leads to diastereomers and, 
consequently, peak doubling in the NMR spectrum, which becomes particularly apparent with PCSs, as the different isomers are associated with different $\Delta \chi$-tensor orientations relative to the protein $[134,135]$. This makes it difficult to derive $\Delta \chi$ and alignment tensors from PCS and RDC measurements.
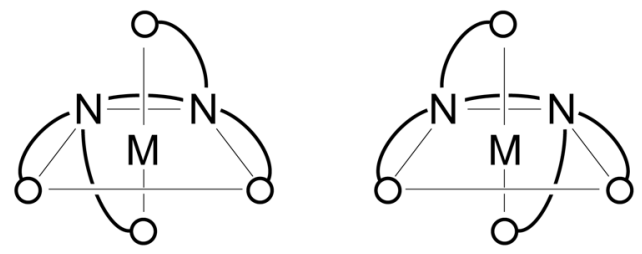

Fig. 10. EDTA forms two enantiomers in complex with a metal ion. Circles symbolise the carboxyl groups of EDTA, curved lines represent the ethylene groups and $\mathrm{M}$ denotes the metal ion.

Tag 4 was used to tag the N-terminal domain of signal transducer and activator of transcription $4\left(\mathrm{STAT}_{\mathrm{NT}}\right)$, where peak doubling observed with $\mathrm{Co}^{2+}$ was interpreted in terms of breaking the symmetry of the homodimer [136]. The tag allowed useful PRE measurements with metal ions with isotropic $\left(\mathrm{Mn}^{2+}\right)$ or nearly isotropic $\left(\mathrm{Cu}^{2+}\right)$ magnetic susceptibility [135-137]. Tag 4 was also linked to a membrane protein for RDC measurements with $\mathrm{Tb}^{3+}, \mathrm{Tm}^{3+}$ and $\mathrm{Yb}^{3+}$, and peak doubling was observed but not commented on [138].

In principle, chiral metal complexation does not cause problems, if the metal complex interconverts sufficiently rapidly between the two chiralities. Notably, however, if fast interconversion between different chiralities is associated with release of the metal ion from the chelating agent, the metal ion can potentially bind also to other sites in the protein. In addition, fast metal dynamics can lead to smaller PCSs by averaging of different $\chi$-tensor anisotropies. In principle, population of a single tag enantiomer may be favoured by interactions with the protein, but this is difficult to engineer.

Fortunately, a single chiral centre in the tag can bias the geometry of the metal chelate to populate almost exclusively a single chiral form. For example, due to rapid inversion of tertiary amino groups, compounds containing a pseudoasymmetric nitrogen, as in the EDTA derivatives $\mathbf{4}$ and $\mathbf{5}$, cannot be isolated in enantiopure form. With a chiral centre elsewhere in the chelating molecule, however, the final metal complex assumes a single preferred chirality. This strategy has been successfully applied to a range of acyclic synthetic tags. 
In the case of EDTA tags, a stereocentre at the attachment point of the linker moiety connecting the metal chelate with the protein (tags $\mathbf{6 a} / \mathbf{b}$ of Fig. 11) proved to produce a single set of RDCs and significant PCSs with $\mathrm{Dy}^{3+}$, following attachment of the tag to a cysteine mutant of trigger factor (TF). Very different $\Delta \chi$-tensor orientations were obtained for epimers TF-6a and TF-6b [134]. 6a and $\mathbf{6 b}$ were also successfully used with a cysteine mutant of ubiquitin [139] and the closely related tags $\mathbf{7 a}$ and $\mathbf{7 b}$ were shown to produce RDCs and PCSs, albeit of modest magnitude, for TF and a cysteine mutant of apo-calmodulin [140]. Importantly, however, the $\mathrm{Tb}^{3+}$ binding affinity of the tag was found to be nine orders of magnitude greater than that of apo-calmodulin, ensuring that the lanthanide bound selectively to the tag and not the protein. Therefore, these tags are suitable for studying metalloproteins.
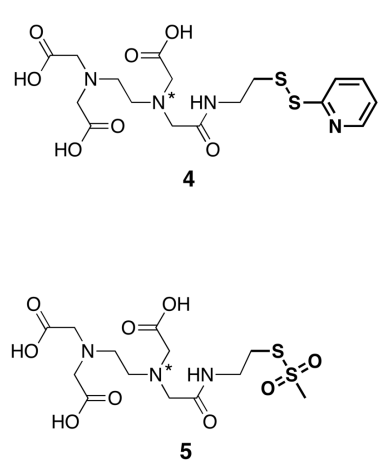
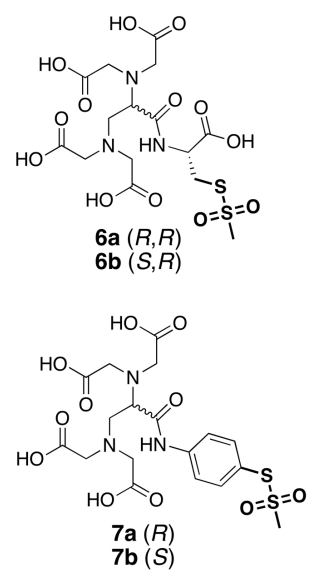
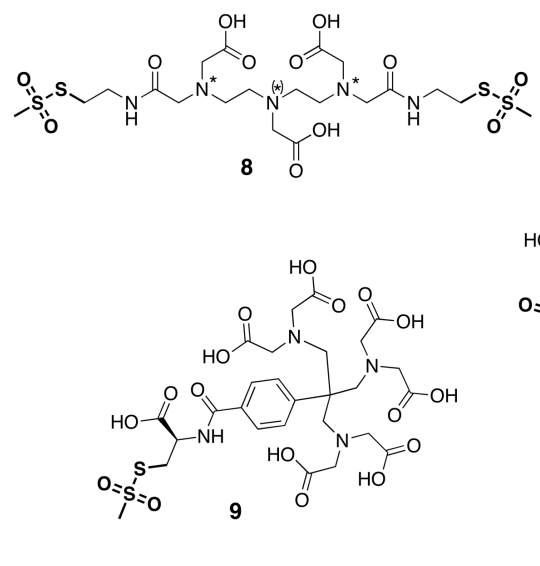

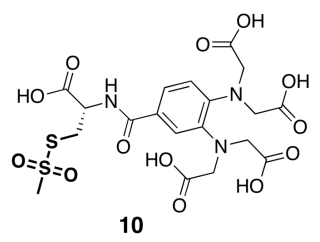

10

Fig. 11. Thiol-reactive acyclic probes. The reactive parts for covalent cysteine tagging are highlighted (bold). Tags $\mathbf{4}, \mathbf{5}, \mathbf{6 a} / \mathbf{b}$, and $\mathbf{7 a} / \mathbf{b}$ are EDTA-based (hexadentate), and probes $\mathbf{8}$ and 9 are derived from DTPA (octadentate) and TAHA (nonadentate), respectively. Asterisks identify pseudoasymmetric nitrogen atoms.

\subsubsection{DTPA tag}

DTPA is an octadentate chelating agent with an even higher binding affinity for $\mathrm{Ln}^{3+}$ ions than EDTA, but the complex between DTPA and a lanthanide produces four pairs of enantiomers as the central symmetric nitrogen also becomes chiral in the complex [44]. Consequently, up to eight different diastereomeric species can be generated by linkage to a protein. Tag 8, referred to as caged lanthanide NMR probe (CLaNP), was designed to address this challenge by tying the tag to two solvent-exposed cysteine residues positioned with about 8-10 ̊ separation. The two-arm attachment rigidifies the tag and raises the possibility that the tag preferentially populates a single stereoisomer by interaction with the protein surface. A crystal structure of this tag tied to pseudoazurine showed that the tag did not affect the protein 
structure [141]. Even with $\mathrm{Yb}^{3+}$, PCSs could be observed at distances up to $40 \AA$ from the metal centre, attesting to the rigidity of the tag. As there were no close contacts between the $\mathrm{Ln}^{3+}$-chelating moiety and the protein, however, up to five different sets of paramagnetic peaks were observed, critically compromising the utility of this tag. Nonetheless, $\Delta \chi$ tensors could be determined from the three most intense sets of PCSs. While for small proteins it may be possible to unravel the NMR effects of three different $\Delta \chi$ tensors, the data analysis becomes prohibitively difficult for larger proteins. In general it is preferable to obtain multiple tensors from different samples, where each paramagnetic or diamagnetic sample produces a single set of NMR peaks.

\subsubsection{TAHA tag}

Following attachment of the nonadentate TAHA-based tag 9 to two different cysteine mutants of ubiquitin with $\mathrm{Tm}^{3+}$ and $\mathrm{Tb}^{3+}$, four sets of PCSs were measured and the corresponding $\Delta \chi$ tensors determined [142]. Each sample yielded a single set of peaks, indicating the absence of diastereomers. The PCSs and RDCs were sizeable and $\Delta \chi$-tensor fits to the known ubiquitin structure produced good (i.e. small) quality factors. The alignment tensors determined from the RDCs were smaller than expected for the size of the $\Delta \chi$ tensors, indicating mobility of the tag relative to the protein, which is a common observation for tags attached to a single cysteine residue. Remarkably, sizeable PCSs were observed also for a 90 $\mathrm{kDa}$ sample of dimeric Lac repressor tagged with the TAHA tag and in complex with DNA and IPTG, and the complex was stable for at least two weeks at elevated temperature [142].

\subsubsection{Cyclen tags}

The cyclic ring structure of tetraethyleneamine alone (commonly referred to as cyclen) does not bind lanthanides with significant affinity. In contrast, the tetraacetic acid derivative DOTA (Fig. 12) binds lanthanides and other metal ions with very high affinities, producing complexes that are also kinetically inert with regard to the release of metal ions under biological conditions. Loading a DOTA-type complex with a lanthanide ion is also slow and often requires high temperatures, so that the lanthanide complexes must be prepared before they can be ligated with a protein. It may seem like a disadvantage that their extraordinary stability prohibits metal exchange after tagging of the protein, but in practice it is a big advantage not having to titrate the tagged protein with lanthanide ions, as the exact protein concentration is often unknown making accurate titrations difficult. The Cys-Ph-TAHA tag 9 
has also been described for use in Ln-preloaded form [142], but other non-cyclen tags are usually loaded with lanthanides after attachment to the target protein.

DOTA tags have long been used in magnetic resonance imaging (MRI) and nuclear medicine [143]. Both the ring structure and the acetic acid pendants, however, can assume two different conformations, resulting in two pairs of enantiomeric species after lanthanide binding [144]. These conformers interconvert with a rate that is slow on the NMR time scale and therefore present distinct sets of PCSs $[145,146]$.

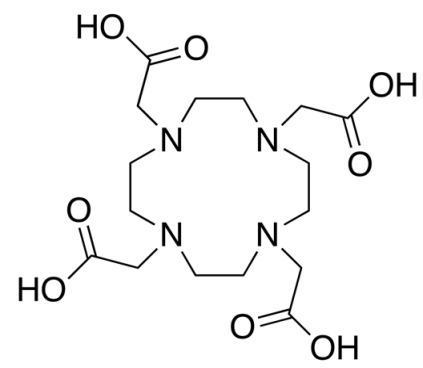

Fig. 12. Chemical structure of DOTA.

\subsection{Double-arm cyclen tags}

Early tests with the double-arm DOTA tag 11 (CLaNP-3) tied to pseudoazurine showed that bidentate anchoring to the protein was not sufficient to select a single tag conformation (Fig. 13) [147]. While the experiments revealed observable PCSs up to $35 \AA$ from $\mathrm{Yb}^{3+}$, peak doubling was pronounced. In addition, ${ }^{15} \mathrm{~N}-\mathrm{HSQC}$ cross-peaks were broadened beyond detection for amides within $18 \AA$ of the metal ion. The latter effect, however, cannot be attributed solely to PREs, as cross-peaks could be observed for amides much closer to the metal for tag 12 attached to the same protein and cysteine mutant, indicating exchange broadening by conformational exchange within the tag as an alternative explanation [141].

Starting from these initial results, a number of DOTA-based probes have been evaluated. Double-arm cyclic DOTA tags such as $\mathbf{1 1}$ are symmetric in their free form, but lose their symmetry upon binding to a protein. The major advantage of double-arm tags arises from their better capacity to immobilize the metal ion relative to the target protein. While tag 11 did not completely suppress the occurrence of peak doubling, the CLaNP-5 tag 12 showed no evidence for the formation of diastereomers [48, 148]. The substitution of two acetate pendants by pyridyl- $N$-oxide moieties effectively selects a single one of the diastereomeric DOTA- $\mathrm{Ln}^{3+}$ species in solution $[149,150]$. 
CLaNP-5 (12) has subsequently become a widely used lanthanide tag, as it avoids peak doubling and has an exceptional capacity to generate large $\Delta \chi$ tensors in proteins with $\mathrm{Yb}^{3+}$ and $\mathrm{Tm}^{3+}$, which are lanthanide ions that do not generate overly large PREs (Fig. 6). The superior metal immobilization and, consequently, larger $\Delta \chi$ and alignment tensors achieved by double-arm attachment of CLaNP-5 has been documented by comparison with the singlearm analogue 13 [148]. CLaNP-5 (12) has also been demonstrated to perform well at different sites of pseudoazurin, laying the foundation for the generation of different $\Delta \chi$ tensors at different sites, which ultimately allows pinpointing the location of a nuclear spin from PCSs alone [48]. Importantly, the $\Delta \chi$-tensor magnitudes measured for $\mathrm{Yb}^{3+}$ were largely conserved at the three different protein locations, revealing this tag to be quite independent from the local environment. With $\mathrm{Tm}^{3+}, \Delta \chi_{\mathrm{ax}}$ tensor magnitudes of over $50 \times 10^{-32} \mathrm{~m}^{3}$ have been observed with excellent correlation between experimental and back-calculated PCSs. In contrast to the $\Delta \chi$ tensors determined for calbindin $\mathrm{D}_{9 \mathrm{k}}$ (Fig. 6), CLaNP-5 generates larger $\Delta \chi$ tensors with $\mathrm{Tm}^{3+}$ than $\mathrm{Dy}^{3+}$. Despite two-arm attachment, the CLaNP-5 tag is still mobile to some extent, as indicated by relaxation dispersion experiments performed with different tagging sites on pseudoazurine and cytochrome $c$ [151]. The degree of tag mobility depends on the tagging site on the protein. This was most clearly shown by paramagnetic relaxation dispersion experiments performed with CLaNP-5 attached to adenylate kinase, where the data revealed a minor and major tag conformation differing predominately by a $23^{\circ}$ rotation around one of the tensor axes, with an exchange rate of $400 \mathrm{~s}^{-1}$ [152]. CLaNP-5- $\mathrm{Ln}^{3+}$ complexes carry a net charge of +3 , which may in some cases affect the protein structure and function by altering its overall charge [40]. It has also been noted that the tagged constructs can be prone to precipitation and the tag can be lost within days or weeks. It is therefore recommended to prepare several double-cysteine mutants and use the most stable ones for further studies [67].

Further development produced CLaNP-7 (14), which contains two phenolic groups instead of $N$-oxides. Nitro groups lower the $\mathrm{p} K_{\mathrm{a}}$ values of the phenol hydroxyls so that deprotonation reduces the net charge of the tag-lanthanide complex to +1 [153]. Tag 14 was attached to pseudoazurine and cytochrome c. Comparison with CLaNP-5 (12) revealed slightly smaller PCSs and $\Delta \chi$-tensor magnitudes as well as different tensor orientations and metal positions. While the tag produced only a single set of PCSs with pseudoazurine, it produced two sets of peaks with cytochrome $c$. The effect of peak doubling was found to be pH-dependent, which could be explained by additional coordination of the $\mathrm{Ln}^{3+}$ ion by the 
imidazole ring of a histidine side chain to replace a water molecule at the ninth coordination site of the lanthanide ion, changing the symmetry of lanthanide coordination.

A more recent variation of CLaNP-5 is presented by CLaNP-9 (15), which contains two bromoacetanilide groups to react with cysteine residues by formation of chemically stable thioether rather than disulfide bonds. Tag 15 was studied with the model proteins pseudoazurine and T4 lysozyme [154]. As expected, the protein-tag complexes were inert towards reducing conditions, but the $\Delta \chi$ tensors were slightly smaller than with CLaNP-5 (12), suggesting greater mobility of the tag with respect to the protein, which may be a consequence of the overall longer tether between metal ion and protein. Unfortunately, the reactivity of tag $\mathbf{1 5}$ makes handling delicate and it is difficult to obtain quantitative ligation yields with target protein.

The latest double-arm tags $\mathbf{1 6 a}$ and $\mathbf{1 6 b}$ result in the same chemical linkers to two cysteine residues as the CLaNP-5 tag, but the other two pendant arms are chiral isopropanol moieties to coordinate the lanthanide ion [155]. Large $\Delta \chi$ tensors were obtained with doublecysteine mutants of ubiquitin and $S$. aureus 6-hydroxymethyl-7,8-dihydropterin pyrophosphokinase (HPPK).
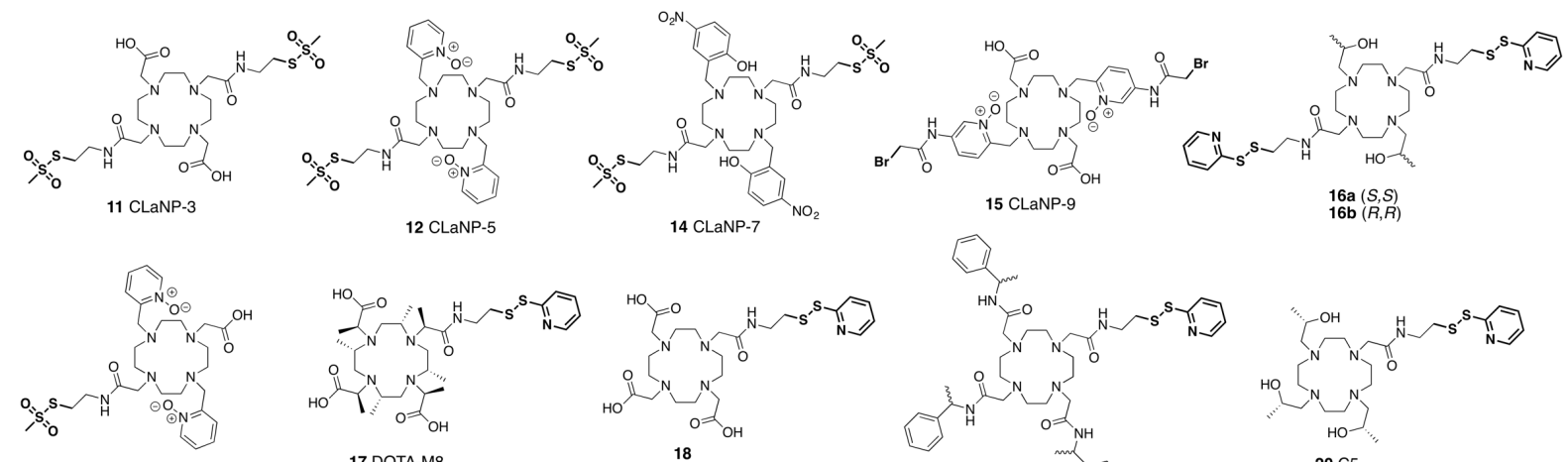

13
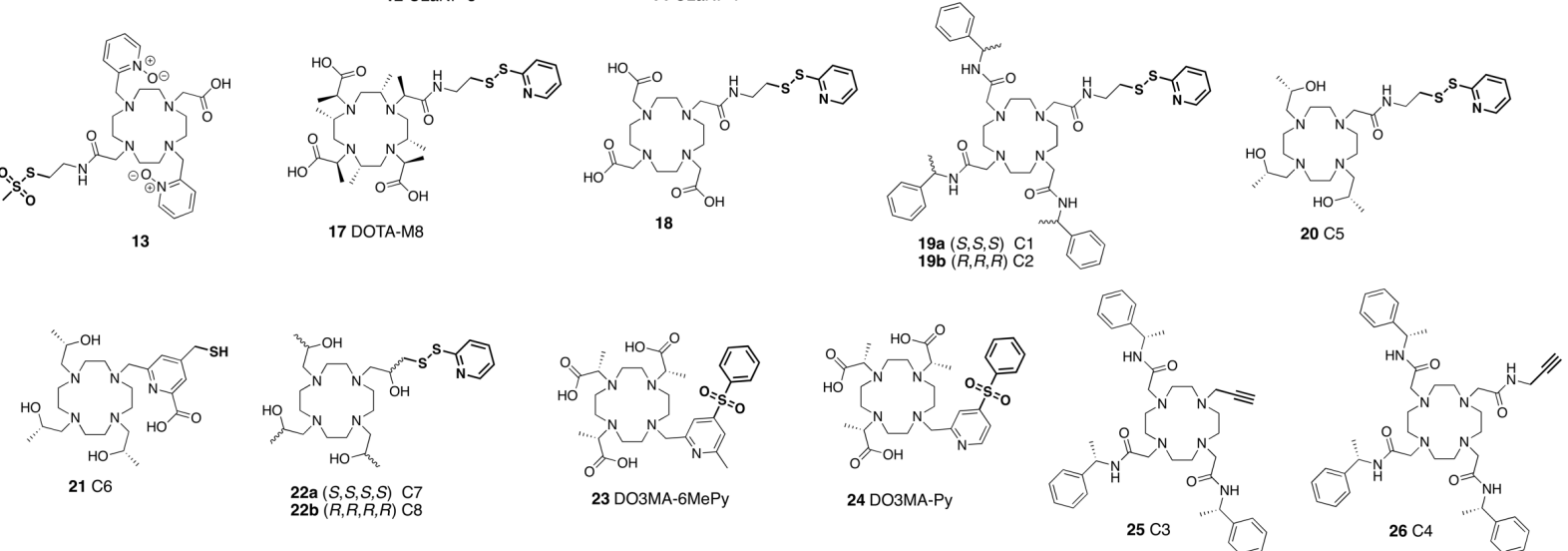

Fig. 13. Thiol-reactive and bioorthogonal cyclen probes and ligation products. The reactive groups for covalent attachment to cysteine (11-24) or for click reactions $(\mathbf{2 5}, \mathbf{2 6})$ are drawn in bold. Activating groups for covalent attachment to cysteine are drawn in bold. Tags 11, 12, 14, 15 and $16 \mathbf{a} / \mathbf{b}$ are double-arm tags.

\subsection{Single-arm cyclen tags binding to cysteine}


Double-arm tags carry two reactive groups for ligation to two reactive groups on the target protein so as to reduce metal mobility; however, such tags also carry the potential to form covalent dimers and multimers. An alternative is presented by single-arm tags, which require only a single reactive group on the protein. As a drawback, single-arm tags are almost always associated with greater metal mobility relative to the protein.

The first single-arm cyclen tag was the DOTA-M8 tag 17. This tag contains eight methyl groups and is enantiomerically pure at all corresponding stereo-centres, to bias the conformational equilibria of the cyclen ring and its pendant arms towards a single conformation. Loaded with $\mathrm{Dy}^{3+}$ and linked to two different sites of ubiquitin, the tag delivered very large PCSs and $\Delta \chi$ tensors, whereas the corresponding tag without methyl groups (tag 18) produced only very small PCSs and displayed broad lines due to conformational exchange [156]. In both samples, the tag also generated a second species (15$20 \%$ at $25{ }^{\circ} \mathrm{C}, 50 \%$ at $50{ }^{\circ} \mathrm{C}$ ), which displayed a $\Delta \chi$ tensor similar in magnitude, but different in orientation and metal position compared to the main species. Initially, the minor species was suggested to arise from cis/trans isomerization of the peptide bond within the linker, but it was later shown to stem from a slow conformational equilibrium between two different lanthanide coordination geometries, referred to as square antiprism (SAP) and twisted square antiprism (TSAP) [157]. The population ratio between the two forms depends on the ionic radius of the lanthanide. At $25{ }^{\circ} \mathrm{C}$ and $\mathrm{pH} 6.5$, the complex with $\mathrm{Pr}^{3+}$ populates the SAP conformation to $98 \%$, whereas the complex with $\mathrm{Yb}^{3+}$ is $95 \%$ in the TSAP conformation and both conformations are approximately equally populated in the complex with $\mathrm{Ho}^{3+}$. In complex with the DOTA-M8 tag, only lanthanide ions with larger ionic radii can coordinate a hydration water molecule, which results in greater temperature sensitivity of the $\Delta \chi$ tensors [158].

In a very recent variation of the DOTA-M8 tag 17 , referred to as M8-CAM-I, the ethylenedithiopyridyl group in tag 17 was replaced by a propyliodoacetamide ($\mathrm{C}_{3} \mathrm{H}_{6} \mathrm{NHCOCH}_{2} \mathrm{I}$ ) group for reaction with cysteine by formation of a thioether bond, which is stable under the reducing conditions of in-cell NMR [159]. Due to the greater length of the tether, smaller $\Delta \chi$ tensors were observed for two different cysteine mutants of ubiquitin than with the original DOTA-M8 tag 17 and the quality factors of the $\Delta \chi$-tensor fits were generally worse. Conformational heterogeneity with SAP and TSAP conformations was avoided by measuring PCS data only for $\mathrm{Dy}^{3+}$. 
Tag 19a (named C1) solves the problem of multiple species [160]. Initially studied with ubiquitin and $\operatorname{ArgN}$, this tag contains amide instead of carboxylate pendants, yet it still binds lanthanide ions with high affinity. Loading the tag with a lanthanide ion requires harsh conditions $\left(80^{\circ} \mathrm{C}\right.$, overnight) and therefore must be completed before the tag can be reacted with a protein. The $\mathrm{C} 1$ tag produces only a single set of paramagnetic peaks and, dependent on the solvent exposure of the tagging site, the $\Delta \chi$ tensors can be large as the bulkiness of the tag limits motions of the lanthanide relative to the protein. The magnitudes of the $\Delta \chi$ tensors follow the usual sequence observed for calbindin $D_{9 k}$ (Fig. 6), with $\Delta \chi_{a x}$ magnitudes decreasing in the sequence $\mathrm{Dy}^{3+}>\mathrm{Tb}^{3+}>\mathrm{Tm}^{3+}>\mathrm{Yb}^{3+}$. The tag contains three chiral centres in its pendant arms.

Tag 19b (C2) is the enantiomer of $\mathrm{C} 1$ of opposite chirality. In combination with $\mathrm{C} 1$, the $\mathrm{C} 2$ tag is useful for producing a different $\Delta \chi$ tensor with the same protein tagging site. Both tags have been used at three different sites of the dengue virus protease NS2B-NS3. Using $\mathrm{C} 1-\mathrm{Tm}^{3+}, \mathrm{C} 1-\mathrm{Tb}^{3+}, \mathrm{C} 2-\mathrm{Tm}^{3+}$ and $\mathrm{C} 2-\mathrm{Tb}^{3+}$ tags, 12 sets of PCSs were obtained to elucidate the conformation of the protein [161]. The bulky, hydrophobic pendant arms shield the net charge of the tags $(+3)$ to some extent, but may generate unintended binding sites for hydrophobic ligand molecules.

The $\mathrm{C} 5$ tag (20) is a variant of the $\mathrm{C} 1$ tag, containing three pendant arms consisting of chiral $(S)$-2-hydroxypropyl groups instead of phenethylacetamide groups [162]. Additional variants of C5 were produced with different tethers to the cysteine sulfur atom, where C6 (21) contains a different, more rigid, picolinic acid-derived linker and C7 (22a) and C8 (22b) employ yet another tether that generates a more symmetric coordination sphere for the lanthanide ion [162]. In addition, C8 was synthesised, which is the enantiomer of C7. Following ligation to the A28C mutant of ubiquitin, PCSs measured with $\mathrm{Dy}^{3+}, \mathrm{Tb}^{3+}, \mathrm{Tm}^{3+}$ or $\mathrm{Yb}^{3+}$ produced the lowest $Q$ factors for the $\mathrm{C} 7$ and $\mathrm{C} 8$ tags. Their $\Delta \chi_{\mathrm{ax}}$ values were larger on average than those obtained with the $\mathrm{C} 1$ and $\mathrm{C} 2$ for the same ubiquitin mutant. In contrast to $\mathrm{C} 1$ and $\mathrm{C} 2$, which reliably generate PCSs of opposite sign with $\mathrm{Tb}^{3+}$ and $\mathrm{Tm}^{3+}$, the relative order and size of PCSs induced by the C5 - C8 tags proved to be variable, suggesting changes in coordination environment with each lanthanide ion. Unexpectedly, some of the largest $\Delta \chi$ tensors were obtained with $\mathrm{Tm}^{3+}$ (also confirmed with a cysteine mutant of GB1) and an unusually large $\Delta \chi$ tensor was obtained with the C7- $\mathrm{Tm}^{3+}$ tag attached to a single-cysteine mutant of HPPK [162]. 
The C9 tag forms a tether to cysteine as short as the C7 and C8 tags but contains phenethylacetamide pendants like the $\mathrm{C} 1-\mathrm{C} 4$ tags. To date, data have been published only for $\mathrm{C} 9$ loaded with $\mathrm{Gd}^{3+}$ for distance measurements by EPR spectroscopy, where it produces particularly narrow distance distributions [163].

Lanthanide complexes of the recently published DO3MA-6MePy (23) and DO3MAPy (24) tags have a net neutral charge and only a single aromatic group [164]. In addition, these tags form thioether bonds with cysteine residues, resulting in chemically stable ligation products and short tethers between protein backbone and lanthanide ion, generating very large $\Delta \chi$ tensors. As a drawback, the ligation reactions are slow even at $\mathrm{pH} 9$, raising the possibility of incomplete ligation yields.

\subsection{Single-arm cyclen tags binding to p-azido-L-phenylalanine}

The chemical inertness of cyclen-lanthanide complexes opens up opportunities to consider chemistries other than disulfide or thioether linkages for site-specific tagging. As most wild-type proteins contain cysteine residues, altering the protein construct to contain only a single reactive thiol group at a selected site can be cumbersome and have unintended consequences for the protein stability and activity. $p$-azido-L-phenylalanine is an unnatural amino acid that can readily be encoded by an amber stop codon [165], and selectively reacted with a tag containing an acetylene group in a cycloaddition reaction that can be performed in the presence of cysteine.

$\mathrm{Cu}^{+}$-catalysed click-reaction with the septadentate tag 25 (C3) has been demonstrated with three different proteins. The resulting tether between lanthanide ion and protein backbone is quite long, but the triazole formed presents a rigid unit that can also coordinate to the metal ion. The alternative octadentate tag $\mathbf{2 6}(\mathrm{C} 4)$ produced greater ligation yields but smaller PCSs, which proved more difficult to fit by a single $\Delta \chi$ tensor, as expected for a more flexible tether [166]. His 6 tags were found to inhibit the ligation reaction, which may be attributed to their copper binding capacity. Subsequent work showed that many proteins are sensitive to precipitation in the presence of copper, even when the copper catalyst is provided as a complex with a multi-dentate ligand such as BTTAA $[167,168]$. 

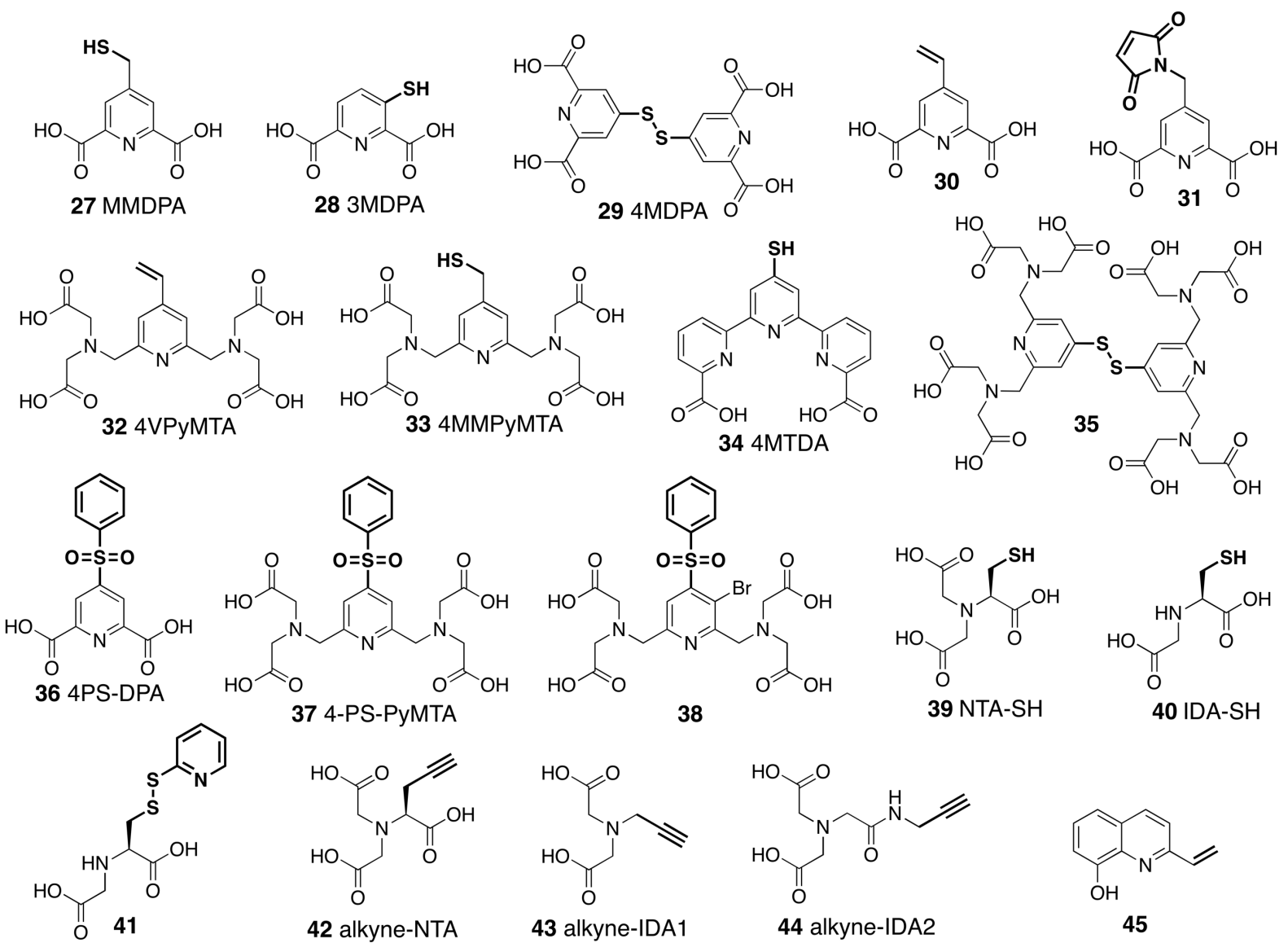

39 NTA-SH

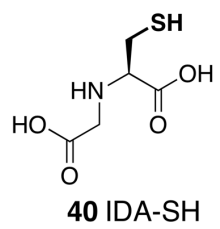

Fig. 14. Probes for covalent (thiol-reactive or bioorthogonal) tagging based on pyridine or iminodiacetic acid (IDA) or nitrilotriacetic acid (NTA) derivatives. Some of the smallest probes are based on dipicolinic acid (DPA). The 8-hydroxyquinoline probe $\mathbf{4 5}$ has only been used as a tag with transition metal ions. The reactive groups for covalent attachment to cysteine $(\mathbf{2 7 - 4 1}, \mathbf{4 5})$ or click reactions $\mathbf{( 4 2 - 4 4 )}$ are drawn in bold.

\subsubsection{Tags based on dipicolinic acid}

Dipicolinic acid (DPA) binds lanthanide ions with nanomolar binding affinities despite being no more than a tridentate ligand (Fig. 15). The complex between a DPA molecule and $\mathrm{Ln}^{3+}$ ion is non-chiral, avoiding the problem of peak doubling associated with chiral metal complexes [169]. In addition, DPA-based tags are much smaller than any EDTA or cyclen tags, minimizing their possible impact on the protein structure. The first DPA tag developed for PCS measurements was 4MMDPA (27). Following attachment to ArgN, a comparison between the $\Delta \chi$ tensors determined from PCSs and alignment tensors determined from RDCs indicated good immobilization of the metal ion, but the $\Delta \chi$ tensors were smaller in magnitude than for, e.g., cyclen tags [170]. Conceivably, filling most lanthanide coordination sites with water molecules does not promote large $\chi$-tensor anisotropies. On the 
other hand, the accessibility of the lanthanide ion allows additional coordination by side chain carboxyl groups from the protein, contributing to immobilization of the metal by an effective two-point attachment as suggested for the ArgN-4MMDPA construct. As the thiol group of tag $\mathbf{2 7}$ is not activated, attachment to the cysteine residue of a protein requires prior activation of the cysteine thiol group with, e.g., DTNB, as in the case of lanthanide binding peptides.

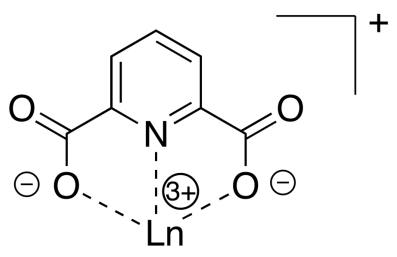

Fig. 15. Chemical structure of the complex between dipicolinic acid (DPA) and a lanthanide ion.

An even smaller tag than 4MMDPA is presented by 3MDPA (28), which is less symmetrical than 4MMDPA but produces a shorter linker with the protein as the reactive thiol group is directly linked to the aromatic ring. 3MDPA is one of the smallest reported lanthanide tags [171]. The tag was studied with ArgN using seven paramagnetic lanthanide ions and $\mathrm{Co}^{2+}$. As expected, the tag produced different $\Delta \chi$-tensor orientations from the 4MMDPA tag. The smaller magnitude of the $\Delta \chi$ tensors seemed to arise from the difficulty of coordinating with a side-chain carboxyl group of ArgN. Dy ${ }^{3+}$ produced the largest PCSs, whereas $\mathrm{Er}^{3+}$ unexpectedly produced smaller PCSs than $\mathrm{Tm}^{3+}$ and $\mathrm{Co}^{2+}$. The 3MDPA tag may depend more critically on additional immobilization, either by steric restraints or by metal coordination with protein side chains, as only very small PCSs could be observed with a cysteine mutant of T4 lysozyme [171].

The disulfide-linked dimer of 4MDPA (29) was synthesized to produce a more symmetrical analogue of the 3MDPA tag. The dimer can be used directly as tagging reagent, as 4-mercapto-DPA is a good leaving group [172]. Lanthanide complexes of ArgN tagged with 29 displayed similar $\Delta \chi$-tensor magnitudes to those with the 3MDPA tag, whereas significantly larger $\Delta \chi$ tensors were observed with the 4MDPA tag attached to the intracellular domain of the rat p75 neurotrophin receptor, which was attributed to additional metal coordination by a side chain carboxyl group of the protein [172]. The effective $\Delta \chi$ tensors generated by the small DPA tags thus seem to be particularly sensitive to additional coordination by protein carboxylates. 
Whereas tags 27-28 are all attached to the protein of interest via disulfide bridges to cysteine residues, the DPA-based tag $\mathbf{3 0}$ can be added to cysteine residues via a thiol-ene reaction to create chemically more inert thioether tethers [173]. The alkene function acts as a Michael acceptor for thiol groups, no further activation of the tag or protein is required, and an excess of tagging reagent can be used, greatly facilitating high ligation yields. The resulting ligation product resembles the product obtained with the 4MMDPA tag 27, except that one of the sulfur atoms is replaced by a methylene group. Following attachment to ArgN, similar $\Delta \chi$ tensors were obtained to those with 4MMDPA. Following attachment to the ubiquitin mutant $\mathrm{T} 22 \mathrm{C}$, the metal ion was found in the proximity of the carboxyl group of Asp21, indicating immobilisation by this additional interaction. The Michael addition reaction produces a stable product, which is inert towards reducing agents, but the reaction rate is much slower than that of tags activated with methylsulfonyl or thiopyridyl groups. Therefore, inaccessible cysteine thiol groups buried inside proteins remain unmodified.

Michael addition reactions using a vinyl group offer an elegant escape from the dilemma that conventional maleimide tags create a new chiral centre upon ligation to thiol groups, resulting in diastereomers with proteins and, hence, peak doubling in NMR spectra. This has been demonstrated experimentally with a DPA tag (31) attached to maleimide [173].

The concept of Michael additions via vinyl groups was subsequently extended to a larger tag with greater lanthanide ion affinity. 4VPyMTA (32) shows even higher binding affinities toward lanthanides than EDTA [174]. The tag was tested with two different cysteine mutants of ubiquitin. In each case, only a single set of cross-peaks was observed in the presence of a paramagnetic lanthanide ion. Despite the greater bulkiness of 4VPyMTA compared with 4MMDPA (27), tag flexibility resulted in relatively small $\Delta \chi$ tensors. Recently, the 4MMPyMTA tag (33) has been reported, which reacts with a cysteine residue by formation of a disulfide linkage [175]. The resulting tether is the same as for the 4VPyMTA tag, except that a $\mathrm{CH}_{2}$ group is replaced by a sulfur atom.

Similarly, the terpyridine-based tag 4MTDA (34) is a bulkier tag, related to DPA but designed to deliver a greater binding affinity. Its ligation to proteins via a disulfide bond follows the conventional approach, where cysteine residues are first activated with DTNB. Titration experiments with DPA confirmed that the lanthanide binding affinity of ubiquitin tagged with 4MTDA was very high. Attached to a cysteine residue in position $i$ of an $\alpha$-helix, the tag allows additional metal coordination by the carboxyl group of a glutamate residue in position $i-4$ of the same helix, resulting in large $\Delta \chi$ tensors and outstanding quality factors 
[176]. Similarly good results were obtained for a non-helical attachment site with a glutamate carboxyl group in the vicinity of the tag. While the observed $\Delta \chi$ tensors were significantly larger than for other DPA tags, the ligation yields were only about 50-70\%.

Another bulky tag with high lanthanide affinity is 35 [177]. It reacts spontaneously with a cysteine residue. Using the $\mathrm{D} 82 \mathrm{C}$ mutant of $S$. aureus sortase A, complete ligation was observed within 8 hours without affecting the more buried cysteine residue at the active site.

A direct bond between a pyridine ring, as in DPA, and a cysteine thiol group presents the shortest conceivable tether. As a good leaving group, the phenylsulfonyl group allows this bond to be formed in aqueous solution, resulting in a thioether. The approach was demonstrated by the 4PS-DPA tag (36), the 4-PS-PyMTA, (37) [178] and 38 [177]. Tag 36 showed that the ligation reaction is selective and complete after about 10 hours at $25^{\circ} \mathrm{C}$. Like other DPA tags, however, additional coordination by a protein carboxylate is required to obtain useful PCSs. Attached to the highly solvent-exposed site of Gly47 of ubiquitin, the 4PS-PyMTA tag 37 produced sizeable PCSs, throughout the protein, that were much larger than for tag $\mathbf{3 2}$ at the same site. This result demonstrates the value of a shorter tether (two methylene groups fewer than in tag 32) at a site where no protein carboxylates are nearby. Tag 38 was ligated to the $\mathrm{D} 82 \mathrm{C}$ mutant of $S$. aureus sortase A at $\mathrm{pH} 7.6$ overnight in about $80 \%$ yield and produced intermediate size $\Delta \chi$ tensors with good quality factors. The ligation rate is faster at $\mathrm{pH}$ 8. Importantly, tag $\mathbf{3 8}$ did not react with the less solvent-exposed cysteine residue in the active site.

Regardless of whether additional complexation by a side-chain carboxyl group of the protein occurs or not, cyclen-based tags tend to deliver larger $\Delta \chi$ tensors than DPA-based tags.

\subsubsection{Tags based on NTA}

Nitrilotriacetic acid (NTA)-based tags were designed in an attempt to minimize the size of the tag without compromising metal binding affinity. As in the case of DPA tags, $\mathrm{Ln}^{3+}$ ions complexed by an NTA tag retain unoccupied coordination sites, allowing immobilization of the metal ion by additional coordination to a protein carboxylate. Following attachment to ArgN and cysteine mutants of ubiquitin, the NTA-SH tag 39 produced single sets of paramagnetic cross-peaks for all four paramagnetic lanthanides analysed. As the metal binding affinity of NTA is lower than that of EDTA, EDTA can be used to remove the bound lanthanide to allow substitution by another lanthanide [179]. In the case of ArgN, the PCSs measured were comparable in magnitude to those observed with 4MMDPA (27), although the 
lanthanide ion appeared to be at a distance of $4.7 \AA$ from the nearest carboxyl group of the protein, which would be too far for direct complexation. In contrast, ubiquitin produced smaller PCSs despite the presence of two protein carboxyl groups in proximity to the metal ion. Compared with the lanthanide-DPA complexes, the lanthanide-NTA complex seems to engage less readily in complexation by additional carboxyl groups of the protein, possibly due to its smaller net charge ( 0 for the NTA- $\mathrm{Ln}^{3+}$ complex, +1 for DPA- $\mathrm{Ln}^{3+}$ ). Most interestingly, a two-point anchoring approach has been realized with the NTA-SH tag $\mathbf{3 9}$ by attaching a tag to each of two cysteine residues located at positions $i$ and $i+4$ of the $\alpha$-helix in ubiquitin. This generated large PCSs, excellent correlations between experimental and back-calculated PCSs, and also an alignment tensor in agreement with the $\Delta \chi$ tensor [179].

\subsubsection{Tags based on IDA}

To reduce the size of the lanthanide tag even further, the iminodiacetic acid (IDA)based tags 40/41 contain only two carboxyl groups. The complex with $\mathrm{Ln}^{3+}$ ions carries a positive net charge $(+1)$, promoting additional coordination by a carboxyl group of the protein. This was demonstrated with the A28C mutant of ubiquitin, where the IDA-SH tag 40 was found to position the lanthanide ion close to the carboxyl group of Asp32 located in the same $\alpha$-helix [73]. The magnitudes of the $\Delta \chi$ tensors observed for six different paramagnetic lanthanide ions followed the sequence observed for calbindin $\mathrm{D}_{9 \mathrm{k}}$ (Fig. 6), with a very large $\Delta \chi$ tensor for $\mathrm{Dy}^{3+}$, excellent correlations between experimental and back-calculated PCSs, and very good agreement between the $\Delta \chi$ tensor and alignment tensor determined with $\mathrm{Tb}^{3+}$, indicating outstanding rigidity for such a small probe. Most interestingly, addition of an excess of a 1:1 mixture of diamagnetic $\mathrm{La}^{3+}$ and paramagnetic $\mathrm{Dy}^{3+}$ allowed the observation of exchange cross-peaks between the auto-peaks of the diamagnetic and paramagnetic protein, facilitating the assignment of the paramagnetic NMR spectrum [73], using an NMR experiment applied previously to measure PCSs in $\varepsilon_{186}$ [70]. Unfortunately, exchange crosspeaks have proven difficult to observe in other proteins (see Section 4.4.2).

\subsubsection{Prediction of assisted complexation by protein carboxylates}

The performance of the NTA- and IDA-based tags 39 and 40 relies strongly on opportunities for additional coordination by neighbouring side-chain carboxyl groups in the protein. A systematic study conducted to assess the predictability of such additional coordination positioned either the NTA-SH tag 39 or the IDA-SH tag $\mathbf{4 0}$ at position $i$ of an $\alpha-$ 
helix in the C-terminal domain of ERp29, and either an aspartate or glutamate residue at position $i+4$ or $\mathrm{i}-4$ in the same helix. For NTA-SH tag, the largest $\Delta \chi$ tensors were obtained with a glutamate residue in position $i-4$. For the IDA-SH tag, only an aspartate residue in position $i+4$ produced significant $\Delta \chi$ tensors [74]. The $\Delta \chi$ tensors obtained with the IDA-SH tag were somewhat smaller than those reported previously for ubiquitin [73] and exchange cross-peaks between diamagnetic and paramagnetic samples were difficult to observe, suggesting that the performance of the tag is sensitive to small conformational differences between $\alpha$-helices in different proteins.

It is interesting to note that all NTA- and IDA-based tags are expected to coordinate lanthanide ions in a chiral fashion, but peak doubling has never been observed in NMR spectra of proteins ligated with these tags, probably because of rapid exchange between the diastereomeric species or because the chiral centre next to the attachment site of the tether biases the population of the diastereomers.

\subsubsection{NTA and IDA tags for ligation by click chemistry}

Recently, "clickable" versions of NTA- and IDA-based tags have been reported, comprising the alkyne-NTA tag $\mathbf{4 2}$, the alkyne-IDA1 tag $\mathbf{4 3}$ and the alkyne-IDA2 tag $\mathbf{4 4 ,}$ which can be attached to $p$-azido-L-phenylalanine [167]. In this work, the azido group was found to be prone to inactivation by the reducing conditions of the E. coli cytosol, ultimately limiting the ligation yields to between 50 and $80 \%$. The alkyne-NTA tag was tested for two sites of ubiquitin and three sites of GB1, and the alkyne-IDA1 and alkyne-IDA2 tags were tested for the same sites of GB1. Titrating the constructs with $\mathrm{Tb}^{3+}$ or $\mathrm{Tm}^{3+}$, sizeable $\Delta \chi-$ tensor magnitudes were observed irrespective of the presence of additional protein carboxylgroups to assist the coordination of the lanthanide ion, which was attributed to the limited conformational freedom of the tether between the protein backbone and the lanthanide ion. Notably, however, all these tags result in rotatable, barely constrained bonds next to the metal complex, which allows the metal ions to move to some extent, making the tensor magnitudes and quality factors hard to predict. It was concluded that fewer bonds (tag $\mathbf{4 3}$ versus tag $\mathbf{4 4}$ ) make better tags, even if the additional degrees of rotational freedom of a longer tether may put a protein carboxyl group within reach. In the absence of additional coordination by protein carboxyl groups, NTA tags may be preferable over IDA tags in view of their greater affinity for lanthanides [167]. 


\subsubsection{Commercial availability}

Owing to a largely experimental character, some of the tags discussed above have been reported in single articles only. Nonetheless, a number of tags are commercially available at the time of this review. These include the EDTA tags 4 and 5 (Fig. 11), the CLaNP-5 tag 12, the DOTA-M8 tag 17 (Fig. 13), the DPA tags MMDPA 27, 3MDPA 28, 4vinyl-DPA 30, the alkyne-IDA tags 43 and 44 and the 2-vinyl-8-hydroxyquinoline tag 45 (Fig. 14).

\subsubsection{Non-covalently binding tags}

Site-specific attachment of tags to a target protein does not necessarily depend on the formation of covalent bonds, but can also be achieved by non-covalent association. In principle, this approach does not require any chemical modification of the protein, allowing recovery of the protein after the experiment. If the tag exchanges rapidly between the proteinbound and free state, the paramagnetic cross-peaks can be assigned in a straightforward titration experiment to observe gradual spectral changes from the free protein towards the tagged protein. Unfortunately, binding specificity and rapid chemical exchange between bound and free tag tend to be mutually exclusive requirements, and it is a challenge to obtain a defined orientation of the $\Delta \chi$ tensor relative to the protein for a rapidly exchanging tag. Only a few examples of PCSs generated in this way have been reported to date.

\subsubsection{Non-covalently binding synthetic tags}

Many proteins that are not metallo-proteins, such as ubiquitin, contain weak binding sites for the aquo ions of paramagnetic lanthanides, which can generate small PCSs. Soluble lanthanide complexes, however, can bind more specifically. In an early example, DTPA complexes of different lanthanides were shown to change chemical shifts in the protein FKBP, which were proposed to assist resonance assignments by resolving accidental chemical shift degeneracies [180]. No $\Delta \chi$ tensors were determined.

$\left[\operatorname{Ln}(\mathrm{DPA})_{3}\right]^{3-}$ complexes carry an even greater charge than Ln-DTPA complexes and were successfully used for PCS, RDC and PRE measurements [181-184]. The dipicolinic acid molecules bind the lanthanide ion in a nonadentate complex with propeller-like geometry. Despite the chirality of this complex, only a single set of paramagnetic cross-peaks was observed for the protein. Fast exchange between the different propeller chiralities is not unexpected, as the exchange can proceed without dissociation of the DPA ligands from the lanthanide ion. 
Predicting the binding sites of DTPA or DPA complexes on proteins is not always straightforward. In the case of the IDA- $\mathrm{Cu}^{2+}$ complex, high selectivity for a single solventexposed histidine residue has been reported, allowing determination of distance restraints from PRE measurements [185]. Unfortunately, this approach is not available for lanthanide ions as their affinity for nitrogen ligands is very low.

In the case of $\left[\operatorname{Ln}(\mathrm{DPA})_{3}\right]^{3-}$ complexes bound to $\operatorname{ArgN}, \Delta \chi$-tensor fits located the complexes in the vicinity of positively charged amino acid side-chains [181]. Beyond electrostatic attraction, cation- $\pi$ interactions may contribute to the binding [186]. A comprehensive study of many proteins concluded that two basic residues (arginine or lysine) suffice to generate a binding site for $\left[\operatorname{Ln}(\mathrm{DPA})_{3}\right]^{3-}$, provided that no acidic side chains from aspartate or glutamate are nearby that could engage the arginine and lysine residues in salt bridges. In wild-type proteins, it is rare to find situations where two basic residues are nearby without either of them being able to engage in a salt bridge. Therefore, one or two mutations suffice to generate a binding site [183]. Notably, however, the magnitudes of the $\Delta \chi$ tensors obtained with $\left[\operatorname{Ln}(\mathrm{DPA})_{3}\right]^{3-}$ complexes were almost always much smaller than those obtained with covalently bound tags, which may in part be due to the flexibility of the long side chains of arginine and lysine. As the binding affinities of $\left[\operatorname{Ln}(\mathrm{DPA})_{3}\right]^{3-}$ complexes to proteins are weak (high micromolar to low millimolar range), it is not always possible to obtain 1:1 complexes with the target protein without also starting to populate alternative binding sites, although the chance of populating a single binding site is high if protein and DPA complex are used in equimolar ratio [181].

As PREs are effective regardless of lanthanide mobility, use of PREs generated by binding of $\left[\mathrm{Gd}(\mathrm{DPA})_{3}\right]^{3-}$ has been explored. $\mathrm{Gd}^{3+}$ generates much larger PREs than nitroxide radicals and there is no paramagnetic metal ion with isotropic $\chi$ tensor that would give PREs of intermediate size. However, scaling of PREs can be achieved by titration with $\left[\operatorname{Gd}(\mathrm{DPA})_{3}\right]^{3-}$. Due to the fast exchange between bound and free $\left[\operatorname{Gd}(\mathrm{DPA})_{3}\right]^{3-}$ complex, the PRE effects are correspondingly smaller with substoichiometric ratios of complex to protein than they are in the 1:1 complex with the paramagnetic agent [182]. [Gd(DPA $\left.)_{3}\right]^{3-}$ also binds to His6-tags or RAAAR peptide tags with submillimolar affinity, which can be used to elicit PREs [183].

A recent study analysed the effect of additional bromide, $-\mathrm{CO}_{2} \mathrm{H}$ or $-\mathrm{CH}_{2} \mathrm{OH}$ substituents at two different positions of the aromatic ring of the DPA molecule [184]. Using ubiquitin as the model protein, the lanthanide complexes formed with these DPA derivatives 
displayed different $\Delta \chi$-tensor magnitudes and orientations. The lowest $Q$-factor of the $\Delta \chi$ tensor fit was obtained with the asymmetric bromide derivative.

\subsubsection{Non-covalently binding protein tags}

Metal ions can also be attached to proteins by tags that bind non-covalently and achieve site-specificity by recognizing specific structural features of the protein. This strategy was first demonstrated using calmodulin loaded with four $\mathrm{Tb}^{3+}$ ions as the paramagnetic tag. Binding to a calmodulin binding peptide fused with the target protein was shown to generate significant RDCs in the target protein while the PCSs remained small [187]. The concept was extended in recent experiments that used the Erbin PDZ domain tagged with CLaNP-5 (12) to bind to the short peptide TGWETWV fused to ubiquitin or maltose binding protein. A single lanthanide attached to the PDZ domain was sufficient to generate significant RDCs and allowed fitting of the PCSs observed in the target protein by a single $\Delta \chi$ tensor [188]. The PDZ domain is fairly small (103 residues) and recognizes the fusion peptide with high affinity. Importantly, by using different double-cysteine mutants of the Erbin PDZ domain tagged with CLaNP-5, different molecular alignments of the same target protein were readily generated.

\subsection{Metal-binding unnatural amino acids}

Over 100 different unnatural amino acids can be incorporated into proteins by genetic encoding [189]. Some of these amino acids can bind metal ions. Unfortunately, the only unnatural amino acid that can bind lanthanides with adequate affinity is 2-amino-3-(8hydroxyquinolin-3-yl)propanoic acid (HQ-Ala), which has a side chain with an 8-hydroxyquinoline (8HQ) moiety. Many trials have shown that binding of lanthanides to solventexposed 8HQ residues results in quantitative protein precipitation [190], presumably because the coordination of the lanthanide is only bidentate, leaving too many coordination sites free to be occupied by other metal binding substituents from the protein.

\section{Generating PCSs with $\mathrm{Co}^{2+}$}

\section{1. $\mathrm{Co}^{2+}$ binding proteins}

$\mathrm{Co}^{2+}$-binding proteins are relatively rare, but zinc-binding proteins constitute a very large class of metalloproteins and often zinc can be replaced by $\mathrm{Co}^{2+}$ [191], allowing PCS measurements. Although the affinity of zinc fingers for $\mathrm{Co}^{2+}$ is about four orders of 
magnitude lower than for $\mathrm{Zn}^{2+}$, binding affinities in the nanomolar range can still be observed [192]. In an early example, PCSs were measured in this way for the transcription factor ADR1 that comprises a two-finger array of $\mathrm{His}_{2}-\mathrm{Cys}_{2}$ zinc fingers [193]. The $\Delta \chi$-tensor parameters of the $\mathrm{Co}^{2+}$ ions were assumed to be the same as those determined previously for a consensus His2-Cys2 zinc finger [194] and the observation of PCSs for an accessory polypeptide segment in the presence, but not the absence, of DNA was interpreted in terms of a disorder-to-order transition upon DNA binding [193]. Fitting $\Delta \chi$-tensors for both $\mathrm{Co}^{2+}$ centres from the single set of experimental PCSs would be challenging, as this would involve optimising a total of 16 tensor parameters. Substitution of $\mathrm{Zn}^{2+}$ by $\mathrm{Co}^{2+}$ in a Cys2-His-Cys zinc finger motif has also been shown to produce large PCSs [195].

In another example of zinc-binding proteins, bovine and human carbonic anhydrase II, large PCSs were observed in the $\mathrm{Co}^{2+}$-substituted enzymes. The magnitude of PCSs allowed determining the $\Delta \chi$ tensor using random coil chemical shifts as the diamagnetic reference and the tensor was interpreted in terms of metal coordination geometry [196, 197].

PCSs were similarly observed in the two-domain protein PA0128 from Pseudomonas aeruginosa by substituting a $\mathrm{Zn}^{2+}$ ion in the $\mathrm{N}$-terminal domain by a $\mathrm{Co}^{2+}$ ion. Although no $\Delta \chi$-tensor parameters could be determined, the PCSs generated by the $\mathrm{Co}^{2+}$ ion made it possible to interpret line-broadening effects in the C-terminal domain in terms of concerted domain motions [198].

As a final example, PCSs measured for the $\mathrm{Co}^{2+}$-substituted matrix metalloproteinase 12 (CoMMP-12), which naturally binds $\mathrm{Zn}^{2+}$, have been used to obtain structural information about neighbouring molecules in a single-crystal environment and refine the 3D structure of the protein $[199,200]$. The protein was used to show that nuclear spins in close proximity to the $\mathrm{Co}^{2+}$ ion become observable by solid-state NMR with ultrafast magic angle spinning [201]. A very recent study used these data to validate a novel multi-scale quantum-chemical approach for accurate PCS predictions in metalloproteins [202].

\section{2. $\mathrm{Co}^{2+}$ complexes generating sizeable PCSs}

Many lanthanide tags, for example EDTA, NTA and IDA tags, also bind $\mathrm{Co}^{2+}$ and $\mathrm{Zn}^{2+}$ (as the diamagnetic reference) very well. Tagging strategies using $\mathrm{Co}^{2+}$ as well as lanthanides have been discussed above. Unlike lanthanide ions, however, cobalt has a greater affinity for nitrogen ligands and binds more easily to bidentate ligands, which makes it possible to attach $\mathrm{Co}^{2+}$ ions to sites where lanthanides would not bind. An early example was 
the complex between the DNA octamer d(TTGGCCAA) 2 and chromomycin- $\mathrm{A}_{3}$, where $\mathrm{Co}^{2+}$ was bound to two oxygens of the chromophore [203, 204]. The measured PCSs greatly improved the quality of the NMR structure of the DNA-drug complex. PCSs restraints from $\mathrm{Co}^{2+}$ were also used to characterize complexes of helical peptides covalently connected to a cobalt-affine bipyridyl moiety [205]. Another early attempt used EDTA tag 4 attached to the E. coli arginine repressor [135]. As discussed in section 5.3.2.1, the racemic nature of the EDTA-Co ${ }^{2+}$ complex resulted in peak doubling arising from two sets of PCSs.

The IDA-tag 41 attached to two cysteine residues in an $i, i+4$ arrangement within the $\alpha$-helix of ubiquitin has been shown to lead to a hexadentate EDTA-like motif with $\mathrm{Co}^{2+}$, producing a good correlation between predicted and measured PCSs with a fairly large $\Delta \chi_{\mathrm{ax}}$ value of $-7.3 \times 10^{-32} \mathrm{~m}^{3}$ [206]. Recently, $\mathrm{Co}^{2+}$ bound to the cysteine-reactive 8 -HQ probe 45 was used to measure PCSs, RDCs and PREs in ubiquitin, and $\Delta \chi_{\text {ax }}$ values up to $-10.3 \times 10^{-32}$ $\mathrm{m}^{3}$ were reported [207]. While these magnitudes are respectable, larger paramagnetic effects can be obtained with lanthanide than with $\mathrm{Co}^{2+}$ ions. For example, a direct comparison between lanthanides and $\mathrm{Co}^{2+}$ using the $3 \mathrm{MDPA}$ tag (28) attached to $\operatorname{ArgN}$ gave $\Delta \chi_{\text {ax }}$ values of $-17.0 \times 10^{-32} \mathrm{~m}^{3}$ and $-4.6 \times 10^{-32} \mathrm{~m}^{3}$ for $\mathrm{Dy}^{3+}$ and $\mathrm{Co}^{2+}$, respectively [171]. An exceptionally large $\Delta \chi_{\mathrm{ax}}$ value of $12.6 \times 10^{-32} \mathrm{~m}^{3}$ has recently been reported for a special $\mathrm{Co}(\mathrm{II})$ clathrochelate compound dissolved in $\mathrm{CDCl}_{3}$ [208].

\subsection{Creating a $\mathrm{Co}^{2+}$ or $\mathrm{Mn}^{2+}$ binding site with an unnatural amino acid}

The first example of generating PCSs by directly binding a paramagnetic metal ion to an unnatural amino acid employed (2,2'-bipyridin-5-yl)-L-alanine (BpyAla) incorporated at three different sites of the West Nile virus NS2B-NS3 protease [209]. The amino acid binds $\mathrm{Co}^{2+}$, but for two of the mutants only PREs and no PCSs were observed, indicating conformational averaging of the PCSs due to side-chain flexibility. The third mutant produced PCSs that could be used to derive the corresponding $\Delta \chi$ tensor, suggesting that immobilization of the metal ion was aided by additional interaction with a histidine side chain of the protein.

More recently, HQ-Ala incorporated into two different membrane proteins in DHPC micelles has been shown to produce specific binding sites for $\mathrm{Mn}^{2+}$ which could be substituted by $\mathrm{Zn}^{2+}$ for PRE measurements [210]. In contrast to previous results with watersoluble proteins, metal ion binding did not trigger precipitation of the protein. 


\section{Macromolecular structure from pseudocontact shifts generated by lanthanide and cobalt tags}

\section{1. $3 D$ protein structure refinements using PCSs from lanthanides}

PCSs present structure restraints that provide highly useful information in $3 \mathrm{D}$ structure refinements, like numerous other, more traditional NMR parameters, such as nuclear Overhauser effects (NOE) and scalar coupling constants. Structure restraints from PCSs have been used to assist 3D structure refinements of metallo-proteins for over 20 years [89]. Structure refinement using PCSs from a metal tag (the EDTA tag 4 loaded with $\mathrm{Co}^{2+}$ ) was demonstrated in 2004, where the tag was attached to cysteine residues at three different sites [211].

With lanthanides bound to proteins, not only do large PCS effects (spanning over $40 \AA$ [12]) become available, but also significant RDCs. Therefore, lanthanides have been used in structure refinements not only to obtain PCSs but also RDCs. For example, a study of two different calmodulin-peptide complexes used the N60D mutant of calmodulin with three different paramagnetic lanthanides, to refine the corresponding crystal structures by PCSs and ${ }^{1} \mathrm{H}-{ }^{15} \mathrm{~N}$ RDCs. The results highlighted the much better fit of back-calculated to experimental data for PCSs than for RDCs prior to structure refinement [212]. This feature is readily explained by the relative insensitivity of PCSs to small changes in bond angles. PCSs are thus particularly helpful in structure refinements that aim for the global fold of the protein rather than fine structural detail.

A recent proof-of-principle study demonstrated that 3D protein structures can also be determined with the help of PCS data measured in the solid state using magic angle spinning (MAS). PCSs were measured of ${ }^{13} \mathrm{C}$ and backbone ${ }^{15} \mathrm{~N}$ spins of GB1 with the MMDPA (27) tag attached at three different sites, using $\mathrm{Co}^{2+}, \mathrm{Yb}^{3+}$, and $\mathrm{Tm}^{3+}$ ions to generate PCSs [213]. To avoid intermolecular PCSs, tagged GB1 was diluted 4 to 8-fold with untagged GB1. The root mean square deviation (RMSD) of the $\mathrm{C}^{\alpha}$ atoms was as low as $0.7 \AA$ with respect to the crystal structure. Solid-state MAS NMR has the advantage that solid-state signals are not subject to Curie spin relaxation [214], allowing observation of NMR signals of ligands that directly coordinate the paramagnetic metal ion [201].

Starting from a crystal structure, PCS data measured in solution have also been used to

judge whether a protein structure is conserved in solution. PCSs and ${ }^{1} \mathrm{H}_{-}{ }^{15} \mathrm{~N}$ RDCs of backbone amides were measured of a double-cysteine mutant of metalloproteinase MMP-1 
ligated with a CLaNP-5 tag (12) (loaded with $\mathrm{Tb}^{3+}, \mathrm{Tm}^{3+}$ or $\mathrm{Yb}^{3+}$ ) and the data were combined with the electron density observed in the crystal structure in order to determine a solution structure that fulfils the paramagnetic restraint while being closely similar to the crystal structure [215]. Very good agreement between experimental and back-calculated data was obtained for the refined structures, and the backbone RMSD between crystal and refined structures was lower than $0.4 \AA$. The same protocol applied to the N60D mutant of calmodulin complexed with DAPk peptide yielded less good fits.

Recently, PCSs generated by the tags $\mathbf{3 5}$ and $\mathbf{3 8}$ loaded with $\mathrm{Tm}^{3+}$ or $\mathrm{Tb}^{3+}$ and ligated to a cysteine residue in position 82 of $S$. aureus sortase A were used to determine the structures of loop segments of this protein in a transient intermediate [177]. More details are discussed in Section 7.4.5.

\subsection{D protein structure determinations using PCSs generated by lanthanide tags}

Going beyond structure refinement, PCSs can also be used for 3D structure determinations, if initial models of the protein can be built that allow fitting of $\Delta \chi$ tensors, as in the strategies implemented in the programs PCS-Rosetta and GPS-Rosetta [98, 99]. Although Rosetta can model the structures of small proteins without any experimental input beyond the amino-acid sequence [216], experimental restraints not only allow verification, but actually gain critical importance for determining the folds of proteins with more than about 120 residues. PCSs are uniquely suited in this context due to their long-range nature. Furthermore, PCSs of backbone amide protons can be measured in sensitive two-dimensional ${ }^{15} \mathrm{~N}-{ }^{1} \mathrm{H}$ correlation spectra, which present the best-resolved 2D NMR spectra of proteins.

While the concept of de novo protein structure determination (as opposed to structure refinement) exclusively from PCSs generated by lanthanide tags has been established, actual experimental examples are still rare. GPS-Rosetta was demonstrated using the C-terminal domain of ERp29, which is a construct of 127 residues [217]. The 3D structure was obtained using backbone amide PCSs generated from four different tagging sites using IDA-SH (40) or C1 (19a) tags loaded with either $\mathrm{Tb}^{3+}, \mathrm{Tm}^{3+}$ or $\mathrm{Y}^{3+}$ ions [99]. The resulting structure corrected the NMR structure determined previously from NOEs and scalar couplings [217]. Notably, the structure calculations converged well even though the PCS data were relatively sparse, with PCSs from more than a single tagging site observed for less than half the amino acid residues.

In contrast, it proved difficult to determine the fold of the 7-transmembrane-helix protein pSRII exclusively from PCS restraints derived from C2 (19b) lanthanide tags attached 
to four different positions of the protein, even though four different paramagnetic lanthanides were used $\left(\mathrm{Dy}^{3+}, \mathrm{Tb}^{3+}, \mathrm{Tm}^{3+}\right.$ or $\left.\mathrm{Yb}^{3+}\right)$ [218]. A total of 737 PCSs were measured for backbone amides and tryptophan side-chains, with at least one PCS assigned for two thirds of the residues. Assuming literature values for the $\Delta \chi$ tensors and imposing restraints for 132 backbone hydrogen bonds in the structure calculations, the backbone RMSD to the previously published NMR structure was $5 \AA$. It is unclear, how much the structure could have been improved by refining the $\Delta \chi$-tensor parameters during the structure computations. Ultimately, the RMSD to the target structure was reduced to $2.6 \AA$ by inclusion of over 200 NOEs, including a number of inter-helix NOEs, which by themselves were insufficient to determine the global fold of this protein.

Determining the 3D structure of another $\alpha$-helical protein, cytochrome $c^{\prime}$ from Rhodobacter capsulatus, also proved to be difficult from paramagnetic data only. Although the PCSs were complemented by paramagnetically induced RDCs, PREs and cross-correlated relaxation effects, the authors felt it necessary to define the helices by H-bond restraints [91]. Notably, however, the protein contained only a single paramagnetic centre $\left(\mathrm{Fe}^{2+}\right)$, which was associated with a relatively small $\Delta \chi$ tensor.

More recently, the GPS-Rosetta approach was used to determine the structure and location of the NS2B co-factor in the $27 \mathrm{kDa}$ NS2B/NS3 protease of serotype 2 dengue virus, using PCSs measured with $\mathrm{C} 1(\mathbf{1 9 a})$ and $\mathrm{C} 2(\mathbf{1 9 b})$ tags loaded with either $\mathrm{Tb}^{3+}, \mathrm{Tm}^{3+}$ or $\mathrm{Y}^{3+}$ ions at two different sites of NS3 [219]. While this example does not constitute a full structure determination, as the structure of NS3 was assumed to be the same as in previous crystal structures, the work demonstrated that PCSs elicited from two tagging sites can be sufficient to obtain correct folds of protein segments.

Recently, the structure of GB1 was determined with the help of backbone amide PCSs and ${ }^{1} \mathrm{H}_{-}{ }^{15} \mathrm{~N}$ RDCs by 2D in-cell NMR in frog oocytes [220]. GB1 was tagged at three different sites with the DOTA-M7Py tag loaded with $\mathrm{Tm}^{3+}$ or $\mathrm{Lu}^{3+}$ and GPS-Rosetta [99] was used for structure calculation. A different study used the related PyMTA tag loaded with $\mathrm{Tb}^{3+}$, $\mathrm{Tm}^{3+}$ or $\mathrm{Yb}^{3+}$ attached at two different sites of GB1 to determine its structure in frog oocytes, using GPS-Rosetta from PCSs of backbone amide protons only [221]. The Rosetta algorithm performs very well in computing the structures of small proteins such as GB1 without any protein-specific experimental data [213], so that these structure determinations can equally well be considered as structure refinements. 


\subsection{Structure determinations of protein-protein complexes using PCSs from lanthanides}

Intermolecular PCSs across a protein-protein interface deliver excellent restraints to define the $3 \mathrm{D}$ structure of the protein-protein complex, particularly in situations where the NMR resonances of interfacial residues are broadened by chemical exchange, or their chemical shifts change so much in the complex compared with the free proteins that new resonance assignments need to be established. The concept of this approach was established with a cytochrome $f$ - plastocyanin complex, where intermolecular PCSs generated by the heme $\mathrm{Fe}^{3+}$ ion in cytochrome $f$ allowed determination of the complex structure by rigid-body docking [222]. Subsequent work showed that for the corresponding complex from Prochlorothrix hollandica a significantly improved fit of the data could be obtained by assuming an ensemble of structures, as they may occur in an encounter complex [223].

This approach is greatly enhanced by lanthanide ions, due to larger PCSs and the available choice of different lanthanides, including very good diamagnetic references. In the case of the complex between the E. coli DNA polymerase III subunits $\varepsilon_{186}$ and $\theta$, lanthanide ions in the metal binding site of $\varepsilon_{186}$ induce significant PCSs in $\theta$ allowed determination of the $\varepsilon_{186}: \theta \quad$ rigid-body docking [109]. The study showed that ambiguities in the docking arrangement arising from the symmetry of the $\Delta \chi$ tensor of a single metal ion are lifted by the availability of PCSs from two different lanthanides $\left(\mathrm{Dy}^{3+}\right.$ and $\mathrm{Er}^{3+}$ in the case of the $\varepsilon_{186}: \theta$ ) .

The approach was first extended to non-metalloproteins by attaching a double-arm LBP [124] to one of the proteins in a protein-protein complex. The target protein was the p62 PB1 domain. The domain produces a front-to-back homo-oligomer, which was reduced to a dimer by suitable mutations. Combined with chemical shift perturbations, PCSs measured with four different lanthanide ions determined the structure of the $20 \mathrm{kDa}$ dimer [126].

In the same year (2010), a milestone study of the $65 \mathrm{kDa}$ complex of adrenoxin reductase (AdR) with adrenoxin illustrated the potential of the CLaNP-5 tag (12) for determining the structure of protein-protein complexes without resonance assignments of the tagged protein [224]. PCSs were generated in adrenoxin by Tm-CLaNP-5 tags attached at two different sites of AdR. Combined with PREs from a Gd-CLaNP-5 tag, chemical shift perturbations, and optimisation of the orientation of the $\Delta \chi$ tensors in an iterative fashion, the final rigid-body docking solutions computed with XPLOR-NIH showed RMSD values of no more than $3.2 \AA$ and were in agreement with the crystal structure of the complex. The study involved significant biochemical effort, as good labelling efficiencies were found for only two 
out of four tag attachment sites and an extensive mutation study was needed to identify the cysteine residues in wild-type AdR that had to be mutated prior to tag attachment. Notably, PCSs were observed over distances of up to $56 \AA$. Therefore, the structure could be determined despite the paramagnetic iron-sulfur cluster in AdR, which prevented observation of NMR signals near the protein-protein interface.

To solve the ambiguity of docking solutions arising from the symmetry of $\Delta \chi$ tensors, it was shown that the location and orientation of a $\Delta \chi$ tensor is readily varied by changing the length of the peptide linker in the fusion with a double-arm LBP [225]. The strategy was demonstrated with the complex between FKBP12-rapamycin and the mTOR FRB domain. Five different linker lengths were tested to identify two constructs that yielded different PCSs of good magnitude without perturbing the protein structure.

PCSs from single-arm lanthanide tags are more difficult to interpret if the tag is flexible. If a homology model of the 3D structure of the protein-protein complex is available, however, a single tag attached at a single site with a single paramagnetic lanthanide can yield conclusive experimental verification of the complex. This was demonstrated for the complex between the dengue virus NS2B-NS3 protease and aprotinin (BPTI), where an effective $\Delta \chi$ tensor established with inclusion of PCSs from residues near the site of interest proved sufficiently accurate to demonstrate that NS2B assumes the closed conformation throughout, including segments for which the crystal structure did not show electron density [69]. This study extended earlier work, where the C1 (19a) and C2 (19b) tags had been used with different lanthanides at three different sites to show that the protease also assumes the closed conformation in complex with a low-molecular weight inhibitor and, without inhibitor, after cleavage of the artificial peptide linker between NS2B and NS3 [161, 226].

\subsection{Lanthanide-generated PCSs for studies of dynamic proteins and protein-protein complexes}

If a molecule moves relative to the $\Delta \chi$ tensor of a paramagnetic metal ion, this will change the PCSs observed (unless the nuclear spins move along a PCS isosurface, which is unlikely for larger polypeptide segments). Therefore, PCSs from paramagnetic lanthanide tags attached to a structurally conserved part of the protein offer a unique tool to assess interdomain movements. In general, large-amplitude motions will reduce the magnitude of PCSs, as the spaces visited by the nuclear spins are likely characterized by PCSs of different sign. 


\subsubsection{PCSs of random coil peptides}

The reduction of PCS magnitude due to mobility is particularly apparent along a random coil polypeptide chain. An example is a study of the $166 \mathrm{kDa}$ complex between the $E$. coli DNA Pol III subunits $\alpha, \varepsilon$, and $\theta$. The $\varepsilon: \theta$ complex is structurally well defined, but it binds to $\alpha$ via a flexibly disordered polypeptide chain at the C-terminal end of $\varepsilon$. PCSs from a $\mathrm{Er}^{3+}, \mathrm{Tb}^{3+}$ or $\mathrm{Dy}^{3+}$ ion bound to the natural metal binding site in $\varepsilon$ decrease to very minor effects with increasing distance from the globular domain of $\varepsilon$ over about 15 residues of the flexible polypeptide segment [227]. Almost complete suppression of PCSs after about 15 residues of flexible polypeptide segment was also observed in the case of the long unstructured loop formed by the C-terminal domain of E. coli single-stranded DNA binding protein (SSB) [228].

A recent study attached the DOTA-M8 ytterbium tag to two different intrinsically disordered proteins, residues 454-526 of the protein "fused in sarcoma" and residues 1-101 of the lymphoid enhancer-binding factor 1 . Attached to cysteine residues engineered into the polypeptide chains, PCSs were observed between 5 and 25 residues from the tag attachment site, greatly improving spectral resolution and enabling unique resonance assignments. Remarkably, the PREs were sufficiently small to allow observation of the backbone amide resonances of the tagged cysteine residues [229].

These results are in overall agreement with expectations for a model that represents the polypeptide chain as a Gaussian chain of statistical chain segments [230], where a statistical chain segment corresponds to the polypeptide segment for which the relative orientation of the first and last amino acid residue is uncorrelated. After at most two statistical chain segments, this model would predict that amino acid residues sample spaces with opposite signs of PCS values. The length of the statistical chain segment in random-coil peptides corresponds to about nine amino acid residues [231].

\subsubsection{Inter-domain PCSs in multiple-domain proteins}

Likewise, inter-domain motions decrease the magnitude of PCSs. An early example was the N60D mutant of the two-domain protein calmodulin [232]. While $\mathrm{Tm}^{3+}$ and $\mathrm{Tb}^{3+}$ ions bound to the N-terminal domain generated significant PCSs in the N-terminal domain, the PCSs and paramagnetically induced ${ }^{1} \mathrm{H}^{15} \mathrm{~N}$ RDCs in the C-terminal domain were much smaller, and could be interpreted by an ensemble of three different relative domain orientations but not by a single one. The data subsequently formed the basis for maximum- 
occurrence calculations that assess the maximum contribution of a conformation in the ensemble [233-236]. In an alternative approach, the data were used to probe the conformational space of calmodulin by molecular dynamics and replica-averaged metadynamics simulations [237].

The N60D mutant of calmodulin was used further to study inter-domain mobility in a complex with a peptide derived from the voltage-gated calcium channel Cav1.2 (IQ), for which three different inter-domain orientations had been observed in the crystal. PCSs and RDCs of backbone amide protons generated by four different lanthanide ions (with two additional lanthanides used for cross-validation) were measured. Beyond inclusion of all three crystal conformations, a best ensemble fit of the data required four additional conformations derived from MD simulations [238].

With an increasing number of protein coordinates deposited in the protein data bank (PDB), there are also an increasing number of crystal structures of proteins in different conformations. PCSs present highly informative data on whether the conformations in solution match those in the crystal environment, and whether conformational changes occur in solution. One example is the dengue virus NS2B/NS3 protease, where crystal structures show very different conformations for NS2B, whereas PCSs measured in solution support only the closed conformation $[69,161,226]$. Another example is the E. coli aspartate/glutamate binding protein, where PCSs in the presence of glutamate agree with the crystal structure, whereas PCSs in the absence of glutamate indicate a more open conformation with a flexible hinge between the two domains [239]. The approach has also been used to decide whether T4 lysozyme without bound substrate or inhibitor assumes an open or closed conformation in solution [240]. The study showed that, based on data comprising paramagnetically induced ${ }^{1} \mathrm{H}-{ }^{15} \mathrm{~N}$ RDCs and PCSs from $\mathrm{Tb}^{3+}$ and $\mathrm{Tm}^{3+}$, an improved fit could be obtained by assuming an equilibrium between two different protein conformations, whereas inclusion of a third conformation no longer improved the fit. In the case of the glucose-galactose binding protein, MD simulations were used to generate transitions between open and closed conformations to explain PCSs and ${ }^{1} \mathrm{H}_{-}{ }^{15} \mathrm{~N}$ RDCs generated with a $\mathrm{Dy}^{3+}$ tag (10, Fig. 11) [241]. Again, the paramagnetic data could be explained by an equilibrium between two protein conformations.

Another recent study investigated the conformation of the cytochrome P450 CYP101A1 in the presence and absence of substrate (camphor) and the redox partner putidaredoxin [242]. Using the double-arm lanthanide tag CLaNP-7 (14) loaded with $\mathrm{Yb}^{3+}$ and attached at a single site, PCSs of backbone amides showed a better fit to a crystal structure in the closed conformation when substrate was present and to an open conformation 
in the absence of substrate. In the complex with both substrate and putidaredoxin, the PCSs observed for a P450 sample selectively labelled with ${ }^{15} \mathrm{~N}$-leucine were conserved, indicating the closed conformation in contrast to previous X-ray crystallographic and EPR results. Notably, the outstanding sensitivity of PCS measurements was critical to perform this study, as this $45 \mathrm{kDa}$ enzyme was only stable for a few hours in the absence of substrate.

It is common to derive crystal structures of protein-ligand complexes from crystals of apo-protein that are soaked with ligand molecules after crystallisation. This raises the possibility that crystal packing forces prevent the conformational changes that ligand molecules may induce in solution. In many instances, however, protein-ligand complexes resist crystallisation. For example, crystal structures of open and closed states have been reported of MurD, which is a three-domain $47 \mathrm{kDa}$ protein required for bacterial peptidoglycan (murein) biosynthesis, but the protein could not be crystallized together with ATP or stable analogues thereof. PCSs measured with Yb-CLaNP-5 showed that the binding of ATP- $\mathrm{Mg}^{2+}$ generates a semi-closed state that transitions into the fully closed state after binding of the UDP- $N$-acetylmuramoyl-L-alanine substrate and ATP hydrolysis [243].

\subsubsection{PCS studies of dynamic protein-protein complexes}

In principle, the long-range nature of PCSs generated by lanthanide tags also opens unique opportunities to study weak and dynamic protein-protein complexes, as the tags can be positioned sufficiently far from the interface to avoid steric interferences. Due to their distance dependence, however, intermolecular PCSs can be quite small and the RDCs resulting from paramagnetic alignment of the molecule in the magnetic field may be more sensitive reporters of dynamic protein-protein interactions. For example, the $23 \mathrm{kDa}$ complex between yeast cytochrome $c$ and bovine adrenodoxin is transient and structurally heterogeneous, as indicated by unexpectedly small RDCs observed in adrenodoxin, when a Yb-CLaNP-5 tag was attached to cytochrome $c$ [244]. In this example, the tag was too far from the adrenodoxin molecule to provide intermolecular PCSs of sufficient magnitude to be interpreted in terms of a structural ensemble.

In the case of the cytochrome P450cam-putidaredoxin complex, CLaNP-7 (14) loaded with $\mathrm{Yb}^{3+}$ or $\mathrm{Tm}^{3+}$ on two sites of P450cam and one site of putidaredoxin produced PCSs and RDCs that resulted in a structural model of the complex. In this case, evidence for a heterogeneous encounter state came primarily from PREs measured with Gd-CLaNP-7, which were larger than expected [245]. 
An interesting example of a dynamic protein-protein complex has recently been provided by the synaptotagmin- $1 \mathrm{C}_{2} \mathrm{~B}$ domain bound to the SNARE complex (total molecular weight $51 \mathrm{kDa}$ ) [246]. Using the C2-Dy ${ }^{3+}$ tag attached at two different sites of the SNARE complex yielded sizeable PCSs in the SNARE complex, but the PCSs observed for the $\mathrm{C}_{2} \mathrm{~B}$ domain were much smaller than expected, which was explained by a dynamic binding mode between the two proteins, arising from predominant interactions through charged rather than hydrophobic amino acid side chains. In this system only two out of ten different tagging sites tested delivered suitable PCSs.

\subsubsection{Relaxation dispersion and CEST experiments on protein signals shifted by PCSS}

A more difficult challenge is the use of paramagnetic metal ions to assist the analysis of conformational changes by relaxation dispersion experiments. Relaxation dispersion experiments are uniquely suited to revealing the existence of small populations of conformations with lifetimes on the micro- to millisecond time scale. It has been shown that paramagnetic lanthanide ions in the $\mathrm{Ca}^{2+}$ binding site of the $\mathrm{N}$-terminal domain of troponin $\mathrm{C}$ generate relaxation dispersion curves unobservable with $\mathrm{Ca}^{2+}$ or $\mathrm{La}^{3+}$ [247]. This effect was attributed primarily to motions in the lanthanide-binding site.

Similarly, substitution of the diamagnetic $\mathrm{Zn}^{2+}$ ion in the N-terminal domain of the two-domain protein PA0128 by a paramagnetic $\mathrm{Co}^{2+}$ ion produced relaxation dispersion curves for the C-terminal domain that were not observable in diamagnetic samples [198]. In this case, the effect was attributed to translational movements of the $\mathrm{C}$-terminal relative to the N-terminal domain.

Unfortunately, attempts to enhance relaxation dispersion effects by paramagnetic lanthanide tags have so far been thwarted by difficulties distinguishing motions of the protein from those of the tag $[151,152,198]$. Alternatively, however, chemical exchange saturation transfer (CEST) experiments can be used to measure chemical shifts and PCSs of lowpopulated states. This has recently been demonstrated for the Abp1p SH3 domain tagged with 4MMPyMTA (33). In the presence of 3\% Ark1p peptide, ${ }^{15} \mathrm{~N}$ PCSs of five residues measured with $\mathrm{Tb}^{3+}$ were shown to agree between the excited state of apo-Abp1p SH3 domain and its complex with peptide [175]. In the same work, a mutant of the HYPA/FBP11 FF domain was ligated with the same $\mathrm{Tb}^{3+}$ tag. This domain is in exchange with a small population of unfolded protein, which was confirmed by the small magnitude of the PCSs observed for seven backbone amides in ${ }^{15} \mathrm{~N}$ CEST experiments. 


\subsubsection{PCSs for 3D structure determination of short-lived enzyme intermediates}

Two-dimensional HSQC spectra can be measured very quickly, allowing correspondingly rapid collection of structural restraints from PCSs. This has recently been exploited to determine the conformation of a short-lived enzyme intermediate. A cysteine mutant of $S$. aureus sortase A was used to attach the tags $\mathbf{3 8}$ or $\mathbf{3 5}$ and measure PCSs of backbone amides by ${ }^{15} \mathrm{~N}-\mathrm{HSQC}$ spectra of samples right after addition of substrate peptide. Different PCSs were observed for a number of loop segments in the resulting transient thioester intermediate to those in the free enzyme. The PCSs generated by $\mathrm{Dy}^{3+}, \mathrm{Tb}^{3+}$ and $\mathrm{Tm}^{3+}$ were used to calculate the structure of the enzyme in the intermediate, assuming that the structure of segments with conserved PCSs did not change from that of the free enzyme [177].

\subsection{Protein-small molecule interactions}

\subsubsection{Studies with lanthanide tags on the protein}

There is much scope for using PCSs to study the binding site, orientation and structure of ligand molecules binding to proteins, in particular as paramagnetic effects can be elicited by tags that are quite remote from the ligand binding site. PCSs generated by lanthanide ions are particularly suitable, as the paramagnetic shifts are almost exclusively due to pseudocontact shifts, although it has been shown that contact shifts can be taken into account for paramagnetic effects that are generated by transition metal ions such as $\mathrm{Fe}^{2+}, \mathrm{Co}^{2+}$ or $\mathrm{Ni}^{2+}$ located in the vicinity of the ligand [248].

In general, proteins have been tagged with lanthanides at one or several sites. Some studies also explored the alternative approach of attaching lanthanide tags to ligands (see also Section 5.3.1).

The first published example where PCSs from lanthanides were used to define the exact binding mode of a ligand was a study of the affinity and binding mode of thymidine in the active site of the E. coli DNA polymerase III subunit $\varepsilon_{186}$. This protein is a proof-reading exonuclease that forms a tight complex with the subunit $\theta$ [249]. The metal ions in the active site were replaced by $\mathrm{Tb}^{3+}, \mathrm{Dy}^{3+}$ or $\mathrm{Er}^{3+}$. PCSs were measured in one-dimensional NMR spectra of the thymidine ligand. With a dissociation constant of about $7 \mathrm{mM}$, the ligand binds only weakly and is in fast exchange between the bound and free states; the NMR signals of the ligand are therefore averaged between the two states, reducing the magnitude of the PCSs in proportion to the fraction bound. Even with a 550-fold excess of ligand, PCSs were still measurable because the distance between lanthanide and ligand binding site was short. Straightforward $1 \mathrm{D}{ }^{1} \mathrm{H}$ - and ${ }^{13} \mathrm{C}-\mathrm{NMR}$ spectra were sufficient to measure the PCSs induced by 
$\mathrm{Tb}^{3+}, \mathrm{Dy}^{3+}$ and $\mathrm{Er}^{3+}$. Combined with the $\Delta \chi$ tensors determined from the ${ }^{15} \mathrm{~N}-\mathrm{HSQC}$ spectrum of the protein, the PCSs determined the binding affinity and orientation of the thymidine ligand with respect to the $\varepsilon_{186}$ subunit, which proved to be closely related to the binding mode observed for thymidine monophosphate by X-ray crystallography.

Another early study analysed the weak binding of lactose to galactin-3 using paramagnetic restraints from an LBP fused to the protein [250]. The measurements used a 5:1 ratio of ligand to protein, which was found to be the optimal compromise between signal-tonoise ratio and the size of PCSs and RDCs in the ligand, measured in $\left[{ }^{13} \mathrm{C}-{ }^{1} \mathrm{H}\right]-\mathrm{HSQC}$ spectra with $\mathrm{Dy}^{3+}$ as the paramagnetic ion. These data alone produced a structure having an RMSD of $4.3 \AA$ for the ligand atoms relative to the crystal structure, which decreased to $1.9 \AA$ with the inclusion of a single intermolecular NOE in the calculation.

Using a LBP fused to the N-terminus of the growth factor receptor-bound protein 2 (Grb2) SH2 domain and anchored to the protein at a second point via a disulfide bond, the binding modes of two ligands of different affinity were determined by PCSs from four different paramagnetic lanthanide ions [125]. Deuterated protein was used to observe the ligand signals in $1 \mathrm{D}{ }^{1} \mathrm{H}-\mathrm{NMR}$ experiments. The ligand conformers and positions determined from the PCS data displayed RMSD values between 1.9 and $3 \AA$, indicating that PCS data from a single lanthanide site can leave substantial structural uncertainties in flexible ligand molecules. It was noted that loading the LBP tag on the protein with $\mathrm{Gd}^{3+}$ delivered a sensitive way of screening a library of ligands for binding by using PREs.

If the binding mode of a ligand has been determined by intermolecular NOEs, PCSs from a single tagging site provide valuable restraints for cross-validation. In this way, the binding mode of ${ }^{13} \mathrm{C} /{ }^{15} \mathrm{~N}$ labelled dGMP to the R3H domain of human $\mathrm{S} \mu \mathrm{bp}-2$, as well as the protein conformation itself, were confirmed by PCSs using the $\mathrm{C} 1$ tag (19a) loaded with $\mathrm{Tb}^{3+}$ or $\mathrm{Yb}^{3+}[251]$.

The value of PCSs generated from tags positioned at three different sites of the target protein was explored with the complex of human FKB12, tagged with $\mathrm{Yb}^{3+}$-CLaNP-5 (12), with a weakly binding small molecule [67]. A 1.3-fold excess of ligand was sufficient to allow selective detection of the ligand signals in $1 \mathrm{D}{ }^{1} \mathrm{H}-\mathrm{NMR}$ spectra, with the help of a $T_{2}$ relaxation filter to suppress the protein signals. At this ligand excess, only $2 \%$ of the ligand molecules were in the bound state and the PCSs observed in the ligand were less than $10 \mathrm{~Hz}$. An RMSD of $2.8 \AA$ was obtained between ligand coordinates determined from PCSs and ligand coordinates determined from intermolecular NOEs, but it was noted that the NOE structure matched the PCS data less well than the PCS-based docking model. It was noted that 
PCSs from more than three tagging sites may be required for unambiguous identification of a ligand binding site, unless additional information from sources other than PCSs is available. (A single PCS restricts the position of a nuclear spin to a PCS isosurface. A second PCS from a different tagging site locates the spin on the line formed by the intersection between two PCS isosurfaces. With the PCS isosurface from a third tagging site, this line intersects with the third isosurface at only two points. Usually, only one of these two points is compatible with independent chemical information such as van der Waals contacts. Otherwise, the ambiguity can be resolved by the PCS from a fourth tagging site.)

NMR studies of protein-ligand complexes are greatly facilitated if the ligand is in fast exchange between the bound and free state, which facilitates the observation of the ligand resonances, as the ligand can be added in excess and the properties of the bound ligand derived from the average NMR spectrum. Observing the NMR spectrum of a non-exchanging ligand is much harder, as the bound-state ligand signals must be identified in a 1:1 complex with the protein. This problem has recently been addressed by using a ligand containing a tert-butyl group, which produces an exceptionally narrow and intense signal for which NOEs can readily be observed. This approach has been demonstrated with the dengue virus NS2B/NS3 protease in complex with a tert-butyl-group-containing inhibitor that forms a covalent bond to the active-site serine residue [252]. From cross-peaks observed in 2D NOESY spectra, PCSs could readily be measured not only for the tert-butyl group, but also for its NOE partners, for protease samples labelled at three different sites with C2 tags (19b) loaded with $\mathrm{Tb}^{3+}$ or $\mathrm{Tm}^{3+}$. Using the $\Delta \chi$ tensors determined from PCSs of backbone amides of the protein, the PCSs measured for the bound ligand defined localisation spaces on the protein surface, which indicated where the tert-butyl group and each of its NOE partners are located. Finally, modelling software was used to determine structural details of those parts of the ligand for which no PCSs could be measured.

\subsubsection{Studies with lanthanide or cobalt tag on the ligand}

Three different examples of ligand-protein studies using lanthanide-tagged ligands instead of tagged proteins have recently been reported. The interactions studied were of lactose with galectin-3 [82], fucose with a viral coat protein [131], and sevoflurane with calmodulin [132]. All three examples, described in more detail in Section 5.3.1, explored weak intermolecular interactions, where residual tag flexibility compromises the value of PCSs for gaining detailed structural information about specific intermolecular interactions. Furthermore, it is difficult to attach lanthanides at more than a single site of a small ligand, 
leaving ambiguities in PCS interpretation even if ligand and tag are perfectly immobilized on the target protein.

An interesting hybrid strategy is to generate PCSs in a ligand by attaching a metal tag to another ligand that binds tightly to the target protein, resulting in a secondary site screening approach. This was demonstrated with the N-terminal coiled coil of HIV-1 gp41 as the target, a 39-residue $\alpha$-helical peptide labelled with an EDTA-Co ${ }^{2+}$ complex at an $\mathrm{N}$-terminal cysteine residue as the tag, and 3-p-fluorobenzylbenzoic acid as the ligand binding to the target with low affinity [253]. Although PCSs were observable for only six ligand protons, and their values were small $(<10 \mathrm{~Hz}), \Delta \chi$-tensor fits allowed discrimination between different ligand poses.

\section{Conclusion and prospects}

With the coordinates of tens of thousands of proteins deposited in the protein data bank, the groundwork has been laid for many more investigations of protein conformational change, which ultimately is at the heart of the function of most proteins, ranging from small local changes in enzyme catalysis and ligand binding to large-amplitude conformational changes in signalling and motor proteins. By choosing the attachment sites of paramagnetic tags, it is possible to study such protein properties site-selectively without having to solve again the 3D structure of the entire protein, as required in X-ray crystallography or cryoelectron microscopy. In particular, owing to the long-range nature of paramagnetic effects, lanthanide tags lend themselves to site-specific studies where the tag must be sufficiently far from the site of interest not to interfere. To support site-specific NMR studies, it will be useful to develop improved methods for labelling the site of interest with NMR isotopes, e.g. by segmental isotopic labelling [254] or residue-specific labelling [255, 256].

In view of the general importance of NMR spectroscopy in drug development, the availability of protein crystal structures also provides fertile ground for further developments of lanthanide-tagging techniques with the aim of fragment-based drug design.

In closing, we note an additional application of paramagnetic lanthanide tags that is emerging in EPR spectroscopy, where distances between two gadolinium ions can be measured over ranges of 20 to $80 \AA$ in frozen solutions [257]. The high sensitivity of EPR spectroscopy may allow such experiments in cells at physiological concentrations. For these applications, it is important that the $\mathrm{Gd}^{3+}$ ion does not leak from the tag and that the tag is 
rigidly attached to the protein in order to generate narrow distance distributions. Further tag development thus has an importance that goes beyond its applications in NMR spectroscopy.

\section{Acknowledgements}

C. N. thanks the Alexander von Humboldt Foundation for a Feodor Lynen fellowship. Financial support by the Australian Research Council is gratefully acknowledged.

\section{References}

[1] D.F. Hansen, W.M. Westler, M.B. Kunze, J.L. Markley, F. Weinhold, J.J. Led, Accurate structure and dynamics of the metal-site of paramagnetic metalloproteins from NMR parameters using natural bond orbitals, J. Am. Chem. Soc. 134 (2012) 4670-4682.

[2] G.T. Charnock, I. Kuprov, A partial differential equation for pseudocontact shift, Phys. Chem. Chem. Phys. 16 (2014) 20184-20189.

[3] P. Fernández, H. Pritzkow, J.J. Carbó, P. Hofmann, M. Enders, ${ }^{1} \mathrm{H}$ NMR investigation of paramagnetic chromium(III) olefin polymerization catalysts: Experimental results, shift assignment and prediction by quantum chemical calculations, Organometallics 26 (2007) 4402-4412.

[4] I. Bertini, C. Luchinat, NMR of Paramagnetic Molecules in Biological Systems, Benjamin/Cummings Publishing Co., Menlo Park, New York, 1986.

[5] G. Pintacuda, K. Hohenthanner, G. Otting, N. Müller, Angular dependence of dipoledipole-Curie-spin cross-correlation effects in high-spin and low-spin paramagnetic myoglobin, J. Biomol. NMR 27 (2003) 115-132.

[6] T.J. Carruthers, P.D. Carr, C.T. Loh, C.J. Jackson, G. Otting, Iron(III) located in the dinuclear metallo-beta-lactamase IMP-1 by pseudocontact shifts, Angew. Chem. Int. Ed. 53 (2014) 14269-14272.

[7] F. Rastrelli, A. Bagno, Predicting the ${ }^{1} \mathrm{H}$ and ${ }^{13} \mathrm{C}$ NMR spectra of paramagnetic $\mathrm{Ru}(\mathrm{III})$ complexes by DFT, Magn. Reson. Chem. 48 Suppl 1 (2010) S132-S141.

[8] M.R. Jensen, J.J. Led, Metal-protein interactions: Structure information from $\mathrm{Ni}^{2+}$-induced pseudocontact shifts in a native nonmetalloprotein, Biochemistry 45 (2006) 8782-8787.

[9] A. Donaire, J. Salgado, J.M. Moratal, Determination of the magnetic axes of cobalt(II) and nickel(II) azurins from ${ }^{1} \mathrm{H}$ NMR data: influence of the metal and axial ligands on the origin of magnetic anisotropy in blue copper proteins, Biochemistry 37 (1998) 8659-8673.

[10] L.W. Donaldson, N.R. Skrynnikov, W.Y. Choy, D.R. Muhandiram, B. Sarkar, J.D. Forman-Kay, L.E. Kay, Structural characterization of proteins with an attached ATCUN motif by paramagnetic relaxation enhancement NMR spectroscopy, J. Am. Chem. Soc. 123 (2001) 9843-9847.

[11] M.R. Jensen, C. Lauritzen, S.W. Dahl, J. Pedersen, J.J. Led, Binding ability of a HHPtagged protein towards $\mathrm{Ni}^{2+}$ studied by paramagnetic NMR relaxation: The possibility of obtaining long-range structure information, J. Biomol. NMR 29 (2004) 175-185.

[12] M. Allegrozzi, I. Bertini, M.B.L. Janik, Y.-M. Lee, G. Liu, C. Luchinat, Lanthanideinduced pseudocontact shifts for solution structure refinements of macromolecules in shells up to $40 \AA$ from the metal ion, J. Am. Chem. Soc. 122 (2000) 4154-4161.

[13] M.J. Schmidt, A. Fedoseev, D. Bücker, J. Borbas, C. Peter, M. Drescher, D. Summerer, EPR distance measurements in native proteins with genetically encoded spin labels, ACS Chem. Biol. 10 (2015) 2764-2771. 
[14] H. Irving, R.J.P. Williams, The stability of transition-metal complexes, J. Chem. Soc. (1953) 3192-3210.

[15] I. Bertini, C. Luchinat, G. Parigi, Magnetic susceptibility in paramagnetic NMR, Prog. NMR Spectrosc. 40 (2002) 249-273.

[16] L. Banci, I. Bertini, C. Luchinat, Nuclear and Electron Relaxation, Verlag Chemie, Berlin, 1991.

[17] I. Bertini, C. Luchinat, G. Parigi, Solution NMR of Paramagnetic Molecules, Volume 2: Applications to Metallobiomolecules and Models, Elsevier, Amsterdam, 2001.

[18] J. Koehler, J. Meiler, Expanding the utility of NMR restraints with paramagnetic compounds: Background and practical aspects, Prog. NMR Spectrosc. 59 (2011) 360-389.

[19] G. Otting, Protein NMR using paramagnetic ions, Annu. Rev. Biophys. 39 (2010) 387405.

[20] M. Piccioli, P. Turano, Transient iron coordination sites in proteins: Exploiting the dual nature of paramagnetic NMR, Coord. Chem. Rev. 284 (2015) 313-328.

[21] I. Bertini, C. Luchinat, New applications of paramagnetic NMR in chemical biology, Curr. Opin. Chem. Biol. 3 (1999) 145-151.

[22] I. Bertini, C. Luchinat, G. Parigi, R. Pierattelli, NMR spectroscopy of paramagnetic metalloproteins, ChemBioChem 6 (2005) 1536-1549.

[23] I. Bertini, C. Luchinat, G. Parigi, R. Pierattelli, Perspectives in paramagnetic NMR of metalloproteins, Dalton Trans. (2008) 3782-3790.

[24] M. Ubbink, J.A. Worrall, G.W. Canters, E.J. Groenen, M. Huber, Paramagnetic resonance of biological metal centers, Annu. Rev. Biophys. Biomol. Struct. 31 (2002) 393422.

[25] F. Arnesano, L. Banci, M. Piccioli, NMR structures of paramagnetic metalloproteins, Q. Rev. Biophys. 38 (2005) 167-219.

[26] K. Kato, T. Yamaguchi, Paramagnetic NMR probes for characterization of the dynamic conformations and interactions of oligosaccharides, Glycoconj. J. 32 (2015) 1-9.

[27] M.J. Knight, I.C. Felli, R. Pierattelli, L. Emsley, G. Pintacuda, Magic angle spinning NMR of paramagnetic proteins, Acc. Chem. Res. 46 (2013) 2108-2116.

[28] I. Sengupta, P.S. Nadaud, C.P. Jaroniec, Protein structure determination with paramagnetic solid-state NMR spectroscopy, Acc. Chem. Res. 46 (2013) 2117-2126.

[29] A. Bhaumik, C. Luchinat, G. Parigi, E. Ravera, M. Rinaldelli, NMR crystallography on paramagnetic systems: solved and open issues, CrystEngComm 15 (2013) 8639-8656.

[30] G.M. Clore, J. Iwahara, Theory, practice, and applications of paramagnetic relaxation enhancement for the characterization of transient low-population states of biological macromolecules and their complexes, Chem. Rev. 109 (2009) 4108-4139.

[31] M.A. Hass, M. Ubbink, Structure determination of protein-protein complexes with longrange anisotropic paramagnetic NMR restraints, Curr. Opin. Struct. Biol. 24 (2014) 45-53.

[32] M. John, G. Otting, Strategies for measurements of pseudocontact shifts in protein NMR spectroscopy, ChemPhysChem 8 (2007) 2309-2313.

[33] J.H. Prestegard, D.A. Agard, K.W. Moremen, L.A. Lavery, L.C. Morris, K. Pederson, Sparse labeling of proteins: Structural characterization from long range constraints, J. Magn. Reson. 241 (2014) 32-40.

[34] P.H.J. Keizers, M. Ubbink, Paramagnetic tagging for protein structure and dynamics analysis, Prog. NMR Spectrosc. 58 (2011) 88-96.

[35] F. Rodriguez-Castañeda, P. Haberz, A. Leonov, C. Griesinger, Paramagnetic tagging of diamagnetic proteins for solution NMR, Mag. Res. Chem. 44 (2006) S10-S16.

[36] X.-C. Su, G. Otting, Paramagnetic labelling of proteins and oligonucleotides for NMR, J. Biomol. NMR 46 (2010) 101-112. 
[37] X.-C. Su, G. Otting, Erratum to: Paramagnetic labelling of proteins and oligonucleotides for NMR, J. Biomol. NMR 50 (2011) 99-100.

[38] M. Fragai, C. Luchinat, G. Parigi, E. Ravera, Conformational freedom of metalloproteins revealed by paramagnetism-assisted NMR, Coord. Chem. Rev. 257 (2013) 2652-2667.

[39] A. Carlon, E. Ravera, W. Andrałojć, G. Parigi, G.N. Murshudov, C. Luchinat, How to tackle protein structural data from solution and solid state: an integrated approach, Prog. NMR Spectrosc. 92-93 (2016) 54-70.

[40] W.-M. Liu, M. Overhand, M. Ubbink, The application of paramagnetic lanthanoid ions in NMR spectroscopy on proteins, Coord. Chem. Rev. 273-274 (2014) 2-12.

[41] G. Otting, Prospects for lanthanides in structural biology by NMR, J. Biomol. NMR 42 (2008) $1-9$.

[42] G. Pintacuda, M. John, X.-C. Su, G. Otting, NMR structure determination of proteinligand complexes by lanthanide labeling, Acc. Chem. Res. 40 (2007) 206-212.

[43] K.N. Allen, B. Imperiali, Lanthanide-tagged proteins - an illuminating partnership, Curr. Opin. Chem. Biol. 14 (2010) 247-254.

[44] D. Parker, R.S. Dickins, H. Puschmann, C. Crossland, J.A. Howard, Being excited by lanthanide coordination complexes: aqua species, chirality, excited-state chemistry, and exchange dynamics, Chem. Rev. 102 (2002) 1977-2010.

[45] G.M. Clore, A.M. Gronenborn, A. Bax, A robust method for determining the magnitude of the fully asymmetric alignment tensor of oriented macromolecules in the absence of structural information, J. Magn. Reson. 133 (1998) 216-221.

[46] C. Schmitz, M.J. Stanton-Cook, X.-C. Su, G. Otting, T. Huber, Numbat: an interactive software tool for fitting $\Delta \chi$-tensors to molecular coordinates using pseudocontact shifts, J. Biomol. NMR 41 (2008) 179-189.

[47] C. Schmitz, M. John, A.Y. Park, N.E. Dixon, G. Otting, G. Pintacuda, T. Huber, Efficient $\chi$-tensor determination and $\mathrm{NH}$ assignment of paramagnetic proteins, J. Biomol. NMR 35 (2006) 79-87.

[48] P.H. Keizers, A. Saragliadis, Y. Hiruma, M. Overhand, M. Ubbink, Design, synthesis, and evaluation of a lanthanide chelating protein probe: CLaNP-5 yields predictable paramagnetic effects independent of environment, J. Am. Chem. Soc. 130 (2008) 1480214812.

[49] I. Bertini, M.B. Janik, Y.M. Lee, C. Luchinat, A. Rosato, Magnetic susceptibility tensor anisotropies for a lanthanide ion series in a fixed protein matrix, J. Am. Chem. Soc. 123 (2001) 4181-4188.

[50] B.M. Alsaadi, F.J.C. Rossotti, R.J.P. Williams, Electron relaxation rates of lanthanide aquo-cations, J. Chem. Soc., Dalton Trans. (1980) 2147-2150.

[51] I. Solomon, Relaxation processes in a system of two spins, Phys. Rev. 99 (1955) 559565.

[52] M. Guéron, Nuclear relaxation in macromolecules by paramagnetic ions: A novel mechanism, J. Magn. Reson. 19 (1975) 58-66.

[53] G. Pintacuda, A. Kaikkonen, G. Otting, Modulation of the distance dependence of paramagnetic relaxation enhancements by CSA x DSA cross-correlation, J. Magn. Reson. 171 (2004) 233-243.

[54] I. Bertini, C. Luchinat, D. Tarchi, Are true scalar proton-proton connectivities ever measured in COSY spectra of paramagnetic macromolecules? Chem. Phys. Lett. 203 (1993) 445-449.

[55] R. Ghose, J.H. Prestegard, Electron spin-nuclear spin cross-correlation effects on multiplet splittings in paramagnetic proteins, J. Magn. Reson. 128 (1997) 138-143.

[56] A.J. Vega, D. Fiat, Nuclear relaxation processes of paramagnetic complexes. The slowmotion case, Mol. Phys. 31 (1976) 347-355. 
[57] I. Bertini, J. Kowalewski, C. Luchinat, G. Parigi, Cross correlation between the dipoledipole interaction and the Curie spin relaxation: the effect of anisotropic magnetic susceptibility, J. Magn. Reson. 152 (2001) 103-108.

[58] J. Boisbouvier, P. Gans, M. Blackledge, B. Brutscher, D. Marion, Long-range structural information in NMR studies of paramagnetic molecules from electron spin-nuclear spin cross-correlated relaxation, J. Am. Chem. Soc. 121 (1999) 7700-7701.

[59] P.K. Madhu, R. Grandori, K. Hohenthanner, P.K. Mandal, N. Müller, Geometry dependent two-dimensional heteronuclear multiplet effects in paramagnetic proteins, J. Biomol. NMR 20 (2001) 31-37.

[60] I. Bertini, G. Cavallaro, M. Cosenza, R. Kümmerle, C. Luchinat, M. Piccioli, L. Poggi, Cross correlation rates between Curie spin and dipole-dipole relaxation in paramagnetic proteins: the case of cerium substituted calbindin D $_{9 k}$, J. Biomol. NMR 23 (2002) 115-125.

[61] F. Kateb, M. Piccioli, New routes to the detection of relaxation allowed coherence transfer in paramagnetic molecules, J. Am. Chem. Soc. 125 (2003) 14978-14979.

[62] H.W. Orton, I. Kuprov, C.-T. Loh, G. Otting, Using paramagnetism to slow down nuclear relaxation in protein NMR, J. Phys. Chem. Lett. (2016) 10.1021/acs.jpclett.6b02417.

[63] B.D. Nguyen, Z. Xia, D.C. Yeh, K. Vyas, H. Deaguero, G.N. La Mar, Solution NMR determination of the anisotropy and orientation of the paramagnetic susceptibility tensor as a function of temperature for metmyoglobin cyanide: Implications for the population of excited electronic states, J. Am. Chem. Soc. 121 (1999) 208-217.

[64] C.N. Reilley, B.W. Good, R.D. Allendoerfer, Separation of contact and dipolar lanthanide induced nuclear magnetic resonance shifts: evaluation and application of some structure independent methods, Anal. Chem. 48 (1976) 1446-1458.

[65] A.D. Sherry, P.P. Yang, L.O. Morgan, Separation of contact and pseudocontact contributions to ${ }^{13} \mathrm{C}$ lanthanide induced shifts in non-axially-symmetric lanthanide ethylenediaminetetraacetate chelates, J. Am. Chem. Soc. 102 (1980) 5755-5759.

[66] Q. Bashir, A.N. Volkov, G.M. Ullmann, M. Ubbink, Visualization of the encounter ensemble of the transient electron transfer complex of cytochrome $c$ and cytochrome $c$ peroxidase, J. Am. Chem. Soc. 132 (2010) 241-247.

[67] J.Y. Guan, P.H. Keizers, W.M. Liu, F. Löhr, S.P. Skinner, E.A. Heeneman, H. Schwalbe, M. Ubbink, G. Siegal, Small-molecule binding sites on proteins established by paramagnetic NMR spectroscopy, J. Am. Chem. Soc. 135 (2013) 5859-5868.

[68] D. Shishmarev, G. Otting, How reliable are pseudocontact shifts induced in proteins and ligands by mobile paramagnetic metal tags? A modelling study, J. Biomol. NMR 56 (2013) 203-216.

[69] W.N. Chen, K.V. Loscha, C. Nitsche, B. Graham, G. Otting, The dengue virus NS2BNS3 protease retains the closed conformation in the complex with BPTI, FEBS Lett. 588 (2014) 2206-2211.

[70] M. John, M.J. Headlam, N.E. Dixon, G. Otting, Assignment of paramagnetic ${ }^{15} \mathrm{~N}-\mathrm{HSQC}$ spectra by heteronuclear exchange spectroscopy, J. Biomol. NMR 37 (2007) 43-51.

[71] M. John, A.Y. Park, N.E. Dixon, G. Otting, NMR detection of protein ${ }^{15} \mathrm{~N}$ spins near paramagnetic lanthanide ions, J. Am. Chem. Soc. 129 (2007) 462-463.

[72] M. John, C. Schmitz, A.Y. Park, N.E. Dixon, T. Huber, G. Otting, Sequence-specific and stereospecific assignment of methyl groups using paramagnetic lanthanides, J. Am. Chem. Soc. 129 (2007) 13749-13757.

[73] J.D. Swarbrick, P. Ung, S. Chhabra, B. Graham, An iminodiacetic acid based lanthanide binding tag for paramagnetic exchange NMR spectroscopy, Angew. Chem. Int. Ed. 50 (2011) 4403-4406.

[74] H. Yagi, A. Maleckis, G. Otting, A systematic study of labelling an $\alpha$-helix in a protein with a lanthanide using IDA-SH or NTA-SH tags, J. Biomol. NMR 55 (2013) 157-166. 
[75] G. Pintacuda, M.A. Keniry, T. Huber, A.Y. Park, N.E. Dixon, G. Otting, Fast structurebased assignment of ${ }^{15} \mathrm{~N}$ HSQC spectra of selectively ${ }^{15} \mathrm{~N}$-labeled paramagnetic proteins, J. Am. Chem. Soc. 126 (2004) 2963-2970.

[76] C. Cao, J.L. Chen, Y. Yang, F. Huang, G. Otting, X.-C. Su, Selective ${ }^{15}$ N-labeling of the side-chain amide groups of asparagine and glutamine for applications in paramagnetic NMR spectroscopy, J. Biomol. NMR 59 (2014) 251-261.

[77] W. Bermel, I. Bertini, I.C. Felli, M. Piccioli, R. Pierattelli, ${ }^{13} \mathrm{C}$-detected protonless NMR spectroscopy of proteins in solution, Prog. NMR Spectrosc. 48 (2006) 25-45.

[78] M. Gochin, Nuclear magnetic resonance characterization of a paramagnetic DNA-drug complex with high spin cobalt; assignment of the ${ }^{1} \mathrm{H}$ and ${ }^{31} \mathrm{P}$ NMR spectra, and determination of electronic, spectroscopic and molecular properties, J. Biomol. NMR 12 (1998) 243-257.

[79] I.I. Bertini, I.C. Felli, C. Luchinat, High magnetic field consequences on the NMR hyperfine shifts in solution, J. Magn. Reson. 134 (1998) 360-364.

[80] J.R. Tolman, J.M. Flanagan, M.A. Kennedy, J.H. Prestegard, Nuclear magnetic dipole interactions in field-oriented proteins: Information for structure determination in solution, Proc. Natl. Acad. Sci. USA 92 (1995) 9279-9283.

[81] M. John, A.Y. Park, G. Pintacuda, N.E. Dixon, G. Otting, Weak alignment of paramagnetic proteins warrants correction for residual CSA effects in measurements of pseudocontact shifts, J. Am. Chem. Soc. 127 (2005) 17190-17191.

[82] A. Canales, A. Mallagaray, M.A. Berbís, A. Navarro-Vázquez, G. Domínguez, F.J. Cañada, S. André, H.J. Gabius, J. Pérez-Castells, J. Jiménez-Barbero, Lanthanide-chelating carbohydrate conjugates are useful tools to characterize carbohydrate conformation in solution and sensitive sensors to detect carbohydrate-protein interactions, J. Am. Chem. Soc. 136 (2014) 8011-8017.

[83] L. Banci, I. Bertini, L.K. Bren, A.M. Cremonini, B.H. Gray, C. Luchinat, P. Turano, The use of pseudocontact shifts to refine solution structures of paramagnetic metalloproteins: Met80Ala cyano-cytochrome $c$ as an example, J. Biol. Inorg. Chem. 1 (1996) 117-126.

[84] L. Banci, I. Bertini, J.G. Huber, C. Luchinat, A. Rosato, Partial orientation of oxidized and reduced cytochrome $b_{5}$ at high magnetic fields: Magnetic susceptibility anisotropy contributions and consequences for protein solution structure determination, J. Am. Chem. Soc. 120 (1998) 12903-12909.

[85] M. Stanton-Cook, X.-C. Su, G. Otting, T. Huber, pyParaTools v0.8-alpha, in: http://zenodo.org/record/10313\#.V5GDL454yCg, 2011.

[86] M. Rinaldelli, A. Carlon, E. Ravera, G. Parigi, C. Luchinat, FANTEN: a new web-based interface for the analysis of magnetic anisotropy-induced NMR data, J. Biomol. NMR 61 (2015) 21-34.

[87] S.P. Skinner, M. Moshev, M.A. Hass, P.H. Keizers, M. Ubbink, PARAssignparamagnetic NMR assignments of protein nuclei on the basis of pseudocontact shifts, J. Biomol. NMR 55 (2013) 379-389.

[88] S.P. Skinner, M. Moshev, M.A.S. Hass, P.H.J. Keizers, M. Ubbink, Erratum to: PARAssign - paramagnetic NMR assignments of protein nuclei on the basis of pseudocontact shifts, J. Biomol. NMR 56 (2013) 401.

[89] M. Gochin, H. Roder, Protein structure refinement based on paramagnetic NMR shifts: Applications to wild-type and mutant forms of cytochrome $c$, Protein Sci. 4 (1995) 296-305.

[90] L. Banci, I. Bertini, G.G. Savellini, A. Romagnoli, P. Turano, M.A. Cremonini, C. Luchinat, H.B. Gray, Pseudocontact shifts as constraints for energy minimization and molecular dynamics calculations on solution structures of paramagnetic metalloproteins, Proteins 29 (1997) 68-76.

[91] J.C. Hus, D. Marion, M. Blackledge, De novo determination of protein structure by NMR using orientational and long-range order restraints, J. Mol. Biol. 298 (2000) 927-936. 
[92] P. Güntert, Automated NMR structure calculation with CYANA, Meth. Mol. Biol. 278 (2004) 353-378.

[93] C.D. Schwieters, J.J. Kuszewski, N. Tjandra, G.M. Clore, The Xplor-NIH NMR molecular structure determination package, J. Magn. Reson. 160 (2003) 65-73.

[94] C.D. Schwieters, J.J. Kuszewski, G. Marius Clore, Using Xplor-NIH for NMR molecular structure determination, Prog. NMR Spectrosc. 48 (2006) 47-62.

[95] G.N. Murshudov, P. Skubák, A.A. Lebedev, N.S. Pannu, R.A. Steiner, R.A. Nicholls, M.D. Winn, F. Long, A.A. Vagin, REFMAC5 for the refinement of macromolecular crystal structures, Acta Cryst. D 67 (2011) 355-367.

[96] M. Rinaldelli, E. Ravera, V. Calderone, G. Parigi, G.N. Murshudov, C. Luchinat, Simultaneous use of solution NMR and X-ray data in REFMAC5 for joint refinement/detection of structural differences, Acta Cryst. D 70 (2014) 958-967.

[97] Y. Shen, O. Lange, F. Delaglio, P. Rossi, J.M. Aramini, G. Liu, A. Eletsky, Y. Wu, K.K. Singarapu, A. Lemak, A. Ignatchenko, C.H. Arrowsmith, T. Szyperski, G.T. Montelione, D. Baker, A. Bax, Consistent blind protein structure generation from NMR chemical shift data, Proc. Natl. Acad. Sci. USA 105 (2008) 4685-4690.

[98] C. Schmitz, R. Vernon, G. Otting, D. Baker, T. Huber, Protein structure determination from pseudocontact shifts using ROSETTA, J. Mol. Biol. 416 (2012) 668-677.

[99] H. Yagi, K.B. Pilla, A. Maleckis, B. Graham, T. Huber, G. Otting, Three-dimensional protein fold determination from backbone amide pseudocontact shifts generated by lanthanide tags at multiple sites, Structure 21 (2013) 883-890.

[100] K.B. Pilla, G. Otting, T. Huber, Pseudocontact shift-driven iterative resampling for 3D structure determinations of large proteins, J. Mol. Biol. 428 (2016) 522-532.

[101] E.E. Snyder, B.W. Buoscio, J.J. Falke, Calcium(II) site specificity: effect of size and charge on metal ion binding to an EF-hand-like site, Biochemistry 29 (1990) 3937-3943.

[102] E. Pidcock, G.R. Moore, Structural characteristics of protein binding sites for calcium and lanthanide ions, J. Biol. Inorg. Chem. 6 (2001) 479-489.

[103] L. Lee, B.D. Sykes, Proton nuclear magnetic resonance determination of the sequential ytterbium replacement of calcium in carp parvalbumin, Biochemistry 20 (1981) 1156-1162.

[104] L. Lee, B.D. Sykes, Use of lanthanide-induced nuclear magnetic resonance shifts for determination of protein structure in solution: EF calcium binding site of carp parvalbumin, Biochemistry 22 (1983) 4366-4373.

[105] R.R. Biekofsky, F.W. Muskett, J.M. Schmidt, S.R. Martin, J.P. Browne, P.M. Bayley, J. Feeney, NMR approaches for monitoring domain orientations in calcium-binding proteins in solution using partial replacement of $\mathrm{Ca}^{2+}$ by $\mathrm{Tb}^{3+}$, FEBS Lett. 460 (1999) 519-526.

[106] I. Bertini, I. Gelis, N. Katsaros, C. Luchinat, A. Provenzani, Tuning the affinity for lanthanides of calcium binding proteins, Biochemistry 42 (2003) 8011-8021.

[107] I. Bertini, Y.K. Gupta, C. Luchinat, G. Parigi, M. Peana, L. Sgheri, J. Yuan, Paramagnetism-based NMR restraints provide maximum allowed probabilities for the different conformations of partially independent protein domains, J. Am. Chem. Soc. 129 (2007) 12786-12794.

[108] S. Balayssac, B. Jiménez, M. Piccioli, Assignment strategy for fast relaxing signals: complete aminoacid identification in thulium substituted calbindin $\mathrm{D}_{9 \mathrm{~K}}$, J. Biomol. NMR 34 (2006) 63-73.

[109] G. Pintacuda, A.Y. Park, M.A. Keniry, N.E. Dixon, G. Otting, Lanthanide labeling offers fast NMR approach to 3D structure determinations of protein-protein complexes, J. Am. Chem. Soc. 128 (2006) 3696-3702.

[110] M.W. Frey, S.T. Frey, W.D. Horrocks, Jr., B.F. Kaboord, S.J. Benkovic, Elucidation of the metal-binding properties of the Klenow fragment of Escherichia coli polymerase I and 
bacteriophage T4 DNA polymerase by lanthanide(III) luminescence spectroscopy, Chem. Biol. 3 (1996) 393-403.

[111] C.A. Brautigam, K. Aschheim, T.A. Steitz, Structural elucidation of the binding and inhibitory properties of lanthanide (III) ions at the 3'-5' exonucleolytic active site of the Klenow fragment, Chem. Biol. 6 (1999) 901-908.

[112] C. Ma, S.J. Opella, Lanthanide ions bind specifically to an added "EF-hand" and orient a membrane protein in micelles for solution NMR spectroscopy, J. Magn. Reson. 146 (2000) 381-384.

[113] K.J. Franz, M. Nitz, B. Imperiali, Lanthanide-binding tags as versatile protein coexpression probes, ChemBioChem 4 (2003) 265-271.

[114] M. Nitz, K.J. Franz, R.L. Maglathlin, B. Imperiali, A powerful combinatorial screen to identify high-affinity terbium(III)-binding peptides, ChemBioChem 4 (2003) 272-276.

[115] M. Nitz, M. Sherawat, K.J. Franz, E. Peisach, K.N. Allen, B. Imperiali, Structural origin of the high affinity of a chemically evolved lanthanide-binding peptide, Angew. Chem. Int. Ed. 43 (2004) 3682-3685.

[116] J. Wöhnert, K.J. Franz, M. Nitz, B. Imperiali, H. Schwalbe, Protein alignment by a coexpressed lanthanide-binding tag for the measurement of residual dipolar couplings, J. Am. Chem. Soc. 125 (2003) 13338-13339.

[117] L.J. Martin, M.J. Hähnke, M. Nitz, J. Wöhnert, N.R. Silvaggi, K.N. Allen, H. Schwalbe, B. Imperiali, Double-lanthanide-binding tags: design, photophysical properties, and NMR applications, J. Am. Chem. Soc. 129 (2007) 7106-7113.

[118] A.W. Barb, T.G. Ho, H. Flanagan-Steet, J.H. Prestegard, Lanthanide binding and IgG affinity construct: potential applications in solution NMR, MRI, and luminescence microscopy, Protein Sci. 21 (2012) 1456-1466.

[119] A.W. Barb, G.P. Subedi, An encodable lanthanide binding tag with reduced size and flexibility for measuring residual dipolar couplings and pseudocontact shifts in large proteins, J. Biomol. NMR 64 (2016) 75-85.

[120] D. Barthelmes, M. Gränz, K. Barthelmes, K.N. Allen, B. Imperiali, T. Prisner, H. Schwalbe, Encoded loop-lanthanide-binding tags for long-range distance measurements in proteins by NMR and EPR spectroscopy, J. Biomol. NMR 63 (2015) 275-282.

[121] K. Barthelmes, A.M. Reynolds, E. Peisach, H.R.A. Jonker, N.J. DeNunzio, K.N. Allen, B. Imperiali, H. Schwalbe, Engineering encodable lanthanide-binding tags into loop regions of proteins, J. Am. Chem. Soc. 133 (2011) 808-819.

[122] X.-C. Su, T. Huber, N.E. Dixon, G. Otting, Site-specific labelling of proteins with a rigid lanthanide-binding tag, ChemBioChem 7 (2006) 1599-1604.

[123] X.-C. Su, K. McAndrew, T. Huber, G. Otting, Lanthanide-binding peptides for NMR measurements of residual dipolar couplings and paramagnetic effects from multiple angles, J. Am. Chem. Soc. 130 (2008) 1681-1687.

[124] T. Saio, K. Ogura, M. Yokochi, Y. Kobashigawa, F. Inagaki, Two-point anchoring of a lanthanide-binding peptide to a target protein enhances the paramagnetic anisotropic effect, J. Biomol. NMR 44 (2009) 157-166.

[125] T. Saio, K. Ogura, K. Shimizu, M. Yokochi, T.R. Burke, Jr., F. Inagaki, An NMR strategy for fragment-based ligand screening utilizing a paramagnetic lanthanide probe, J. Biomol. NMR 51 (2011) 395-408.

[126] T. Saio, M. Yokochi, H. Kumeta, F. Inagaki, PCS-based structure determination of protein-protein complexes, J. Biomol. NMR 46 (2010) 271-280.

[127] M. Erdélyi, E. d'Auvergne, A. Navarro-Vázquez, A. Leonov, C. Griesinger, Dynamics of the glycosidic bond: conformational space of lactose, Chem. Eur. J. 17 (2011) 9368-9376. 
[128] A. Mallagaray, A. Canales, G. Domínguez, J. Jiménez-Barbero, J. Pérez-Castells, A rigid lanthanide binding tag for NMR structural analysis of carbohydrates, Chem. Commun. 47 (2011) 7179-7181.

[129] S. Yamamoto, T. Yamaguchi, M. Erdélyi, C. Griesinger, K. Kato, Paramagnetic lanthanide tagging for NMR conformational analyses of N-linked oligosaccharides, Chem. Eur. J. 17 (2011) 9280-9282.

[130] A. Canales, A. Mallagaray, J. Pérez-Castells, I. Boos, C. Unverzagt, S. André, H.J. Gabius, F.J. Canada, J. Jiménez-Barbero, Breaking pseudo-symmetry in multiantennary complex N-glycans using lanthanide-binding tags and NMR pseudo-contact shifts, Angew. Chem. Int. Ed. 52 (2013) 13789-13793.

[131] A. Mallagaray, G. Domínguez, T. Peters, J. Pérez-Castells, A rigid lanthanide binding tag to aid NMR studies of a $70 \mathrm{kDa}$ homodimeric coat protein of human norovirus, Chem. Commun. 52 (2016) 601-604.

[132] U. Brath, S.I. Swamy, A.X. Veiga, C.C. Tung, F. Van Petegem, M. Erdélyi, Paramagnetic ligand tagging to identify protein binding sites, J. Am. Chem. Soc. 137 (2015) 11391-11398.

[133] J. Kalia, R.T. Raines, Advances in bioconjugation, Curr. Org. Chem. 14 (2010) 138147.

[134] T. Ikegami, L. Verdier, P. Sakhaii, S. Grimme, B. Pescatore, K. Saxena, K.M. Fiebig, C. Griesinger, Novel techniques for weak alignment of proteins in solution using chemical tags coordinating lanthanide ions, J. Biomol. NMR 29 (2004) 339-349.

[135] G. Pintacuda, A. Moshref, A. Leonchiks, A. Sharipo, G. Otting, Site-specific labelling with a metal chelator for protein-structure refinement, J. Biomol. NMR 29 (2004) 351-361.

[136] V. Gaponenko, A.S. Altieri, J. Li, R.A. Byrd, Breaking symmetry in the structure determination of (large) symmetric protein dimers, J. Biomol. NMR 24 (2002) 143-148.

[137] A. Dvoretsky, V. Gaponenko, P.R. Rosevear, Derivation of structural restraints using a thiol-reactive chelator, FEBS Lett. 528 (2002) 189-192.

[138] D.E. Kamen, S.M. Cahill, M.E. Girvin, Multiple alignment of membrane proteins for measuring residual dipolar couplings using lanthanide ions bound to a small metal chelator, J. Am. Chem. Soc. 129 (2007) 1846-1847.

[139] A. Leonov, B. Voigt, F. Rodriguez-Castañeda, P. Sakhaii, C. Griesinger, Convenient synthesis of multifunctional EDTA-based chiral metal chelates substituted with an $S$ mesylcysteine, Chem. Eur. J. 11 (2005) 3342-3348.

[140] P. Haberz, F. Rodriguez-Castañeda, J. Junker, S. Becker, A. Leonov, C. Griesinger, Two new chiral EDTA-based metal chelates for weak alignment of proteins in solution, Org. Lett. 8 (2006) 1275-1278.

[141] M. Prudêncio, J. Rohovec, J.A. Peters, E. Tocheva, M.J. Boulanger, M.E. Murphy, H.J. Hupkes, W. Kosters, A. Impagliazzo, M. Ubbink, A caged lanthanide complex as a paramagnetic shift agent for protein NMR, Chem. Eur. J. 10 (2004) 3252-3260.

[142] F. Peters, M. Maestre-Martinez, A. Leonov, L. Kovačič, S. Becker, R. Boelens, C. Griesinger, Cys-Ph-TAHA: a lanthanide binding tag for RDC and PCS enhanced protein NMR, J. Biomol. NMR 51 (2011) 329-337.

[143] Z. Zhou, Z.R. Lu, Gadolinium-based contrast agents for magnetic resonance cancer imaging, Wiley Interdiscip. Rev. Nanomed. Nanobiotechnol. 5 (2013) 1-18.

[144] S. Aime, A. Barge, F. Benetollo, G. Bombieri, M. Botta, F. Uggeri, A novel compound in the lanthanide(III) DOTA series. X-ray crystal and molecular structure of the complex $\mathrm{Na}\left[\mathrm{La}\left(\right.\right.$ DOTA)La(HDOTA)] $\cdot 10 \mathrm{H}_{2} \mathrm{O}$, Inorg. Chem. 36 (1997) 4287-4289.

[145] S. Aime, M. Botta, G. Ermondi, NMR study of solution structures and dynamics of lanthanide(III) complexes of DOTA, Inorg. Chem. 31 (1992) 4291-4299. 
[146] S. Aime, A. Barge, M. Botta, M. Fasano, J. Danilo Ayala, G. Bombieri, Crystal structure and solution dynamics of the lutetium(III) chelate of DOTA, Inorg. Chim. Acta 246 (1996) 423-429.

[147] M.D. Vlasie, C. Comuzzi, A.M. van den Nieuwendijk, M. Prudêncio, M. Overhand, M. Ubbink, Long-range-distance NMR effects in a protein labeled with a lanthanide-DOTA chelate, Chem. Eur. J. 13 (2007) 1715-1723.

[148] P.H. Keizers, J.F. Desreux, M. Overhand, M. Ubbink, Increased paramagnetic effect of a lanthanide protein probe by two-point attachment, J. Am. Chem. Soc. 129 (2007) 92929293.

[149] M. Polášek, J. Kotek, P. Hermann, I. Císařová, K. Binnemans, I. Lukeš, Lanthanide(III) complexes of pyridine- $N$-oxide analogues of DOTA in solution and in the solid state. A new kind of isomerism in complexes of DOTA-like ligands, Inorg. Chem. 48 (2009) 466-475.

[150] M. Polášek, J. Rudovský, P. Hermann, I. Lukeš, L.V. Elst, R.N. Muller, Lanthanide(III) complexes of a pyridine $N$-oxide analogue of DOTA: exclusive $M$ isomer formation induced by a six-membered chelate ring, Chem. Commun. (2004) 2602-2603.

[151] M.A. Hass, P.H. Keizers, A. Blok, Y. Hiruma, M. Ubbink, Validation of a lanthanide tag for the analysis of protein dynamics by paramagnetic NMR spectroscopy, J. Am. Chem. Soc. 132 (2010) 9952-9953.

[152] M.A. Hass, W.M. Liu, R.V. Agafonov, R. Otten, L.A. Phung, J.T. Schilder, D. Kern, M. Ubbink, A minor conformation of a lanthanide tag on adenylate kinase characterized by paramagnetic relaxation dispersion NMR spectroscopy, J. Biomol. NMR 61 (2015) 123-136.

[153] W.M. Liu, P.H. Keizers, M.A. Hass, A. Blok, M. Timmer, A.J. Sarris, M. Overhand, M. Ubbink, A pH-sensitive, colorful, lanthanide-chelating paramagnetic NMR probe, J. Am. Chem. Soc. 134 (2012) 17306-17313.

[154] W.M. Liu, S.P. Skinner, M. Timmer, A. Blok, M.A. Hass, D.V. Filippov, M. Overhand, M. Ubbink, A two-armed lanthanoid-chelating paramagnetic NMR probe linked to proteins via thioether linkages, Chem. Eur. J. 20 (2014) 6256-6258.

[155] M.D. Lee, M.L. Dennis, J.D. Swarbrick, B. Graham, Enantiomeric two-armed lanthanide-binding tags for complementary effects in paramagnetic NMR spectroscopy, Chem. Commun. 52 (2016) 7954-7957.

[156] D. Häussinger, J.R. Huang, S. Grzesiek, DOTA-M8: An extremely rigid, high-affinity lanthanide chelating tag for PCS NMR spectroscopy, J. Am. Chem. Soc. 131 (2009) 1476114767.

[157] A.C. Opina, M. Strickland, Y.S. Lee, N. Tjandra, R.A. Byrd, R.E. Swenson, O. Vasalatiy, Analysis of the isomer ratios of polymethylated-DOTA complexes and the implications on protein structural studies, Dalton Trans. 45 (2016) 4673-4687.

[158] M. Strickland, C.D. Schwieters, C. Göbl, A.C.L. Opina, M.-P. Strub, R.E. Swenson, O. Vasalatiy, N. Tjandra, Characterizing the magnetic susceptibility tensor of lanthanidecontaining polymethylated-DOTA complexes, J. Biomol. NMR 66 (2016) 125-139.

[159] Y. Hikone, G. Hirai, M. Mishima, K. Inomata, T. Ikeya, S. Arai, M. Shirakawa, M. Sodeoka, Y. Ito, A new carbamidemethyl-linked lanthanoid chelating tag for PCS NMR spectroscopy of proteins in living HeLa cells, J. Biomol. NMR 66 (2016) 99-110.

[160] B. Graham, C.T. Loh, J.D. Swarbrick, P. Ung, J. Shin, H. Yagi, X. Jia, S. Chhabra, N. Barlow, G. Pintacuda, T. Huber, G. Otting, DOTA-amide lanthanide tag for reliable generation of pseudocontact shifts in protein NMR spectra, Bioconjugate Chem. 22 (2011) 2118-2125.

[161] L. de la Cruz, T.H. Nguyen, K. Ozawa, J. Shin, B. Graham, T. Huber, G. Otting, Binding of low molecular weight inhibitors promotes large conformational changes in the dengue virus NS2B-NS3 protease: fold analysis by pseudocontact shifts, J. Am. Chem. Soc. 133 (2011) 19205-19215. 
[162] M.D. Lee, C.T. Loh, J. Shin, S. Chhabra, M.L. Dennis, G. Otting, J.D. Swarbrick, B. Graham, Compact, hydrophilic, lanthanide-binding tags for paramagnetic NMR spectroscopy, Chem. Sci. 6 (2015) 2614-2624.

[163] E.H. Abdelkader, M.D. Lee, A. Feintuch, M.R. Cohen, J.D. Swarbrick, G. Otting, B. Graham, D. Goldfarb, A new $\mathrm{Gd}^{3+}$ spin label for $\mathrm{Gd}^{3+}-\mathrm{Gd}^{3+}$ distance measurements in proteins produces narrow distance distributions, J. Phys. Chem. Lett. 6 (2015) 5016-5021.

[164] F. Yang, X. Wang, B.B. Pan, X.-C. Su, Single-armed phenylsulfonated pyridine derivative of DOTA is rigid and stable paramagnetic tag in protein analysis, Chem. Commun. 52 (2016) 11535-11538.

[165] C.C. Liu, P.G. Schultz, Adding new chemistries to the genetic code, Annu. Rev. Biochem. 79 (2010) 413-444.

[166] C.T. Loh, K. Ozawa, K.L. Tuck, N. Barlow, T. Huber, G. Otting, B. Graham, Lanthanide tags for site-specific ligation to an unnatural amino acid and generation of pseudocontact shifts in proteins, Bioconjugate Chem. 24 (2013) 260-268.

[167] C.T. Loh, B. Graham, E.H. Abdelkader, K.L. Tuck, G. Otting, Generation of pseudocontact shifts in proteins with lanthanides using small "clickable" nitrilotriacetic acid and iminodiacetic acid tags, Chem. Eur. J. 21 (2015) 5084-5092.

[168] E.H. Abdelkader, A. Feintuch, X. Yao, L.A. Adams, L. Aurelio, B. Graham, D. Goldfarb, G. Otting, Protein conformation by EPR spectroscopy using gadolinium tags clicked to genetically encoded $p$-azido-L-phenylalanine, Chem. Commun. 51 (2015) 1589815901.

[169] I. Grenthe, Stability relationships among the rare earth dipicolinates, J. Am. Chem. Soc. 83 (1961) 360-364.

[170] X.-C. Su, B. Man, S. Beeren, H. Liang, S. Simonsen, C. Schmitz, T. Huber, B.A. Messerle, G. Otting, A dipicolinic acid tag for rigid lanthanide tagging of proteins and paramagnetic NMR spectroscopy, J. Am. Chem. Soc. 130 (2008) 10486-10487.

[171] B. Man, X.-C. Su, H. Liang, S. Simonsen, T. Huber, B.A. Messerle, G. Otting, 3Mercapto-2,6-pyridinedicarboxylic acid: a small lanthanide-binding tag for protein studies by NMR spectroscopy, Chem. Eur. J. 16 (2010) 3827-3832.

[172] X. Jia, A. Maleckis, T. Huber, G. Otting, 4,4'-Dithiobisdipicolinic acid: A small and convenient lanthanide binding tag for protein NMR spectroscopy, Chem. Eur. J. 17 (2011) 6830-6836.

[173] Q.F. Li, Y. Yang, A. Maleckis, G. Otting, X.-C. Su, Thiol-ene reaction: a versatile tool in site-specific labelling of proteins with chemically inert tags for paramagnetic NMR, Chem. Commun. 48 (2012) 2704-2706.

[174] Y. Yang, Q.F. Li, C. Cao, F. Huang, X.-C. Su, Site-specific labeling of proteins with a chemically stable, high-affinity tag for protein study, Chem. Eur. J. 19 (2013) 1097-1103.

[175] R.S. Ma, Q.F. Li, A.D. Wang, J.H. Zhang, Z.J. Liu, J.H. Wu, X.-C. Su, K. Ruan, Determination of pseudocontact shifts of low-populated excited states by NMR chemical exchange saturation transfer, Phys. Chem. Chem. Phys. 18 (2016) 13794-13798.

[176] F. Huang, Y.Y. Pei, H.H. Zuo, J.L. Chen, Y. Yang, X.-C. Su, Bioconjugation of proteins with a paramagnetic NMR and fluorescent tag, Chem. Eur. J. 19 (2013) 1714117149.

[177] J.-L. Chen, X. Wang, F. Yang, C. Cao, G. Otting, X.-C. Su, 3D structure determination of an unstable transient enzyme intermediate by paramagnetic NMR spectroscopy, Angew. Chem. Int. Ed. 55 (2016) 13744-13748.

[178] Y. Yang, J.T. Wang, Y.Y. Pei, X.-C. Su, Site-specific tagging proteins via a rigid, stable and short thiolether tether for paramagnetic spectroscopic analysis, Chem. Commun. 51 (2015) 2824-2827. 
[179] J.D. Swarbrick, P. Ung, X.-C. Su, A. Maleckis, S. Chhabra, T. Huber, G. Otting, B. Graham, Engineering of a bis-chelator motif into a protein $\alpha$-helix for rigid lanthanide binding and paramagnetic NMR spectroscopy, Chem. Commun. 47 (2011) 7368-7370.

[180] M. Sattler, S.W. Fesik, Resolving resonance overlap in the NMR spectra of proteins from differential lanthanide-induced shifts, J. Am. Chem. Soc. 119 (1997) 7885-7886.

[181] X.-C. Su, H. Liang, K.V. Loscha, G. Otting, $\left[\operatorname{Ln}(D P A)_{3}\right]^{3-}$ is a convenient paramagnetic shift reagent for protein NMR studies, J. Am. Chem. Soc. 131 (2009) 10352-10353.

[182] H. Yagi, K.V. Loscha, X.-C. Su, M. Stanton-Cook, T. Huber, G. Otting, Tunable paramagnetic relaxation enhancements by $\left[\mathrm{Gd}(\mathrm{DPA})_{3}\right]^{3-}$ for protein structure analysis, J. Biomol. NMR 47 (2010) 143-153.

[183] X. Jia, H. Yagi, X.-C. Su, M. Stanton-Cook, T. Huber, G. Otting, Engineering $\left[\operatorname{Ln}(\mathrm{DPA})_{3}\right]^{3-}$ binding sites in proteins: a widely applicable method for tagging proteins with lanthanide ions, J. Biomol. NMR 50 (2011) 411-420.

[184] Z. Wei, Y. Yang, Q.F. Li, F. Huang, H.H. Zuo, X.-C. Su, Noncovalent tagging proteins with paramagnetic lanthanide complexes for protein study, Chem. Eur. J. 19 (2013) 57585764.

[185] M. Nomura, T. Kobayashi, T. Kohno, K. Fujiwara, T. Tenno, M. Shirakawa, I. Ishizaki, K. Yamamoto, T. Matsuyama, M. Mishima, C. Kojima, Paramagnetic NMR study of $\mathrm{Cu}^{2+}$ IDA complex localization on a protein surface and its application to elucidate long distance information, FEBS Lett. 566 (2004) 157-161.

[186] J.P. Gallivan, D.A. Dougherty, Cation- $\pi$ interactions in structural biology, Proc. Natl. Acad. Sci. USA 96 (1999) 9459-9464.

[187] J. Feeny, B. Birdsall, A.F. Bradbury, R.R. Biekofsky, P.M. Bayley, Calmodulin tagging provides a general method of using lanthanide induced magnetic field orientation to observe residual dipolar couplings in proteins in solution, J. Biomol. NMR 21 (2001) 41-48.

[188] A.R. Camacho-Zarco, F. Munari, M. Wegstroth, W.M. Liu, M. Ubbink, S. Becker, M. Zweckstetter, Multiple paramagnetic effects through a tagged reporter protein, Angew. Chem. Int. Ed. 54 (2015) 336-339.

[189] A. Dumas, L. Lercher, C.D. Spicer, B.G. Davis, Designing logical codon reassignment - expanding the chemistry in biology, Chem. Sci. 6 (2015) 50-69.

[190] D.H. Jones, S.E. Cellitti, X. Hao, Q. Zhang, M. Jahnz, D. Summerer, P.G. Schultz, T. Uno, B.H. Geierstanger, Site-specific labeling of proteins with NMR-active unnatural amino acids, J. Biomol. NMR 46 (2010) 89-100.

[191] A.W. Foster, D. Osman, N.J. Robinson, Metal preferences and metallation, J. Biol. Chem. 289 (2014) 28095-28103.

[192] B.A. Krizek, D.L. Merkle, J.M. Berg, Ligand variation and metal ion binding specificity in zinc finger peptides, Inorg. Chem. 32 (1993) 937-940.

[193] M. Schmiedeskamp, R.E. Klevit, Paramagnetic cobalt as a probe of the orientation of an accessory DNA-binding region of the yeast ADR1 zinc-finger protein, Biochemistry 36 (1997) 14003-14011.

[194] L.V. Harper, B.T. Amann, V.K. Vinson, J.M. Berg, NMR studies of a cobalt-substituted zinc finger peptide, J. Am. Chem. Soc. 115 (1993) 2577-2580.

[195] A. Bavoso, A. Ostuni, G. Battistuzzi, L. Menabue, M. Saladini, M. Sola, Metal ion binding to a zinc finger peptide containing the Cys- $\mathrm{X}_{2}-\mathrm{Cys}-\mathrm{X}_{4}-\mathrm{His}_{-} \mathrm{X}_{4}-\mathrm{Cys}$ domain of a nucleic acid binding protein encoded by the Drosophila Fw-element, Biochem. Biophys. Res. Commun. 242 (1998) 385-389.

[196] L. Banci, L.B. Dugad, G.N. La Mar, K.A. Keating, C. Luchinat, R. Pierattelli, ${ }^{1}$ H nuclear magnetic resonance investigation of cobalt(II) substituted carbonic anhydrase, Biophys. J. 63 (1992) 530-543. 
[197] I. Bertini, B.H. Jonsson, C. Luchinat, R. Pierattelli, A.J. Vila, Strategies of signal assignments in paramagnetic metalloproteins. An NMR investigation of the thiocyanate adduct of the cobalt(II)-substituted human carbonic anhydrase II, J. Magn. Reson. B 104 (1994) 230-239.

[198] X. Wang, S. Srisailam, A.A. Yee, A. Lemak, C. Arrowsmith, J.H. Prestegard, F. Tian, Domain-domain motions in proteins from time-modulated pseudocontact shifts, J. Biomol. NMR 39 (2007) 53-61.

[199] S. Balayssac, I. Bertini, A. Bhaumik, M. Lelli, C. Luchinat, Paramagnetic shifts in solid-state NMR of proteins to elicit structural information, Proc. Natl. Acad. Sci. USA 105 (2008) 17284-17289.

[200] I. Bertini, A. Bhaumik, G. De Paëpe, R.G. Griffin, M. Lelli, J.R. Lewandowski, C. Luchinat, High-resolution solid-state NMR structure of a $17.6 \mathrm{kDa}$ protein, J. Am. Chem. Soc. 132 (2010) 1032-1040.

[201] I. Bertini, L. Emsley, M. Lelli, C. Luchinat, J. Mao, G. Pintacuda, Ultrafast MAS solidstate NMR permits extensive ${ }^{13} \mathrm{C}$ and ${ }^{1} \mathrm{H}$ detection in paramagnetic metalloproteins, J. Am. Chem. Soc. 132 (2010) 5558-5559.

[202] L. Benda, J. Mareš, E. Ravera, G. Parigi, C. Luchinat, M. Kaupp, J. Vaara, Pseudocontact NMR shifts over the paramagnetic metalloprotein CoMMP-12 from first principles, Angew. Chem. Int. Ed. (2016) 10.1002/anie.201608829.

[203] K. Tu, M. Gochin, Structure determination by restrained molecular dynamics using NMR pseudocontact shifts as experimentally determined constraints, J. Am. Chem. Soc. 121 (1999) 9276-9285.

[204] M. Gochin, Nuclear magnetic resonance studies of a paramagnetic metallo DNA complex, J. Am. Chem. Soc. 119 (1997) 3377-3378.

[205] M. Gochin, V. Khorosheva, M.A. Case, Structural characterization of a paramagnetic metal-ion-assembled three-stranded $\alpha$-helical coiled coil, J. Am. Chem. Soc. 124 (2002) 11018-11028.

[206] J. Swarbrick, P. Ung, M. Dennis, M. Lee, S. Chhabra, B. Graham, Installation of a rigid EDTA-like motif into a protein $\alpha$-helix for paramagnetic NMR spectroscopy with Co(II) ions, Chem. Eur. J. 22 (2015) 1228-1232.

[207] Y. Yang, F. Huang, T. Huber, X.-C. Su, Site-specific tagging proteins with a rigid, small and stable transition metal chelator, 8-hydroxyquinoline, for paramagnetic NMR analysis, J. Biomol. NMR 64 (2016) 103-113.

[208] V.V. Novikov, A.A. Pavlov, A.S. Belov, A.V. Vologzhanina, A. Savitsky, Y.Z. Voloshin, Transition ion strikes back: Large magnetic susceptibility anisotropy in cobalt(II) clathrochelates, J. Phys. Chem. Lett. 5 (2014) 3799-3803.

[209] T.H. Nguyen, K. Ozawa, M. Stanton-Cook, R. Barrow, T. Huber, G. Otting, Generation of pseudocontact shifts in protein NMR spectra with a genetically encoded cobalt(II)-binding amino acid, Angew. Chem. Int. Ed. 50 (2011) 692-694.

[210] S.H. Park, V.S. Wang, J. Radoicic, A.A. De Angelis, S. Berkamp, S.J. Opella, Paramagnetic relaxation enhancement of membrane proteins by incorporation of the metalchelating unnatural amino acid 2-amino-3-(8-hydroxyquinolin-3-yl)propanoic acid (HQA), J. Biomol. NMR 61 (2015) 185-196.

[211] V. Gaponenko, S. Sarma, A. Altieri, D. Horita, J. Li, R.A. Byrd, Improving the accuracy of NMR structures of large proteins using pseudocontact shifts as long-range restraints, J. Biomol. NMR 28 (2004) 205-212.

[212] I. Bertini, P. Kursula, C. Luchinat, G. Parigi, J. Vahokoski, M. Wilmanns, J. Yuan, Accurate solution structures of proteins from X-ray data and a minimal set of NMR data: calmodulin-peptide complexes as examples, J. Am. Chem. Soc. 131 (2009) 5134-5144. 
[213] J. Li, K.B. Pilla, Q. Li, Z. Zhang, X.-C. Su, T. Huber, J. Yang, Magic angle spinning NMR structure determination of proteins from pseudocontact shifts, J. Am. Chem. Soc. 135 (2013) 8294-8303.

[214] G. Kervern, S. Steuernagel, F. Engelke, G. Pintacuda, L. Emsley, Absence of Curie relaxation in paramagnetic solids yields long ${ }^{1} \mathrm{H}$ coherence lifetimes, J. Am. Chem. Soc. 129 (2007) 14118-14119.

[215] I. Bertini, V. Calderone, L. Cerofolini, M. Fragai, C.F. Geraldes, P. Hermann, C. Luchinat, G. Parigi, J.M. Teixeira, The catalytic domain of MMP-1 studied through tagged lanthanides, FEBS Lett. 586 (2012) 557-567.

[216] B. Qian, S. Raman, R. Das, P. Bradley, A.J. McCoy, R.J. Read, D. Baker, Highresolution structure prediction and the crystallographic phase problem, Nature 450 (2007) 259-264.

[217] E. Liepinsh, M. Baryshev, A. Sharipo, M. Ingelman-Sundberg, G. Otting, S. Mkrtchian, Thioredoxin fold as homodimerization module in the putative chaperone ERp29: NMR structures of the domains and experimental model of the $51 \mathrm{kDa}$ dimer, Structure 9 (2001) 457-471.

[218] D.J. Crick, J.X. Wang, B. Graham, J.D. Swarbrick, H.R. Mott, D. Nietlispach, Integral membrane protein structure determination using pseudocontact shifts, J. Biomol. NMR 61 (2015) 197-207.

[219] K.B. Pilla, J.K. Leman, G. Otting, T. Huber, Capturing conformational states in proteins using sparse paramagnetic NMR data, PLoS One 10 (2015) e0127053.

[220] T. Müntener, D. Häussinger, P. Selenko, F.X. Theillet, In-cell protein structures from 2D NMR experiments, J. Phys. Chem. Lett. 7 (2016) 2821-2825.

[221] B.B. Pan, F. Yang, Y. Ye, Q. Wu, C. Li, T. Huber, X.-C. Su, 3D structure determination of a protein in living cells using paramagnetic NMR spectroscopy, Chem. Commun. 52 (2016) 10237-10240.

[222] M. Ubbink, M. Ejdebäck, B.G. Karlsson, D.S. Bendall, The structure of the complex of plastocyanin and cytochrome $f$, determined by paramagnetic NMR and restrained rigid-body molecular dynamics, Structure 6 (1998) 323-335.

[223] R. Hulsker, M.V. Baranova, G.S. Bullerjahn, M. Ubbink, Dynamics in the transient complex of plastocyanin-cytochrome $f$ from Prochlorothrix hollandica, J. Am. Chem. Soc. 130 (2008) 1985-1991.

[224] P.H. Keizers, B. Mersinli, W. Reinle, J. Donauer, Y. Hiruma, F. Hannemann, M. Overhand, R. Bernhardt, M. Ubbink, A solution model of the complex formed by adrenodoxin and adrenodoxin reductase determined by paramagnetic NMR spectroscopy, Biochemistry 49 (2010) 6846-6855.

[225] Y. Kobashigawa, T. Saio, M. Ushio, M. Sekiguchi, M. Yokochi, K. Ogura, F. Inagaki, Convenient method for resolving degeneracies due to symmetry of the magnetic susceptibility tensor and its application to pseudo contact shift-based protein-protein complex structure determination, J. Biomol. NMR 53 (2012) 53-63.

[226] L. de la Cruz, W.N. Chen, B. Graham, G. Otting, Binding mode of the activitymodulating C-terminal segment of NS2B to NS3 in the dengue virus NS2B-NS3 protease, FEBS J. 281 (2014) 1517-1533.

[227] K. Ozawa, S. Jergic, A.Y. Park, N.E. Dixon, G. Otting, The proofreading exonuclease subunit $\varepsilon$ of Escherichia coli DNA polymerase III is tethered to the polymerase subunit $\alpha$ via a flexible linker, Nucleic Acids Res. 36 (2008) 5074-5082.

[228] D. Shishmarev, Y. Wang, C.E. Mason, X.-C. Su, A.J. Oakley, B. Graham, T. Huber, N.E. Dixon, G. Otting, Intramolecular binding mode of the C-terminus of Escherichia coli single-stranded DNA binding protein determined by nuclear magnetic resonance spectroscopy, Nucleic Acids Res. 42 (2014) 2750-2757. 
[229] C. Göbl, M. Resch, M. Strickland, C. Hartlmüller, M. Viertler, N. Tjandra, T. Madl, Increasing the chemical-shift dispersion of unstructured proteins with a covalent lanthanide shift reagent, Angew. Chem. Int. Ed. (2016) 10.1002/anie.201607261.

[230] O. Kratky, G. Porod, Röntgenuntersuchung gelöster Fadenmoleküle, Recl. Trav. Chim. Pays-Bas 68 (1949) 1106-1122.

[231] N.K. Williams, E. Liepinsh, S.J. Watt, P. Prosselkov, J.M. Matthews, P. Attard, J.L. Beck, N.E. Dixon, G. Otting, Stabilization of native protein fold by intein-mediated covalent cyclization, J. Mol. Biol. 346 (2005) 1095-1108.

[232] I. Bertini, C. Del Bianco, I. Gelis, N. Katsaros, C. Luchinat, G. Parigi, M. Peana, A. Provenzani, M.A. Zoroddu, Experimentally exploring the conformational space sampled by domain reorientation in calmodulin, Proc. Natl. Acad. Sci. USA 101 (2004) 6841-6846.

[233] I. Bertini, A. Giachetti, C. Luchinat, G. Parigi, M.V. Petoukhov, R. Pierattelli, E. Ravera, D.I. Svergun, Conformational space of flexible biological macromolecules from average data, J. Am. Chem. Soc. 132 (2010) 13553-13558.

[234] S. Dasgupta, X. Hu, P.H. Keizers, W.M. Liu, C. Luchinat, M. Nagulapalli, M. Overhand, G. Parigi, L. Sgheri, M. Ubbink, Narrowing the conformational space sampled by two-domain proteins with paramagnetic probes in both domains, J. Biomol. NMR 51 (2011) 253-263.

[235] C. Luchinat, M. Nagulapalli, G. Parigi, L. Sgheri, Maximum occurrence analysis of protein conformations for different distributions of paramagnetic metal ions within flexible two-domain proteins, J. Magn. Reson. 215 (2012) 85-93.

[236] W. Andrałojć, K. Berlin, D. Fushman, C. Luchinat, G. Parigi, E. Ravera, L. Sgheri, Information content of long-range NMR data for the characterization of conformational heterogeneity, J. Biomol. NMR 62 (2015) 353-371.

[237] C. Camilloni, M. Vendruscolo, Using pseudocontact shifts and residual dipolar couplings as exact NMR restraints for the determination of protein structural ensembles, Biochemistry 54 (2015) 7470-7476.

[238] L. Russo, M. Maestre-Martinez, S. Wolff, S. Becker, C. Griesinger, Interdomain dynamics explored by paramagnetic NMR, J. Am. Chem. Soc. 135 (2013) 17111-17120.

[239] E.H. Abdelkader, X. Yao, A. Feintuch, L.A. Adams, L. Aurelio, B. Graham, D. Goldfarb, G. Otting, Pulse EPR-enabled interpretation of scarce pseudocontact shifts induced by lanthanide binding tags, J. Biomol. NMR 64 (2016) 39-51.

[240] J.L. Chen, Y. Yang, L.L. Zhang, H. Liang, T. Huber, X.-C. Su, G. Otting, Analysis of the solution conformations of T4 lysozyme by paramagnetic NMR spectroscopy, Phys. Chem. Chem. Phys. 18 (2016) 5850-5859.

[241] L. Unione, G. Ortega, A. Mallagaray, F. Corzana, J. Pérez-Castells, A. Canales, J. Jiménez-Barbero, O. Millet, Unraveling the conformational landscape of ligand binding to glucose/galactose-binding protein by paramagnetic NMR and MD simulations, ACS Chem. Biol. 11 (2016) 2149-2157.

[242] S.P. Skinner, W.M. Liu, Y. Hiruma, M. Timmer, A. Blok, M.A. Hass, M. Ubbink, Delicate conformational balance of the redox enzyme cytochrome P450cam, Proc. Natl. Acad. Sci. USA 112 (2015) 9022-9027.

[243] T. Saio, K. Ogura, H. Kumeta, Y. Kobashigawa, K. Shimizu, M. Yokochi, K. Kodama, H. Yamaguchi, H. Tsujishita, F. Inagaki, Ligand-driven conformational changes of MurD visualized by paramagnetic NMR, Sci. Rep. 5 (2015) 16685.

[244] X. Xu, P.H. Keizers, W. Reinle, F. Hannemann, R. Bernhardt, M. Ubbink, Intermolecular dynamics studied by paramagnetic tagging, J. Biomol. NMR 43 (2009) 247254.

[245] Y. Hiruma, M.A. Hass, Y. Kikui, W.M. Liu, B. Ölmez, S.P. Skinner, A. Blok, A. Kloosterman, H. Koteishi, F. Löhr, H. Schwalbe, M. Nojiri, M. Ubbink, The structure of the 
cytochrome p450cam-putidaredoxin complex determined by paramagnetic NMR spectroscopy and crystallography, J. Mol. Biol. 425 (2013) 4353-4365.

[246] K.D. Brewer, T. Bacaj, A. Cavalli, C. Camilloni, J.D. Swarbrick, J. Liu, A. Zhou, P. Zhou, N. Barlow, J. Xu, A.B. Seven, E.A. Prinslow, R. Voleti, D. Häussinger, A.M. Bonvin, D.R. Tomchick, M. Vendruscolo, B. Graham, T.C. Südhof, J. Rizo, Dynamic binding mode of a Synaptotagmin-1-SNARE complex in solution, Nat. Struct. Mol. Biol. 22 (2015) 555564.

[247] C. Eichmüller, N.R. Skrynnikov, Observation of microsecond time-scale protein dynamics in the presence of $\mathrm{Ln}^{3+}$ ions: application to the $\mathrm{N}$-terminal domain of cardiac troponin C, J. Biomol. NMR 37 (2007) 79-95.

[248] L. Poppe, C.M. Tegley, V. Li, J. Lewis, J. Zondlo, E. Yang, R.J. Kurzeja, R. Syed, Different modes of inhibitor binding to prolyl hydroxylase by combined use of X-ray crystallography and NMR spectroscopy of paramagnetic complexes, J. Am. Chem. Soc. 131 (2009) 16654-16655.

[249] M. John, G. Pintacuda, A.Y. Park, N.E. Dixon, G. Otting, Structure determination of protein-ligand complexes by transferred paramagnetic shifts, J. Am. Chem. Soc. 128 (2006) 12910-12916.

[250] T. Zhuang, H.S. Lee, B. Imperiali, J.H. Prestegard, Structure determination of a Galectin-3-carbohydrate complex using paramagnetism-based NMR constraints, Protein Sci. 17 (2008) 1220-1231.

[251] K. Jaudzems, X. Jia, H. Yagi, D. Zhulenkovs, B. Graham, G. Otting, E. Liepinsh, Structural basis for 5'-end-specific recognition of single-stranded DNA by the R3H domain from human Subp-2, J. Mol. Biol. 424 (2012) 42-53.

[252] W.N. Chen, C. Nitsche, K.B. Pilla, B. Graham, T. Huber, C.D. Klein, G. Otting, Sensitive NMR approach for determining the binding mode of tightly binding ligand molecules to protein targets, J. Am. Chem. Soc. 138 (2016) 4539-4546.

[253] E. Balogh, D. Wu, G. Zhou, M. Gochin, NMR second site screening for structure determination of ligands bound in the hydrophobic pocket of HIV-1 gp41, J. Am. Chem. Soc. 131 (2009) 2821-2823.

[254] L. Skrisovska, M. Schubert, F.H. Allain, Recent advances in segmental isotope labeling of proteins: NMR applications to large proteins and glycoproteins, J. Biomol. NMR 46 (2010) 51-65.

[255] S.E. Cellitti, D.H. Jones, L. Lagpacan, X. Hao, Q. Zhang, H. Hu, S.M. Brittain, A. Brinker, J. Caldwell, B. Bursulaya, G. Spraggon, A. Brock, Y. Ryu, T. Uno, P.G. Schultz, B.H. Geierstanger, In vivo incorporation of unnatural amino acids to probe structure, dynamics, and ligand binding in a large protein by nuclear magnetic resonance spectroscopy, J. Am. Chem. Soc. 130 (2008) 9268-9281.

[256] T. Yabuki, T. Kigawa, N. Dohmae, K. Takio, T. Terada, Y. Ito, E.D. Laue, J.A. Cooper, M. Kainosho, S. Yokoyama, Dual amino acid-selective and site-directed stable-isotope labeling of the human c-Ha-Ras protein by cell-free synthesis, J. Biomol. NMR 11 (1998) 295-306.

[257] A. Feintuch, G. Otting, D. Goldfarb, $\mathrm{Gd}^{3+}$ spin labeling for measuring distances in biomacromolecules: Why and how? Methods Enzymol. 563 (2015) 415-457. 


\section{Glossary of abbreviations}

3MDPA: 3-mercaptodipicolinic acid

4MDPA: 4-mercaptodipicolinic acid

4MMDPA: 4-mercaptomethyldipicolinic acid

4MMPyMTA: 2,2',2",2"'-(((4-(mercaptomethyl)pyridine-2,6-

diyl)bis(methylene))bis(azanetriyl)tetraacetic acid

4MTDA: 4'-mercapto-2,2':6', $2^{\prime \prime}$-terpyridine-6, $6^{\prime \prime}$-dicarboxylic acid

4PS-DPA: 4-(phenylsulfonyl)dipicolinic acid

4PS-PyMTA: 4-phenylsulfonyl-(pyridin-2,6-diyl)bismethylenenitrilo tetrakis(acetic acid)

4VPyMTA: 4-vinyl(pyridine-2,6-diyl)bismethylenenitrilotetrakisacetic acid

$\operatorname{ArgN}$ : E. coli arginine repressor

BpyAla: (2,2'-bipyridin-5-yl)-L-alanine

BTTAA: 2-(4-((bis((1-(tert-butyl)-1H-1,2,3-triazol-4-yl)methyl)amino)methyl)-1H-1,2,3-

triazol-1-yl)acetic acid

CEST: chemical exchange saturation transfer

CLaNP: caged lanthanide NMR probe

CSA: chemical shift anisotropy

DFT: density functional theory

DOTA: 1,4,7,10-tetraazacyclododecane-1,4,7,10-tetraacetic acid

$D P A$ : dipicolinic acid

DTPA: diethylenetriaminepentaacetic acid

DTNB: 5,5'-dithiobis-2-nitrobenzoic acid

EDTA: ethylenediaminetetraacetic acid

HQ-Ala: 2-amino-3-(8-hydroxyquinolin-3-yl)propanoic acid

HSQC: heteronuclear single quantum coherence 
IDA: iminodiacetic acid

$L B P$ : lanthanide binding peptide

MTSL:

$S$-(1-oxyl-2,2,5,5-tetramethyl-2,5-dihydro-1H-pyrrol-3-yl)methyl

methanesulfonothioate

NOE: nuclear Overhauser effect

NTA: nitrilotriacetic acid

PCS: pseudocontact shift

PRE: paramagnetic relaxation enhancement

$R A C S$ : residual anisotropic chemical shift

$R D C$ : residual dipolar coupling

$R M S D$ : root mean square deviation

SAP: square antiprism

TAHA: triaminohexaacetate

TOCSY: total correlated spectroscopy

TSAP: twisted square antiprism

UTR: unique tensor representation 